\title{
The dynamics of production in advance : three essays in how pre-made decisions on inventory can influence revenue-maximization and the nature of competition
}

Citation for published version (APA):

van den Berg, A. H. J. (2012). The dynamics of production in advance : three essays in how pre-made decisions on inventory can influence revenue-maximization and the nature of competition. [Doctoral Thesis, Maastricht University]. Maastricht University. https://doi.org/10.26481/dis.20120120ab

Document status and date:

Published: 01/01/2012

DOI:

10.26481/dis.20120120ab

Document Version:

Publisher's PDF, also known as Version of record

Please check the document version of this publication:

- A submitted manuscript is the version of the article upon submission and before peer-review. There can be important differences between the submitted version and the official published version of record.

People interested in the research are advised to contact the author for the final version of the publication, or visit the DOI to the publisher's website.

- The final author version and the galley proof are versions of the publication after peer review.

- The final published version features the final layout of the paper including the volume, issue and page numbers.

Link to publication

\footnotetext{
General rights rights.

- You may freely distribute the URL identifying the publication in the public portal. please follow below link for the End User Agreement:

www.umlib.nl/taverne-license

Take down policy

If you believe that this document breaches copyright please contact us at:

repository@maastrichtuniversity.nl

providing details and we will investigate your claim.
}

Copyright and moral rights for the publications made accessible in the public portal are retained by the authors and/or other copyright owners and it is a condition of accessing publications that users recognise and abide by the legal requirements associated with these

- Users may download and print one copy of any publication from the public portal for the purpose of private study or research.

- You may not further distribute the material or use it for any profit-making activity or commercial gain

If the publication is distributed under the terms of Article 25fa of the Dutch Copyright Act, indicated by the "Taverne" license above, 


\section{The Dynamics of Production in Advance}

Three essays in how pre-made decisions on inventory can influence revenue-maximization and the nature of competition

Anita Helena Johanna van den Berg 
This book was typeset by the author using LaTeX.

All rights reserved. No part of this publication may be reproduced, stored in a retrieval system, or transmitted, in any form, or by any means, electronic, mechanical, photocopying, recording or otherwise, without the prior permission in writing from the author.

The Dynamics of Production in Advance: Three essays in how pre-made decisions on inventory can influence revenue-maximization and the nature of competition

(C) Anita Helena Johanna van den Berg, 2011

Cover illustration by Toàn Phan 


\section{The Dynamics of Production in Advance}

Three essays in how pre-made decisions on inventory can influence revenue-maximization and the nature of competition

Proefschrift

ter verkrijging van de graad van doctor aan de Universiteit Maastricht, op gezag van Rector Magnificus, Prof. mr. G.P.M.F. Mols, volgens het besluit van het College van Decanen, in het openbaar te verdedigen

op vrijdag 20 januari 2012 om 10.00 uur

door

Anita Helena Johanna van den Berg 


\section{Promotor:}

Prof. dr. H.J.M. Peters

\section{Copromotor:}

Prof. dr. P.J.J. Herings

\section{Beoordelingscommissie:}

Prof. dr. M.A. Carree (voorzitter)

Dr. R. Peeters

Prof. dr. M.A. Satterthwaite

Dit onderzoek werd financieel mogelijk gemaakt door Maastricht Research School of Economics of Technology and Organizations (METEOR). 


\section{Acknowledgments}

The last few years have been a period of continuous learning, academically, but also personally. One of the results of this learning experience you are holding in your hands right now: my Ph.D.-thesis. This thesis would not have looked the way it does today without the valuable input of many others whom I'd like to have the opportunity to thank here.

First of all, I'd like to thank my supervisors Hans Peters and JeanJacques Herings. Hans, the way in which you have guided me during my research is how in my opinion all supervisors should guide their Ph.D.'s. Figuratively speaking, you let me do all the walking and choose my own directions, but when I got lost, you were there to guide me back to the right track again. And, when there was a trunk blocking the direction I wanted to go in, I could always call on you for helping me lift it. Jean-Jacques, whenever I got your approval for my calculations, I could count on them being one hundred percent mathematically correct. You have taught me to be precise and never to take anything for granted, no matter how sound it may seem. You have the ability to push a person to the top of her capabilities. Mark Satterthwaite, thank you for giving me the opportunity to visit Kellogg and experience academia in the US. I very much enjoyed our conversations about my research, but also about health care in the US and other topics. It was a real delight to meet you, a devoted academic with such a wide field of interest.

Academic support only is not always sufficient to stay focused on ones research. Having a great group of people around me helped me a lot. I'd like to thank my colleagues and fellow Ph.D.-students of the Department of Quantitative Economics for creating a relaxed working environment. Irem, 
thank you for the countless coffee breaks, the always entertaining conversations and your support. And of course, I'd like to thank Haydee and Karin, for making it easy for me not to get distracted by any of the administrative stuff.

My evenings in Maastricht were usually filled with activities. Every Monday night, there were the great moments and new moves that I shared with the women at bellydance school Salomé. Thank you for your always positive attitude, ladies! Many of the other nights I spent at Bodyvision, where the owners and members succeeded in creating an atmosphere that often felt more like a cousy living room than a gym.

Also outside of Maastricht, there are a lot of people whose support and care mean a great deal to me. Even though I didn't see you as often as I'd liked to, I knew and know that I can always count on you: my friends from high school (Eefje, Eveline, Jeske, Karlijn, Martje, Suzanne and Willeke) and Joyce. My time in the USA was not only academically a very interesting experience. It gave me the opportunity to meet a lot of different people and spend many great evenings with them. With some of you, Toàn, Didi, Matthias and Amina, I have even spent some amazing (and slightly crazy) holidays in Europe with. Hopefully, these holidays will turn on out to be a recurring event. I'd also like to thank my parents, Nico and Petra, who taught me to be independent and follow my heart.

And then there is that one person, that I could never overlook. My coauthor, my best friend, my continuous support and the one with whom I intend to (finally) share an apartment with in the old folks' home. Thank you, Iwan, for making my life so much richer this past decade. 


\section{Contents}

1 Introduction $\quad 9$

2 Dynamic Duopoly with Intertemporal Capacity Constraints 15

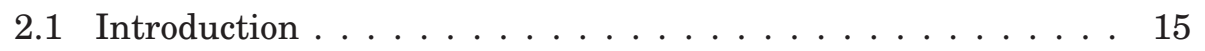

2.2 Model . . . . . . . . . . . . . . . . . . . . . . . 18

2.3 Commitment . . . . . . . . . . . . . . . 19

2.4 Non-commitment . . . . . . . . . . . . . . . . . 26

2.5 Commitment versus Non-commitment . . . . . . . . . . . 39

2.6 Concluding Remarks . . . . . . . . . . . . . . . . . . . . . . 44

2.A The Reduced Best Response Correspondence . . . . . . . . . 46

2.B Subgame Perfect Equilibria . . . . . . . . . . . . . 50

3 The Impact of Bulk-Supply on a Dealer's Sales Strategy 67

3.1 Introduction . . . . . . . . . . . . . . . 67

3.2 The model . . . . . . . . . . . . . . . . . 69

3.3 The control problem . . . . . . . . . . . . . . 70

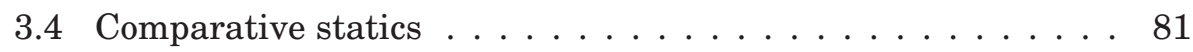

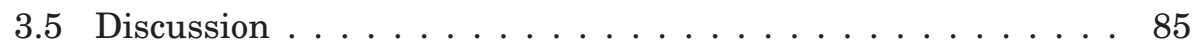

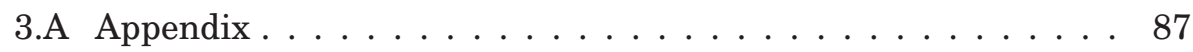

4 Collusion in a Price-Quantity Oligopoly 97

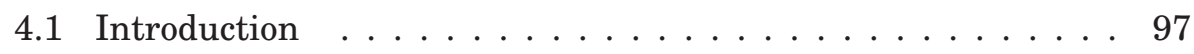

4.2 Model . . . . . . . . . . . . . . . . . . . . . . . . . . . 102

4.3 Static Nash Equilibrium . . . . . . . . . . . . . 103

4.4 Cartel Problem . . . . . . . . . . . . . . . . . . . 105 
4.5 Collusion with Production in Advance . . . . . . . . . . . 108

4.5.1 Price Cartel . . . . . . . . . . . . . . . . . . . . . 108

4.5.2 Price-Quota Cartel . . . . . . . . . . . . . . . . . . 110

4.6 Production to Order . . . . . . . . . . . . . . . . . . 112

4.6.1 Static Nash Equilibrium . . . . . . . . . . . . . . 113

4.6 .2 Collusion . . . . . . . . . . . . . . . . . . . 113

4.7 Discussion . . . . . . . . . . . . . . . . . . . . . . . . . 114

4.8 Concluding Remarks . . . . . . . . . . . . . . . . . . 117

4. A Appendix . . . . . . . . . . . . . . . . . . 118

$\begin{array}{lr}\text { Bibliography } & 129\end{array}$

Nederlandse Samenvatting $\quad 135$

$\begin{array}{ll}\text { Curriculum Vitae } & 139\end{array}$ 


\section{Chapter 1}

\section{Introduction}

In this thesis, several markets are analyzed in which firms are not able to produce to order; instead, they can only sell what they already have in stock. The reasons for a firm not to be able to produce to order can be numerous. The most common one is that a firm does not produce the good itself, but buys it from a third-party, and the delivery of a new order takes time. Or the firm does produce its own goods, but it takes time to start up the machines and produce a new batch. Producing/purchasing products in advance limits the quantity output choices of a firm (and its possible competitors), which has an effect on the revenue, prices and competition-level - if multiple firms supply the market.

That the extent to which firms are flexible in their price and output decisions has a big effect on the market outcome, has been a topic of interest of economists for many years. Already in 1838, Auguste Cournot described two firms competing in the sales of a homogeneous good. In the model, taking its competitor's output as given, a firm chooses its quantity output to maximize profits. The price for which the good is eventually sold is the market clearing price. It turns out that in Cournot's model, both firms make a strictly positive profit. Joseph Bertrand, in 1883, criticized this model. He claimed that, since the firms sell exactly the same product, the only thing that is important for consumers is the price of this product. The firm that sells it for the lowest price shall accordingly get all of the market demand. Therefore, in his model, the firms compete in price, not in quantity. Given that its competitor prices above its marginal costs, a firm will slightly undercut this price and thereby serve the whole market. Since this holds for both the firms, it turns out that in the end, both firms will set a price that 
is exactly equal to marginal costs and make zero profit (given that marginal costs are constant and equal for both firms). Both the models and their results, Cournot's as well as Bertrand's, can easily be extended from duopoly to oligopoly.

The difference in firm profits between the models has stirred up the discussion about which of the two is the more accurate one - do firms compete in quantity or in price? This question is not easily answered and depends very much on the market where the competition takes place. If quantity output decisions cannot be changed in the short-run, the extra demand that a firm gains from undercutting its competitors in price, will go to waste. On the other hand, if a firm can easily serve the extra demand gained from cutting in price, it will be appealing to do so. In sum, how easily a firm can adjust or renew the quantity of the product it is selling, will have a big influence on its profits.

In some markets, firms may only to some extent be flexible in their quantity decisions in the short-run. Kreps and Scheinkman (1983) described a duopoly in which firms first have to invest in capacity, after which they compete in price. Under certain assumptions, they find that in their model price competition actually leads to Cournot outcomes, thereby bridging the gap between Bertrand and Cournot's models. In other settings, flexibility in quantity setting is restricted by assuming that firms have to deal with a given capacity constraint. Up to the constraint, they can easily adjust their output, but supplying more than their capacity limit is infinitely costly. In most models analyzed in the literature, the firms all face the same capacity constraint. The analysis increases quickly in complexity if firms face individual capacity constraints. Therefore, individual capacity constraints are usually used only in models with at most two firms. If there is no firm for whom it holds that its competitor(s) together can supply the whole market demand when price is equal to marginal costs, all firms expect a strictly positive profit when competing in price. This is shown in a simple model by Levitan and Shubik (1972), and has been extended by Allen and Hellwig (1993) and Osborne and Pitchik (1986).

Another method that has been used to overcome the differences in outcomes between the two models, is to find an intermediate model. In the last two decades, a strand of literature has appeared in which some of the firms compete in price and others in quantity. ${ }^{1}$ The common factor in all

\footnotetext{
${ }^{1}$ Examples of articles in which a simultaneous price and quantity competition is analyzed in a homogeneous framework are Allen (1992) and Qin and Stuart (1997).
} 
the models described in the last three paragraphs is that less flexibility in output decisions leads to less severe competition and therefore an increase in profits.

Flexibility in quantity decisions does not only effect market outcomes in competition, but also has an effect on the opportunities to collude between firms. Collusion is not possible if the firms only meet for one single period. The reason for that is that a firm that defects in that period, cannot be punished for it by its competitors afterwards. Ergo, every firm has an incentive to defect. However, this changes when market contact takes place an infinite amount of times - or, as it can be interpreted in practice, the firms have no idea when market contact will come to an end. If future profits are valuable enough and firms are not limited in their choice of capacity, it is a well known result that any price between the competitive price and the monopoly price can be colluded on. This is because the gain in profit from defecting one period cannot make up for the future losses caused by the breakdown of collusion. However, if a firm faces a restrictive capacity constraint, defecting from the collusive price is less profitable, since it cannot supply the whole market demand for the lower price it defects with. On the other hand, if its competitors together do not have enough capacity to serve the market at the competitive price, the future losses caused by the breakdown of collusion are also less severe. Both these effects influence the likeliness that collusion will actually take place. If competitors collude explicitly on one or several of their strategic variables, this agreement is also called a cartel.

When analyzing the effect of quantity limitations, the market structure cannot be neglected. A monopolist needs only to consider how its own output choices today will influence its set of possibilities tomorrow, the day after tomorrow, etcetera. It does not gain any extra profit from a less severe competition, since there is none. Firms that operate on a duopolistic or oligopolistic market need to consider that as well, but take into account at the same time how their current output choices will influence the future output choices of their competitors.

A fixed per-period capacity constraint is not the only way in which firms can be limited in their quantity flexibility. In some models, like in Benoit and Krishna (1987), firms can invest in capacity accumulation. Or there is one pool from which all firms extract their resource, as is sometimes the case with exhaustible resources. In the following, it is described how the firms in this thesis are restricted in their quantity flexibility and what the consequences of these limitations are. 
Chapter 2 deals with a duopoly, in which the firms have a time-span of two periods in which they compete in the sales of their exogenously given individual inventory. After those two periods, their product is worthless. In making their quantity output decisions, the firms have to take into account not only how this will affect their own inventory level and current profit, but also future competition. In the next chapter, no longer a fixed end-period is given and ordering new inventory is now also part of the possibilities. The analysis is restricted to a monopolistic market structure. The goal of the monopolist is to determine the best moment to order a new batch and to maximize its profit on sales. In the last chapter of this thesis, in an oligopolistic setting, firms make an individual price and quantity decision in every period. Two types of production are considered: production in advance and production to order. For both types of production, it is analyzed under which conditions collusion between firms is sustainable and which form of collusion is preferred: collusion in price only, or collusion in price ánd quantity.

\section{Dynamic Duopoly with Intertemporal Capacity Con- straints}

In this chapter, a duopoly is studied in which both firms start with an exogenously given capacity, which they cannot renew. They both know that the demand for the product they are selling will only last for two periods, after that, their stock is worthless. Examples for these type of markets are, for instance, fashion items. The price that they will receive for their products each period is the market clearing price. We distinguish two cases. In the first, both firms already decide on their sales quantity for each period before competition actually takes place. We will call this commitment. It is shown that in this case, there exists a unique pure equilibrium in which, among others, price goes up over time. And if the difference in capacity between the two firms increases, consumer surplus and total surplus decrease. In the other case that is described, firms decide on their second period sales after the first period of competition already has taken place. We will call this noncommitment. It is found that here a pure equilibrium does not always exist. And if an equilibrium does exist, it depends on the starting capacities of the firms whether price will actually go up or down over time. 


\section{The Impact of Bulk-Supply on a Dealer's Sales Strat- egy}

A firm/dealer with monopolistic power is described in this chapter. The dealer in question can only renew its supply by large batches. Reasons for this are either because it can only buy the finished product from a wholesaler or an important part of production is only delivered in large bulks. We analyze the best moment to reorder and the optimal sales strategy between those reordering moments. It is found that the dealer always reorders after the same amount of time. Right after receiving the new batch, its sales quantity is the highest and this quantity continuously decreases until its new batch arrives. Its sales pattern is always the same in between any two consecutive, ordering moments. If future profits become less valuable, contrary to what one might think beforehand, there are settings in which the dealer will increase the time before it reorders.

\section{Collusion in a Price-Quantity Oligopoly}

This chapter studies an oligopoly in which the firms have both price and quantity as strategic variables. The game has an infinite time horizon, and the strategic variables can be adjusted every period. Two types of industry are analyzed, production in advance and production to order. In competition, for both modes of production, the expected profit of all firms is zero. To increase their profits, the firms can collude in price only, or in both strategic variables. It is found that, when production is in advance of sales and market demand is relatively inelastic, collusion in price only may not be sustainable and an additional agreement on outputs may be required to overcome coordination and incentive problems. On top of this, a price-quota cartel is more profitable than a price cartel. With production to order, collusion may not be sustainable without an agreement on production levels. 



\section{Chapter 2}

\section{Dynamic Duopoly with Intertemporal Capacity Constraints}

\subsection{Introduction}

In most models of dynamic duopoly, it is assumed that production is instantly adjusted to per-period demand. However, in many real-world applications, this is not the case. Take for instance a market vendor. He buys his inventory at a wholesaler in batches. Until the next batch arrives, he has to decide every day how much of his inventory he is willing to sell at that day's market. To maximize profits, the vendor has to take into account how selling part of his inventory today influences the profits he can make on the remainder of its stock. Moreover, he will have to take into account how its actions today will affect the behavior of its competitors the following days.

This chapter analyzes quantity competition in situations where production precedes sales and sales take place during a number of periods. ${ }^{1}$ As a result, the firm operates under a multi-period capacity constraint. Any production process that involves batch production would fit this description. Other examples concern settings with costly transportation, causing stores to be supplied only every few periods. Another relevant case can be found in the field of exhaustible resources. Firms at the source cannot renew their supply, but have many periods to sell the resource.

\footnotetext{
${ }^{1}$ This chapter is based on the paper van den Berg et al. (2011a).
} 
We address a number of questions related to the dynamics of the market structure, the development of prices and sales over time, and the implications for profits and consumer surplus. We examine the simplest situation possible: production or resource extraction has already taken place, the commodity is sold during two periods and demand is linear. Firms thereby effectively face a two-period capacity constraint.

In such a multi-period setting, it becomes relevant whether or not firms use current period outcomes before deciding upon their next period actions. We refer to these two possibilities as non-commitment versus commitment. In this chapter, both the non-commitment and the commitment case are analyzed and related to one another. In the commitment setting, the strategy of a firm specifies the amount it is going to supply at each period. This amount does not depend on the observed sales of the competing firm in the previous periods. This type of commitment is encountered, for instance, in situations where information about previous period(s) profits/sales is not processed before current period strategy decisions have to be made. In the non-commitment setting, the strategy of a firm describes how much stock to sell in each period, conditional on observed sales in previous periods by the competing firm. In the literature the commitment setting is often analyzed due to its better tractability. Nevertheless, in many settings, the non-commitment case is more realistic. We will show that the level of commitment can have a serious influence on the results.

In the commitment case, firms base their plan of action only on the level of initial stock of both the firms. In the exhaustible resource literature, this case has been analyzed for numerous settings similar to ours. This literature starts with Hotelling (1931). More recently, Loury (1986), Gaudet and Long (1994) and Lewis and Schmalensee (1980), all find results that are compatible with the results we find for the commitment setting. Wirl (2010) analyses a model with dynamics on the demand and supply side in which firms use a noncompetitive quantity strategy. We establish the existence of a unique Nash equilibrium. It is shown that, in this equilibrium, price increases over time and as a consequence, aggregate sales decrease over time. Aggregate sales per period depend on the distribution of initial production over the firms. Also, the firm with more stock will never leave the market before the smaller one does.

In the setting without commitment, a firm's supply is conditional on the amounts sold in the previous period. This makes it possible to adjust the sales path over time in response to observed sales by the competitor. In the 
non-commitment setting, there are some combinations of stock for which a subgame perfect Nash equilibrium does not exist. However, for most combinations, an equilibrium exists and is unique. In equilibrium, the firm with the larger initial production amount will never leave the market before the smaller firm. Equilibria in the non-commitment setting may exhibit counterintuitive features. For instance, price may decrease over time and therefore aggregate sales may increase over time. In the exhaustible resource literature, Salo and Tahvonen (2001) also analyze a non-commitment setting. Reinganum and Stokey (1985) analyze a setting in which firms extract their resource from a common property source and in which they can commit to certain extraction levels only for a limited number of periods.

We find that the equilibrium outcomes in the commitment-setting are equivalent to the equilibrium outcomes of the non-commitment setting for most combinations of initial stock. The outcomes differ when one of the firms is large and the other one is intermediate in stock size. In that situation, the large firm prefers not being able to commit, whereas the smaller firms prefers both firms committing. Consumer surplus is almost always higher in the commitment setting, except for some specific situations in which firms are very patient. Total surplus is at least as high in the commitment setting as it is in the non-commitment setting.

Apart from the literature on exhaustible resources, this chapter is related to those papers that analyze models with capacity constraints. Most of these papers, for instance Levitan and Shubik (1972), Osborne and Pitchik (1986) and Bikhchandani and Mamer (1993), use a setting in which firms compete in price. Bikhchandani and Mamer show that in their case of unit demand, there is a unique equilibrium, which is robust to extension over multiple periods and extension over additional sellers. Kreps and Scheinkman (1983) show in a dynamic game how an individually chosen capacity constraint in the first period can influence second-period price competition. More recently, several papers were written in which firms compete in quantity and are constrained in capacity. In Gabszewicz and Poddar (1997), firms choose their level of capacity before demand is known. After true consumers' demand is known, they compete in quantity for one period. It is shown that a symmetric subgame perfect Nash equilibrium exists. Laye and Laye (2008) analyze multi-market Cournot competition with capacity constraints. All firms can produce a limited amount of a homogeneous product. For this product they have to choose which part they will sell at every market. In this situation, a unique Cournot-Nash equilibrium exists. Besanko and Do- 
raszelski (2004) analyze a dynamic repeated setting in which firms in every period can invest in capacity and either compete in price or quantity. When firms compete in quantity, this leads to equal sized firms. However, when firms compete in price, over time the industry evolves towards asymmetric firm sizes.

To the best of our knowledge, the only other paper in the literature that uses an intertemporal capacity constraint, is Biglaiser and Vettas (2004). In their model, the two competing firms have an equal finite amount of product that they can sell in two periods. Demand is in units and growing, and firms compete in prices. The total demand over the two periods is more than one firm can produce, but less than both firms can produce together. An important feature of their model is that not only the sellers, but also the buyers act strategically. One of the results is that, when there is only one consumer, linear pricing implies there is no pure strategy equilibrium. Another paper that shows some resemblance with ours is the two-period model of Saloner (1987). In that paper, there are two periods of production, after which the goods are sold for the market clearing price. Saloner finds a unique subgame perfect equilibrium. The results of this paper have been extended by Pal $(1991,1996)$, in which the first of the papers allows for different costs in the two periods of production and the second one extends this model even further to allow for mixed strategies. Kovenock and Roy (1998) show that the work of Saloner is not robust against replacing the third stage by one of price competition, instead of assuming market clearing.

The chapter is organized as follows. The next section introduces the model. Section 2.3 analyzes the equilibria that result in the commitment case. The non-commitment situation is addressed in Section 2.4. In Section 2.5 , we analyze how the equilibrium outcomes in the commitment setting relate to the equilibrium outcomes in the non-commitment setting. Section 2.6 concludes. Lengthy and technical proofs are relegated to the appendix.

\subsection{Model}

We consider two profit maximizing firms that have produced (or bought) a homogeneous good. Firm $i=1,2$ therefore owns a finite amount $S_{i} \geq 0$ of the good. Since the goods are produced beforehand, the production costs are sunk and they do not play a role in the model. With their fixed amount of stock as an upperbound, the firms compete in quantity for two periods. A firm may choose to have residual supply at the end of the second period. The 
quantities sold by firm $i$ in period 1 and period 2 are denoted by $q_{i}$ and $r_{i}$, respectively, so $q_{i}+r_{i} \leq S_{i}$. The inverse demand each period is

$$
P(Q)=1-Q
$$

where $Q=q_{1}+q_{2}$ in the first period and $Q=r_{1}+r_{2}$ in the second. ${ }^{2,3}$ Profits earned in period 2 are discounted with a factor $\delta \in(0,1]$.

Two cases are analyzed. In the first, firms can commit to a sales strategy that is independent of sales by their competitor. That is, after production has taken place, both firms unconditionally decide how much they are going to sell in each period. This implies that firm $i$ 's strategy space is of the form $\Gamma_{i}=\left\{\left(q_{i}, r_{i}\right) \in \mathbb{R}_{+}^{2} \mid q_{i}+r_{i} \leq S_{i}\right\}$.

The second case is the one of non-commitment. In this case, the amount a firm is going to offer for sale in a period depends on the realized sales of its competitor in the previous period. As a result, the second-period strategy of a firm is now the specification of a sales quantity conditional on the observation of first-period sales. We define $F_{i}=\left\{f_{i}:\left[0, S_{1}\right] \times\left[0, S_{2}\right] \rightarrow\left[0, S_{i}\right] \mid\right.$ $\left.q_{i}+f_{i}\left(q_{1}, q_{2}\right) \leq S_{i}\right\}$ as the set of functions that assign a feasible second-period sales quantity to every possible combination of first-period sales. Firm $i$ 's strategy space is $\Sigma_{i}=\left[0, S_{i}\right] \times F_{i}$.

\subsection{Commitment}

In the commitment case firms choose a sales path that does not depend on their competitor's realized sales. Given strategies $\left(q_{1}, r_{1}\right) \in \Gamma_{1}$ and $\left(q_{2}, r_{2}\right) \in$ $\Gamma_{2}$, the profit $\Pi_{i}\left(q_{1}, r_{1}, q_{2}, r_{2}\right)$ of firm $i$ is given by

$$
\Pi_{i}\left(q_{1}, r_{1}, q_{2}, r_{2}\right)=q_{i} P\left(q_{i}+q_{j}\right)+\delta r_{i} P\left(r_{i}+r_{j}\right)
$$

When choosing its sales path $\left(q_{i}, r_{i}\right)$, firm $i$ takes the sales path $\left(q_{j}, r_{j}\right)$ of firm $j$ as given, where we use the notation $i$ and $j$ for the two competing firms. Firm $i$ therefore solves the problem

$$
\max _{q_{i}, r_{i}} \Pi_{i}\left(q_{1}, r_{1}, q_{2}, r_{2}\right)
$$

\footnotetext{
${ }^{2}$ The results in this chapter can be extended to the more general inverse demand functions of the form $P(Q)=a-b Q$, where $a, b>0$ and with firms facing unit costs of $c$, to be interpreted for instance as handling costs.

${ }^{3}$ We choose to analyze linear demand only to keep the analysis tractable.
} 
subject to

$$
q_{i}, r_{i} \geq 0 \text { and } q_{i}+r_{i} \leq S_{i} .
$$

The result is a best response $\gamma_{i}\left(q_{j}, r_{j}\right) \in \Gamma_{i}$ given by

$$
\gamma_{i}\left(q_{j}, r_{j}\right)=\left\{\begin{array}{cl}
\left(S_{i}, 0\right) & \left\{\begin{array}{l}
\text { if }\left[q_{j}-\delta r_{j}<1-\delta-2 S_{i},\right. \\
\left.q_{j}+r_{j} \leq 2-2 S_{i} \text { and } q_{j}, r_{j} \leq 1\right] \\
\text { or }\left[q_{j} \leq 1-2 S_{i} \text { and } r_{j}>1\right]
\end{array}\right. \\
\left(0, S_{i}\right) & \left\{\begin{array}{l}
\text { if }\left[q_{j}-\delta r_{j}>1-\delta+2 \delta S_{i},\right. \\
\left.q_{j}+r_{j} \leq 2-2 S_{i} \text { and } q_{j}, r_{j} \leq 1\right] \\
\text { or }\left[q_{j}>1 \text { and } r_{j} \leq 1-2 S_{i}\right]
\end{array}\right. \\
\left(\frac{1-\delta+2 \delta S_{i}-q_{j}+\delta r_{j}}{2+2 \delta}, \frac{2 S_{i}-1+\delta+q_{j}-\delta r_{j}}{2+2 \delta}\right) & \left\{\begin{array}{c}
\text { if } 1-\delta-2 S_{i} \leq q_{j}-\delta r_{j} \leq \\
1-\delta+2 \delta S_{i}, \\
q_{j}+r_{j} \leq 2-2 S_{i} \text { and } q_{j}, r_{j} \leq 1
\end{array}\right. \\
\left(\frac{1}{2}-\frac{1}{2} q_{j}, \frac{1}{2}-\frac{1}{2} r_{j}\right) & \left\{\begin{array}{c}
\text { if } q_{j}+r_{j}>2-2 S_{i} \\
\text { and } q_{j}, r_{j} \leq 1
\end{array}\right. \\
\left(0, \frac{1}{2}-\frac{1}{2} q_{j}, 0\right) & \left\{\begin{array}{c}
\text { if } 1-2 S_{i}<q_{j} \leq 1 \\
\text { and } r_{j}>1
\end{array}\right. \\
(0,0) & \left\{\begin{array}{c}
\text { if } 1-2 S_{i}<r_{j} \leq 1 \\
\text { and } q_{j}>1
\end{array}\right. \\
\text { if } q_{j}, r_{j}>1 .
\end{array}\right.
$$

The seven cases for $S_{i}$ are mutually exclusive and the best responses against $\left(q_{j}, r_{j}\right)$ are unique. The function $\gamma_{i}$ is continuous.

A pair of strategies $\left(q_{1}^{*}, r_{1}^{*}, q_{2}^{*}, r_{2}^{*}\right)$ is a Nash equilibrium iff

$$
\begin{aligned}
& \Pi_{1}\left(q_{1}^{*}, r_{1}^{*}, q_{2}^{*}, r_{2}^{*}\right) \geq \Pi_{1}\left(q_{1}, r_{1}, q_{2}^{*}, r_{2}^{*}\right) \text { for all }\left(q_{1}, r_{1}\right) \in \Gamma_{1}, \\
& \Pi_{2}\left(q_{1}^{*}, r_{1}^{*}, q_{2}^{*}, r_{2}^{*}\right) \geq \Pi_{2}\left(q_{1}^{*}, r_{1}^{*}, q_{2}, r_{2}\right) \text { for all }\left(q_{2}, r_{2}\right) \in \Gamma_{2},
\end{aligned}
$$

or, equivalently, $\gamma_{1}\left(q_{2}^{*}, r_{2}^{*}\right)=\left(q_{1}^{*}, r_{1}^{*}\right)$ and $\gamma_{2}\left(q_{1}^{*}, r_{1}^{*}\right)=\left(q_{2}^{*}, r_{2}^{*}\right)$.

Given any initial combination $\left(S_{1}, S_{2}, \delta\right)$, there is a unique equilibrium, as specified in Table 2.1 and depicted in Figure 2.1. In the figure, $\delta$ is fixed and $S_{1}, S_{2}$ are variable. A change of $\delta$ will not change the shape of the equilibrium areas, only the ratio between them. We use the superscript ' $c$ ' to refer to equilibria in the commitment case. The two letters in the subscript represent the relative level of stock of respectively firm $i$ and $j$, where 1 stands for low, $\mathrm{m}$ for medium and $\mathrm{h}$ for high. In Figure 2.1, also the number of active firms in each period is indicated, where $N_{1} / N_{2} / N_{r}$ represents 
respectively the number of firms that have strictly positive sales in the first period, the number of firms that have strictly positive sales in the second period, and the number of firms that have residual supply at the end of the second period. The figure shows that the number of active firms increases when production increases.

\begin{tabular}{|l|l|l|l|}
\hline & Parameter conditions & Period 1 & Period 2 \\
\hline$\left(\mathrm{X}_{\mathrm{ll}}^{c}\right)$ & $0 \leq S_{1}<\frac{1}{2}-\frac{1}{2} S_{2}-\frac{1}{2} \delta$ & $q_{1}^{c}=S_{1}$ & $r_{1}^{c}=0$ \\
& $0 \leq S_{2}<\frac{1}{2}-\frac{1}{2} S_{1}-\frac{1}{2} \delta$ & $q_{2}^{c}=S_{2}$ & $r_{2}^{c}=0$ \\
\hline$\left(\mathrm{X}_{\mathrm{lm}}^{c}\right)$ & $0 \leq S_{1}<\frac{1}{3}-\frac{1}{3} \delta$ & $q_{1}^{c}=S_{1}$ & $r_{1}^{c}=0$ \\
& $\frac{1}{2}-\frac{1}{2} \delta-\frac{1}{2} S_{1} \leq S_{2} \leq 1-\frac{1}{2} S_{1}$ & $q_{2}^{c}=\frac{1-S_{1}-\delta+2 \delta S_{2}}{2+2 \delta}$ & $r_{2}^{c}=\frac{2 S_{2}+S_{1}-1+\delta}{2+2 \delta}$ \\
\hline$\left(\mathrm{X}_{\mathrm{ml}}^{c}\right)$ & $\frac{1}{2}-\frac{1}{2} \delta-\frac{1}{2} S_{2} \leq S_{1} \leq 1-\frac{1}{2} S_{2}$ & $q_{1}^{c}=\frac{1-\delta+2 \delta S_{1}-S_{2}}{2+2 \delta}$ & $r_{1}^{c}=\frac{2 S_{1}+S_{2}-1+\delta}{2+2 \delta}$ \\
& $0 \leq S_{2}<\frac{1}{3}-\frac{1}{3} \delta$ & $q_{2}^{c}=S_{2}$ & $r_{2}^{c}=0$ \\
\hline$\left(\mathrm{X}_{\mathrm{lh}}^{c}\right)$ & $0 \leq S_{1}<\frac{1}{3}-\frac{1}{3} \delta$ & $q_{1}^{c}=S_{1}$ & $r_{1}^{c}=0$ \\
& $1-\frac{1}{2} S_{1}<S_{2}$ & $q_{2}^{c}=\frac{1}{2}-\frac{1}{2} S_{1}$ & $r_{2}^{c}=\frac{1}{2}$ \\
\hline$\left(\mathrm{X}_{\mathrm{hl}}^{c}\right)$ & $1-\frac{1}{2} S_{2}<S_{1}$ & $q_{1}^{c}=\frac{1}{2}-\frac{1}{2} S_{2}$ & $r_{1}^{c}=\frac{1}{2}$ \\
& $0 \leq S_{2}<\frac{1}{3}-\frac{1}{3} \delta$ & $q_{2}^{c}=S_{2}$ & $r_{2}^{c}=0$ \\
\hline$\left(\mathrm{X}_{\mathrm{mm}}^{c}\right)$ & $\frac{1}{3}-\frac{1}{3} \delta \leq S_{1} \leq 1-\frac{1}{2} S_{2}$ & $q_{1}^{c}=\frac{1-\delta+3 \delta S_{1}}{3+3 \delta}$ & $r_{1}^{c}=\frac{3 S_{1}-1+\delta}{3+3 \delta}$ \\
& $\frac{1}{3}-\frac{1}{3} \delta \leq S_{2} \leq 1-\frac{1}{2} S_{1}$ & $q_{2}^{c}=\frac{1-\delta+3 \delta S_{2}}{3+3 \delta}$ & $r_{2}^{c}=\frac{3 S_{2}-1+\delta}{3+3 \delta}$ \\
\hline$\left(\mathrm{X}_{\mathrm{mh}}^{c}\right)$ & $\frac{1}{3}-\frac{1}{3} \delta \leq S_{1} \leq \frac{2}{3}$ & $q_{1}^{c}=\frac{1-\delta+3 \delta S_{1}}{3+3+3 \delta}$ & $r_{1}^{c}=\frac{3 S_{1}-1+\delta}{3+3 \delta}$ \\
& $1-\frac{1}{2} S_{1}<S_{2}$ & $q_{2}^{c}=\frac{2+4 \delta-3 \delta S_{1}}{6+6 \delta}$ & $r_{2}^{c}=\frac{4+2 \delta-3 S_{1}}{6+6 \delta}$ \\
\hline$\left(\mathrm{X}_{\mathrm{hm}}^{c}\right)$ & $1-\frac{1}{2} S_{2}<S_{1}$ & $q_{1}^{c}=\frac{2+4 \delta-3 \delta S_{2}}{6+6 \delta}$ & $r_{1}^{c}=\frac{4+2 \delta-3 S_{2}}{6-6+6 \delta}$ \\
& $\frac{1}{3}-\frac{1}{3} \delta<S_{2} \leq \frac{2}{3}$ & $q_{2}^{c}=\frac{1-\delta+3 \delta S_{2}}{3+3 \delta}$ & $r_{2}^{c}=\frac{3 S_{2}-1+\delta}{3+3 \delta}$ \\
\hline$\left(\mathrm{X}_{\mathrm{hh}}^{c}\right)$ & $\frac{2}{3}<S_{1}$ & $q_{1}^{c}=\frac{1}{3}$ & $r_{1}^{c}=\frac{1}{3}$ \\
& $\frac{2}{3}<S_{2}$ & $q_{2}^{c}=\frac{1}{3}$ & $r_{2}^{c}=\frac{1}{3}$ \\
\hline
\end{tabular}

Table 2.1: Equilibria in the commitment case.

When the stock of firm 1 is low, as it is in Regions $\mathrm{X}_{\mathrm{ll}}^{\mathrm{c}}, \mathrm{X}_{\mathrm{lm}}^{\mathrm{c}}$, and $\mathrm{X}_{\mathrm{lh}}^{\mathrm{c}}$, it will sell all of its stock in the first period. These regions are non-empty only if the discount rate is strictly below one. The discounting of second-period profits gives firms an incentive to sell in period 1 rather than in period 2. When firm 1 has a low stock $S_{1}$, then selling this entirely in the first period will hardly decrease the marginal revenue in the first period. Consequently, as long as $\delta$ is not too high, marginal revenue in the second period will be less than the marginal revenue in the first period and firm 1 will sell its entire production in the first period. 


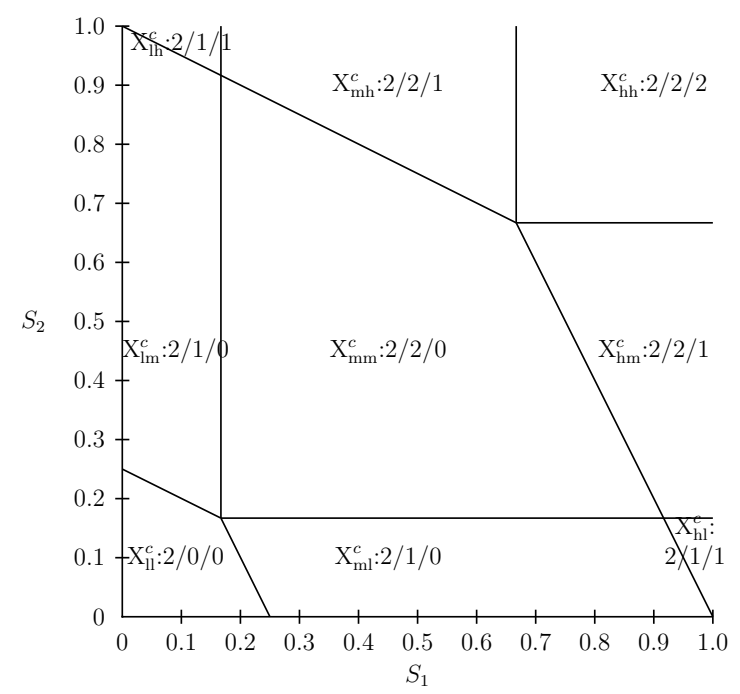

Figure 2.1: The commitment case: Equilibrium outcome regions and the number of firms that have stock in respectively period 1 , period 2 and after period 2, for $\delta=0.5$.

In Regions $\mathrm{X}_{\mathrm{ml}}^{\mathrm{c}}, \mathrm{X}_{\mathrm{mm}}^{\mathrm{c}}$, and $\mathrm{X}_{\mathrm{mh}}^{\mathrm{c}}$, firm 1 has an intermediate amount of the commodity in stock. It then maximizes profit by dividing its sales over the two periods in such a way that marginal revenue in both periods is equal.

In the remaining Regions, $\mathrm{X}_{\mathrm{hl}}^{\mathrm{c}}, \mathrm{X}_{\mathrm{hm}}^{\mathrm{c}}$ and $\mathrm{X}_{\mathrm{hh}}^{\mathrm{c}}$, firm 1 has a high stock and acts as if it has no capacity constraints. Firm 1 maximizes its profit in each period separately as to maximize total profit. It will have residual stock at the end of period 2.

A similar line of argumentation applies to the equilibrium strategy of firm 2. Note that in both periods in situation $\mathrm{X}_{\mathrm{hh}}^{\mathrm{c}}$ firms maximize their profit as if there is no capacity limit. This results in both firms choosing their Cournot equilibrium quantities of $\frac{1}{3}$ in both periods.

The next five propositions describe some comparative statics results for the case with commitment.

Proposition 2.3.1. In equilibrium, price weakly increases over time.

Proof For any given combination of $S_{1}, S_{2}$, and $\delta$, one can verify directly that $q_{1}^{\mathrm{c}}+q_{2}^{\mathrm{c}} \geq r_{1}^{\mathrm{c}}+r_{2}^{\mathrm{c}}$. Thus, the aggregate sales in the first period weakly exceed the aggregate sales in the second period and therefore price in the first period is less than or equal to the price in the second period. 
Notice, in particular, that as long as its capacity doesn't prevent it from doing so, a firm will adjust its sales to achieve equal marginal revenues in both periods. This together with a discount rate which is less than or equal to one implies that price cannot decrease over time.

Also the following proposition describes an intuitive result.

Proposition 2.3.2. An increase in $S_{i}$ leads to a weak increase in firm i's equilibrium profit.

Proof The derivative of the equilibrium profit function of firm $i$ with respect to $S_{i}$ is non-negative in every equilibrium outcome region and the profit function is continuous for all $\delta, S_{i}, S_{j} \geq 0$.

Notice, of course, that the profits in Proposition 2.3.2 correspond to sales revenues and do not take into account the costs of production.

The next proposition studies how the relative stock sizes of the two firms affect the commodity price. For fixed aggregate stock size $S_{1}+S_{2}$, we analyze how an increase in asymmetry $\left|S_{1}-S_{2}\right|$ influences equilibrium outcomes.

Proposition 2.3.3. Given fixed aggregate production $S_{1}+S_{2}$, an increase in $\left|S_{1}-S_{2}\right|$ leads to a weak decrease of first-period aggregate equilibrium sales and therefore a weak increase of first-period equilibrium price. It leads (i) to a decrease of second-period aggregate equilibrium sales and an increase of second-period equilibrium price in Regions $\mathrm{X}_{m h}^{\mathrm{c}}$ and $\mathrm{X}_{h m}^{\mathrm{c}}$ and (ii) to an increase of second-period aggregate equilibrium sales and a decrease of secondperiod equilibrium price in Regions $\mathrm{X}_{l m}^{\mathrm{c}}$ and $\mathrm{X}_{m l}^{\mathrm{c}}$. It has no effect on secondperiod aggregate equilibrium sales and equilibrium price in the other regions.

Proof Let $S=S_{1}+S_{2}$ be fixed and assume without loss of generality that $S_{2} \geq S_{1}$. Then $\left|S_{1}-S_{2}\right|$ increases if $S_{1}$ decreases. Since $S_{2} \geq S_{1}$, it holds that $\left(S_{1}, S_{2}, \delta\right) \in \mathrm{X}_{\mathrm{ll}}^{\mathrm{c}} \cup \mathrm{X}_{\mathrm{lm}}^{\mathrm{c}} \cup \mathrm{X}_{\mathrm{lh}}^{\mathrm{c}} \cup \mathrm{X}_{\mathrm{mm}}^{\mathrm{c}} \cup \mathrm{X}_{\mathrm{mh}}^{\mathrm{c}} \cup \mathrm{X}_{\mathrm{hh}}^{\mathrm{c}}$. Let $Q_{\mathrm{ab}}$ be the aggregate sales in equilibrium region $\mathrm{X}_{\mathrm{ab}}$.

For first-period aggregate sales, we find that

$$
\begin{array}{ll}
Q_{\mathrm{ll}}^{\mathrm{c}}=S, & \frac{\partial Q_{\mathrm{ll}}^{\mathrm{c}}}{\partial S_{1}}=0, \\
Q_{\mathrm{lm}}^{\mathrm{c}}=\frac{1-\delta+S_{1}+2 \delta S}{2+2 \delta}, & \frac{\partial Q_{\mathrm{lm}}^{\mathrm{c}}}{\partial S_{1}}=\frac{1}{2+2 \delta}>0, \\
Q_{\mathrm{lh}}^{\mathrm{c}}=\frac{1}{2}+\frac{1}{2} S_{1}, & \frac{\partial Q_{\mathrm{lh}}}{\partial S_{1}}=\frac{1}{2}>0, \\
Q_{\mathrm{mm}}^{\mathrm{c}}=\frac{2-2 \delta+3 \delta S}{3+3 \delta}, & \frac{\partial Q_{\mathrm{mm}}^{\mathrm{c}}}{\partial S_{1}}=0, \\
Q_{\mathrm{mh}}^{\mathrm{c}}=\frac{4+2 \delta+3 \delta S_{1}}{6+6 \delta}, & \frac{\partial Q_{\mathrm{mh}}^{\mathrm{c}}}{\partial S_{1}}=\frac{3 \delta}{6+6 \delta}>0, \\
Q_{\mathrm{hh}}^{\mathrm{c}}=\frac{2}{3}, & \frac{\partial Q_{\mathrm{hh}}^{1}}{\partial S_{1}}=0 .
\end{array}
$$


For second-period aggregate sales we have that

$$
\begin{array}{ll}
Q_{\mathrm{ll}}^{\mathrm{c}}=0, & \frac{\partial Q_{\mathrm{ll}}^{\mathrm{c}}}{\partial S_{1}}=0, \\
Q_{\mathrm{lm}}^{\mathrm{c}}=\frac{2 S-S_{1}-1+\delta}{2+2 \delta}, & \frac{\partial Q_{\mathrm{lm}}^{\mathrm{c}}}{\partial S_{1}}=-\frac{1}{2+2 \delta}<0, \\
Q_{\mathrm{lh}}^{\mathrm{c}}=\frac{1}{2}, & \frac{\partial Q_{\mathrm{lh}}^{\mathrm{c}}}{\partial S_{1}}=0, \\
Q_{\mathrm{mm}}^{\mathrm{c}}=\frac{3 S-2+2 \delta}{3+3 \delta}, & \frac{\partial Q_{\mathrm{mm}}^{\mathrm{c}}}{\partial S_{1}}=0, \\
Q_{\mathrm{mh}}^{\mathrm{c}}=\frac{3 S_{1}+2+4 \delta}{6+6 \delta}, & \frac{\partial Q_{\mathrm{mh}}^{\mathrm{c}}}{\partial S_{1}}=\frac{3}{6+6 \delta}>0, \\
Q_{\mathrm{hh}}^{\mathrm{c}}=\frac{2}{3}, & \frac{\partial Q_{\mathrm{hh}}^{1}}{\partial S_{1}}=0 .
\end{array}
$$

A larger difference in stocks results in a higher first-period price. This is intuitive: consider the extreme case where one of the firms is a monopolist, resulting in the highest possible first-period price. Surprisingly, the effect of increasing difference between the firms' stocks on second-period prices is ambiguous. In particular, it leads to a weak decrease in second-period price in Regions $\mathrm{X}_{\mathrm{lm}}^{\mathrm{c}}$ and $\mathrm{X}_{\mathrm{ml}}^{\mathrm{c}}$. In these regions, the smaller firm has no stock left at the beginning of period 2. An increase in the size of the bigger firm then simply leads to more sales by this firm in period 2 .

The following proposition studies the consequences of increased stocks for consumer surplus. Consumer surplus in the first period and in the second period is respectively $\frac{1}{2}\left(q_{1}+q_{2}\right)^{2}$ and $\frac{1}{2}\left(r_{1}+r_{2}\right)^{2}$. To compute the total consumer surplus we have to discount the second-period consumer surplus by $\delta$. Consumer surplus is therefore given by $\frac{1}{2}\left(q_{1}+q_{2}\right)^{2}+\frac{1}{2} \delta\left(r_{1}+r_{2}\right)^{2}$.

Proposition 2.3.4. Equilibrium consumer surplus weakly increases if the stock of at least one of the firms increases.

Proof It follows directly from the equilibrium outcomes that per-period sales weakly increase in $S_{1}$ and $S_{2}$.

Since the effect of an increase in $\left|S_{1}-S_{2}\right|$ on second-period sales is ambiguous by Proposition 2.3.3, it is not a priori clear how such an increase affects consumer surplus. The next proposition states, nevertheless, that this effect is unambiguously negative.

Proposition 2.3.5. Given fixed aggregate stock $S_{1}+S_{2}$, an increase in $\mid S_{1}-$ $S_{2}$ l leads to a weak decrease in equilibrium consumer surplus. 
Proof Proposition 2.3.3 implies a weak decrease in sales in both periods when $\left|S_{1}-S_{2}\right|$ increases, and therefore a weak decrease in consumer surplus, except possibly in Regions $\mathrm{X}_{\mathrm{lm}}^{\mathrm{c}}$ and $\mathrm{X}_{\mathrm{ml}}^{\mathrm{c}}$.

Consider some $\left(S_{1}, S_{2}, \delta\right)$ in Region $\mathrm{X}_{\mathrm{lm}}^{\mathrm{c}}$ or $\mathrm{X}_{\mathrm{ml}}^{\mathrm{c}}$. Assume without loss of generality that $S_{2} \geq S_{1}$, so $\left|S_{1}-S_{2}\right|$ increases if $S_{1}$ decreases. Then $\left(S_{1}, S_{2}, \delta\right)$ belongs to Region $\mathrm{X}_{\mathrm{lm}}^{\mathrm{c}}$. Consumer surplus is given by

$$
\frac{1}{2}\left(\frac{1-\delta+S_{1}+2 \delta S}{2+2 \delta}\right)^{2}-\frac{1}{2} \delta\left(\frac{2 S-S_{1}-1+\delta}{2+2 \delta}\right)^{2},
$$

where, as before, $S=S_{1}+S_{2}$. The derivative of the expression above with respect to $S_{1}$ is given by

$$
\frac{1-\delta+S_{1}+2 \delta S}{(2+2 \delta)^{2}}+\delta \frac{2 S-S_{1}-1+\delta}{(2+2 \delta)^{2}},
$$

which is easily shown to be non-negative.

An increase in inequality in firm sizes makes the bigger firm relatively more powerful compared to the smaller firm. This gives the bigger firm the opportunity to portray more "monopolist-like" behavior, which influences consumer surplus negatively.

By the same type of analysis, it can be shown that the results we have found for equilibrium consumer surplus coincide with the results that can be found for equilibrium total surplus. Total surplus is defined as the sum of consumer surplus and both the firms' surplus. In this case, total surplus is

$$
\left(q_{1}+q_{2}\right)\left(1-\frac{1}{2}\left(q_{1}+q_{2}\right)\right)+\delta\left(r_{1}+r_{2}\right)\left(1-\frac{1}{2}\left(r_{1}+r_{2}\right)\right) .
$$

Equilibrium total surplus weakly increases if production by at least one of the firms increases and, given fixed aggregate production $S_{1}+S_{2}$, an increase in $\left|S_{1}-S_{2}\right|$ leads to a weak decrease in equilibrium total surplus.

Summary of comparative statics results for the commitment case We find that, when firms have the power to commit to an unconditional sales strategy, price never decreases over time. A firm's profit increases when its stock increases and so does consumer surplus and total surplus. Finally, an increase in the difference between the stocks of the firms leads to lower sales in period 1 and lower consumer surplus and total surplus. The effect on period 2 sales is ambiguous. 


\subsection{Non-commitment}

We now study the case where the sales strategy of a firm in period 2 depends on the observed first-period sales. Once firms arrive in the second period of the game, they play a one-period game with capacity constraints. We analyze the subgame perfect Nash equilibria of the game. We do this by first analyzing the Nash equilibria of all possible period 2 subgames.

Consider the subgame $q=\left(q_{1}, q_{2}\right)$ in period 2 that results from firstperiod sales $\left(q_{1}, q_{2}\right)$ by the firms. Denote firm $i$ 's second-period stock by $T_{i}=S_{i}-q_{i}$. Now we can define $\sigma_{i q}:\left[0, T_{j}\right] \rightarrow\left[0, T_{i}\right]$ as firm $i$ 's best response function in subgame $q$. Given sales $r_{j}$ by firm $j$, firm $i$ solves the problem

$$
\max _{r_{i}} r_{i} P\left(r_{i}+r_{j}\right)
$$

subject to

$$
0 \leq r_{i} \leq T_{i} .
$$

The best response for firm $i$ in period 2 is then given by

$$
\sigma_{i q}\left(r_{j}\right)= \begin{cases}T_{i}, & \text { if } 0 \leq T_{i} \leq \frac{1}{2}-\frac{1}{2} r_{j}, \\ \max \left\{0, \frac{1}{2}-\frac{1}{2} r_{j}\right\}, & \text { otherwise. }\end{cases}
$$

Quantities $\left(r_{1}^{*}, r_{2}^{*}\right)$ are a Nash equilibrium of the second-period subgame $q$ if and only if $\sigma_{1 q}\left(r_{2}^{*}\right)=r_{1}^{*}$ and $\sigma_{2 q}\left(r_{1}^{*}\right)=r_{2}^{*}$. Each subgame $q$ has a unique Nash equilibrium as specified in Table 2.2 and depicted in Figure 2.2. ${ }^{4}$

In Region $\mathrm{Y}_{\mathrm{hh}}$, both firms have sufficient residual stock in the second period to choose their unconstrained profit maximizing sales quantity. In Regions $\mathrm{Y}_{\mathrm{hl}}$ and $\mathrm{Y}_{\mathrm{lh}}$, only one firm is restricted by its residual stock, respectively firm 1 and firm 2 . In Region $\mathrm{Y}_{11}$ both firms are restricted by their residual stock and sell in the second period all they have left.

The equilibrium action chosen by firm $i$ in period 2 is given by the function $f_{i}^{*}$ defined by

$$
f_{i}^{*}\left(q_{i}, q_{j}\right)= \begin{cases}\frac{1}{3}, & \text { if } T_{i}, T_{j}>\frac{1}{3}, \\ \frac{1}{2}-\frac{1}{2} T_{j}, & \text { if } T_{i}>\frac{1}{2}-\frac{1}{2} T_{j} \text { and } T_{j} \leq \frac{1}{3}, \\ T_{i}, & \text { if } T_{i} \leq \frac{1}{3} \text { or } T_{j} \leq 1-2 T_{i} .\end{cases}
$$

\footnotetext{
${ }^{4}$ This figure was earlier portrayed in Krishnan and Röller (1993), Figure 1. Their Stage 5 problem coincides with our second-period subgame.
} 


\begin{tabular}{c||c|c||c|c|c|c} 
& $T_{i}$ & $T_{j}$ & $r_{i}$ & $r_{j}$ & profits $i$ & profits $j$ \\
\hline \hline$\left(\mathrm{Y}_{\mathrm{hh}}\right)$ & $>\frac{1}{3}$ & $>\frac{1}{3}$ & $\frac{1}{3}$ & $\frac{1}{3}$ & $\frac{1}{9}$ & $\frac{1}{9}$ \\
\hline$\left(\mathrm{Y}_{\mathrm{lh}}\right)$ & $\leq \frac{1}{3}$ & $>\frac{1-T_{i}}{2}$ & $T_{i}$ & $\frac{1-T_{i}}{2}$ & $T_{i}\left(\frac{1-T_{i}}{2}\right)$ & $\left(\frac{1-T_{i}}{2}\right)^{2}$ \\
\hline$\left(\mathrm{Y}_{\mathrm{hl}}\right)$ & $>\frac{1-T_{j}}{2}$ & $\leq \frac{1}{3}$ & $\frac{1-T_{j}}{2}$ & $T_{j}$ & $\frac{\left(1-T_{j}\right)^{2}}{4}$ & $T_{j}\left(\frac{1-T_{j}}{2}\right)$ \\
\hline$\left(\mathrm{Y}_{\mathrm{ll}}\right)$ & $\leq \frac{1-T_{j}}{2}$ & $\leq \frac{1-T_{i}}{2}$ & $T_{i}$ & $T_{j}$ & $T_{i}\left(1-T_{i}-T_{j}\right)$ & $T_{j}\left(1-T_{i}-T_{j}\right)$
\end{tabular}

Table 2.2: Second-period equilibrium outcomes.

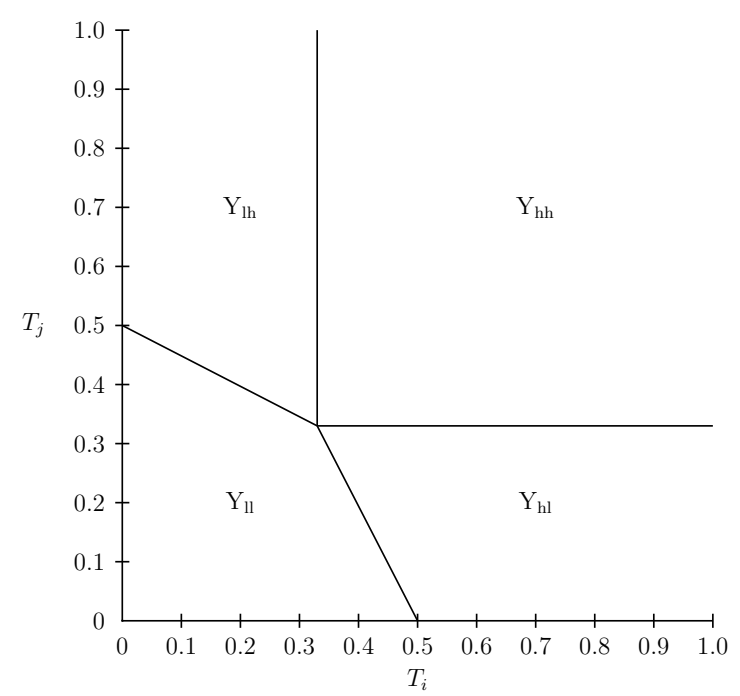

Figure 2.2: Second-period equilibrium regions for $\delta=0.5$. 
We now replace the second-period subgames by the second-period outcomes as induced by $f^{*}$. The result is a one-period reduced game with payoffs given by

$$
\Pi_{i}^{\mathrm{R}}\left(q_{i}, q_{j}\right)=\Pi_{i}\left(q_{i}, q_{j}, f_{i}^{*}\left(q_{i}, q_{j}\right), f_{j}^{*}\left(q_{i}, q_{j}\right)\right), \quad 0 \leq q_{i} \leq S_{i}, 0 \leq q_{j} \leq S_{j} .
$$

It follows that the reduced profit function of firm $i$ is given by

$$
\begin{aligned}
\Pi_{i}^{\mathrm{R}}\left(q_{i}, q_{j}\right)= & q_{i}\left(1-q_{i}-q_{j}\right)+ \\
& \left\{\begin{array}{lll}
\frac{1}{9} \delta, & \text { if } T_{i}>\frac{1}{3} \text { and } T_{j}>\frac{1}{3}, & \left(\mathrm{Y}_{\mathrm{hh}}\right) \\
\frac{1}{2} \delta T_{i}\left(1-T_{i}\right), & \text { if } 1-2 T_{j}<T_{i} \leq \frac{1}{3}, & \left(\mathrm{Y}_{\mathrm{lh}}\right) \\
\frac{1}{4} \delta\left(1-T_{j}\right)^{2}, & \text { if } T_{i}>\frac{1}{2}-\frac{1}{2} T_{j} \text { and } T_{j} \leq \frac{1}{3}, & \left(\mathrm{Y}_{\mathrm{hl}}\right) \\
\delta T_{i}\left(1-T_{i}-T_{j}\right), & \text { if } T_{i} \leq \min \left\{\frac{1}{2}-\frac{1}{2} T_{j}, 1-2 T_{j}\right\} . & \left(\mathrm{Y}_{\mathrm{ll}}\right)
\end{array}\right.
\end{aligned}
$$

A pair of strategies $\left(q_{1}^{*}, q_{2}^{*}\right)$ is a Nash equilibrium of the reduced game iff it holds that

$$
\begin{aligned}
& \Pi_{1}^{\mathrm{R}}\left(q_{1}^{*}, q_{2}^{*}\right) \geq \Pi_{1}^{\mathrm{R}}\left(q_{1}, q_{2}^{*}\right), \text { for all } q_{1} \in\left[0, S_{1}\right], \\
& \Pi_{2}^{\mathrm{R}}\left(q_{1}^{*}, q_{2}^{*}\right) \geq \Pi_{2}^{\mathrm{R}}\left(q_{1}^{*}, q_{2}\right), \text { for all } q_{2} \in\left[0, S_{2}\right] .
\end{aligned}
$$

A Nash equilibrium $\left(q_{1}^{*}, q_{2}^{*}\right)$ of the reduced game corresponds to a subgame perfect Nash equilibrium $\left(q_{1}^{*}, f_{1}^{*}, q_{2}^{*}, f_{2}^{*}\right)$ of the complete game and vice versa.

Lemma 2.4.1. $q_{i}^{*}, q_{j}^{*} \leq \frac{1}{2}$ for any Nash equilibrium $\left(q_{1}^{*}, q_{2}^{*}\right)$ of the reduced game.

Proof The first-period profit is $q_{i}\left(1-q_{i}-q_{j}\right)$, which is strictly decreasing in $q_{i}$ if $q_{i}>\frac{1}{2}-\frac{1}{2} q_{j}$, so in particular if $q_{i}>\frac{1}{2}$. If firm $i$ decreases its first-period sales, it increases its second-period stock. As can be seen in Table 2.2, firm $i$ 's second-period profit never decreases when its second-period stock increases. Consequently, firm $i$ strictly increases its profits if it sets $q_{i}=\frac{1}{2}$ instead of $q_{i}>\frac{1}{2}$.

Using the reduced profit function (2.2), we determine the reduced best responses, denoting by $\sigma_{i}^{\mathrm{R}}\left(q_{j}\right)$ the reduced best response of firm $i$ against $q_{j}$. Appendix A provides the computational details. Given $q_{j}$, the reduced profit function is not always concave, though it is continuous. As a consequence, the reduced best response against $q_{j}$ does always exist, but may not be unique. We therefore have a reduced best response correspondence 
rather than a reduced best response function. This correspondence may fail to be convex-valued though it is upper hemi-continuous. The reduced best response correspondence of firm $i$ is presented in Appendix A.

Quantities $\left(q_{1}^{*}, q_{2}^{*}\right)$ are a Nash equilibrium of the reduced game if and only if $q_{i}^{*} \in \sigma_{i}^{\mathrm{R}}\left(q_{j}^{*}\right)$ and $q_{j}^{*} \in \sigma_{j}^{\mathrm{R}}\left(q_{i}^{*}\right)$. The Nash equilibria of the reduced game, and thereby the subgame perfect Nash equilibria of the game of interest, are calculated in Appendix B. Since the reduced best response correspondences are not convex-valued, it is not guaranteed that a subgame perfect Nash equilibrium exists. Indeed, it turns out that for some combinations of $S_{i}, S_{j}$ and $\delta$ a subgame perfect Nash equilibrium fails to exist.

The set of exogenous variables $\left(S_{1}, S_{2}, \delta\right)$ can be partitioned in 12 regions. In each region in which there exists an equilibrium, the equilibria share the same qualitative features and are differentiable functions of $S_{1}, S_{2}$, and $\delta$. The equilibrium regions are given in Table 2.3 and depicted in Figure 2.3 for $\delta=0.5$. Table 2.3 also shows the equilibrium outcomes. We use the superscript ' $n c$ ' to refer to equilibria in the non-commitment case. The two letters in the subscript represent the relative level of stock of respectively firm $i$ and $j$, where 1 stands for low, $\mathrm{m}$ for medium, m' for medium-high and $\mathrm{h}$ for high.

As is illustrated by Figure 2.3 for $\delta=1 / 2$, the 12 regions are mutually exclusive. This property is generally true, leading to the following theorem.

Theorem 2.4.2. There is at most one subgame perfect Nash equilibrium for every combination of $S_{1}, S_{2}$, and $\delta$.

Proof It follows from comparing the constraints in Table 2.3, that all regions are disjoint. Therefore, every combination of $S_{i}, S_{j}$ and $\delta$ belongs to at most one equilibrium region. The Nash equilibrium of the reduced game is therefore unique for $\left(S_{i}, S_{j}, \delta\right)$ in Regions $\mathrm{X}_{\mathrm{ll}}^{\mathrm{nc}}$ up to and including $\mathrm{X}_{\mathrm{hh}}^{\mathrm{nc}}$. The reduced game has no Nash equilibrium for $\left(S_{i}, S_{j}, \delta\right)$ belonging to Region $\mathrm{X}_{\varnothing}^{\mathrm{nc}}$. Nash equilibria for the reduced game are in a one to one relationship with subgame perfect Nash equilibria of the complete game.

In some cases an equilibrium does not exist. ${ }^{5}$

Theorem 2.4.3. For every $\delta$, there is a set of stock levels $\left(S_{1}, S_{2}\right)$ with nonempty interior for which an equilibrium does not exist.

\footnotetext{
${ }^{5}$ More specifically, no pure equilibrium exists. Since the reduced strategy spaces are nonempty compact subsets of $\mathbb{R}$ and the payoff functions $\Pi_{i}^{\mathrm{R}}\left(q_{i}, q_{j}\right)$ are continuous (Glicksberg, 1952), there will be a mixed equilibrium.
} 
Chapter 2. Dynamic Duopoly with Intertemporal Capacity Constraints

\begin{tabular}{|c|c|c|c|}
\hline & Parameter conditions & Period 1 & Period 2 \\
\hline$\left(\mathrm{X}_{11}^{\mathrm{nc}}\right)$ & $\begin{array}{l}0 \leq S_{1}<\frac{1}{2}-\frac{1}{2} \delta-\frac{1}{2} S_{2} \\
0 \leq S_{2}<\frac{1}{2}-\frac{1}{2} \delta-\frac{1}{2} S_{1}\end{array}$ & $\begin{array}{l}q_{1}^{\mathrm{nc}}=S_{1} \\
q_{2}^{\mathrm{nc}}=S_{2}\end{array}$ & $\begin{array}{l}r_{1}^{\mathrm{nc}}=0 \\
r_{2}^{\mathrm{nc}}=0\end{array}$ \\
\hline$\left(\mathrm{X}_{\mathrm{lm}}^{\mathrm{nc}}\right)$ & $\begin{array}{l}0 \leq S_{1}<\frac{1}{3}(1-\delta) \\
\frac{1}{2}-\frac{1}{2} \delta-\frac{1}{2} S_{1} \leq S_{2} \leq 1-\frac{1}{2} S_{1}\end{array}$ & $\begin{array}{l}q_{1}^{\mathrm{nc}}=S_{1} \\
q_{2}^{\mathrm{nc}}=\frac{1-S_{1}+2 \delta S_{2}-\delta}{2+2 \delta}\end{array}$ & $\begin{array}{l}r_{1}^{\mathrm{nc}}=0 \\
r_{2}^{\mathrm{nc}}=\frac{2 S_{2}-1+S_{1}+\delta}{2+2 \delta}\end{array}$ \\
\hline$\left(\mathrm{X}_{\mathrm{ml}}^{\mathrm{nc}}\right)$ & $\begin{array}{l}\frac{1}{2}-\frac{1}{2} \delta-\frac{1}{2} S_{2} \leq S_{1} \leq 1-\frac{1}{2} S_{2} \\
0 \leq S_{2}<\frac{1}{3}(1-\delta)\end{array}$ & $\begin{array}{l}q_{1}^{\mathrm{nc}}=\frac{1-S_{2}+2 \delta S_{1}-\delta}{2+2 \delta} \\
q_{2}^{\mathrm{nc}}=S_{2}\end{array}$ & $\begin{array}{l}r_{2}^{\mathrm{nc}}=\frac{2 S_{1}-1+S_{2}+\delta}{2+2 \delta} \\
r_{2}^{\mathrm{nc}}=0\end{array}$ \\
\hline$\left(\mathrm{X}_{\mathrm{lh}}^{\mathrm{nc}}\right)$ & $\begin{array}{l}0 \leq S_{1}<\frac{1}{3}(1-\delta) \\
S_{2}>1-\frac{1}{2} S_{1}\end{array}$ & $\begin{array}{l}q_{1}^{\mathrm{nc}}=S_{1} \\
q_{2}^{\mathrm{nc}}=\frac{1}{2}-\frac{1}{2} S_{1}\end{array}$ & $\begin{array}{l}r_{1}^{\mathrm{nc}}=0 \\
r_{2}^{\mathrm{nc}}=\frac{1}{2}\end{array}$ \\
\hline$\left(\mathrm{X}_{\mathrm{hl}}^{\mathrm{nc}}\right)$ & $\begin{array}{l}S_{1}>1-\frac{1}{2} S_{2} \\
0 \leq S_{2}<\frac{1}{3}(1-\delta)\end{array}$ & $\begin{array}{l}q_{1}^{\mathrm{nc}}=\frac{1}{2}-\frac{1}{2} S_{2} \\
q_{2}^{\mathrm{nc}}=S_{2}\end{array}$ & $\begin{array}{l}r_{1}^{\mathrm{nc}}=\frac{1}{2} \\
r_{2}^{\mathrm{nc}}=0\end{array}$ \\
\hline$\left(\mathrm{X}_{\mathrm{mm}}^{\mathrm{nc}} a\right)$ & $\begin{array}{l}\frac{1}{3}(1-\delta) \leq S_{1} \leq \beta_{1} \\
\frac{1}{3}(1-\delta) \leq S_{2} \leq \beta_{2}\end{array}$ & \multirow{2}{*}{$\begin{array}{l}q_{1}^{\mathrm{nc}}=\frac{1-\delta+3 \delta S_{1}}{3+3 \delta} \\
q_{2}^{\mathrm{nc}}=\frac{1-\delta+3 \delta S_{2}}{3+3 \delta}\end{array}$} & \multirow{2}{*}{$\begin{array}{l}r_{1}^{\mathrm{nc}}=\frac{3 S_{1}-1+\delta}{3+3 \delta} \\
r_{2}^{\mathrm{nc}}=\frac{3 S_{2}-1+\delta}{3+3 \delta}\end{array}$} \\
\hline$\left(\mathrm{X}_{\mathrm{mm}}^{\mathrm{nc}} \mathrm{b}\right)$ & $\begin{array}{l}\beta_{3}<S_{1} \leq 1-\frac{1}{2} S_{2} \\
\beta_{4}<S_{2} \leq 1-\frac{1}{2} S_{1}\end{array}$ & & \\
\hline$\left(\mathrm{X}_{\mathrm{mh}}^{\mathrm{nc}}\right)$ & $\begin{array}{l}\frac{1}{3}(1-\delta) \leq S_{1} \leq \frac{2}{3}-\frac{1}{9} \delta \\
S_{2}>\beta_{5}\end{array}$ & $\begin{array}{l}q_{1}^{\mathrm{nc}}=\frac{1-\delta+2 \delta S_{1}}{3+2 \delta} \\
q_{2}^{\mathrm{nc}}=\frac{2+3 \delta-2 \delta S_{1}}{6+4 \delta}\end{array}$ & $\begin{array}{l}r_{1}^{\mathrm{nc}}=\frac{3 S_{1}-1+\delta}{3+2 \delta} \\
r_{2}^{\mathrm{nc}}=\frac{4+\delta-3 S_{1}}{6+4 \delta}\end{array}$ \\
\hline$\left(\mathrm{X}_{\mathrm{hm}}^{\mathrm{nc}}\right)$ & $\begin{array}{l}S_{1}>\beta_{6} \\
\frac{1}{3}(1-\delta) \leq S_{2} \leq \frac{2}{3}-\frac{1}{9} \delta\end{array}$ & $\begin{array}{l}q_{1}^{\mathrm{nc}}=\frac{2+3 \delta-2 \delta S_{2}}{6+4 \delta} \\
q_{2}^{\mathrm{nc}}=\frac{1-\delta+2 \delta S_{2}}{3+2 \delta}\end{array}$ & $\begin{array}{l}r_{1}^{\mathrm{nc}}=\frac{4+\delta-3 S_{2}}{6+4 \delta} \\
r_{2}^{\mathrm{nc}}=\frac{3 S_{2}-1+\delta}{3+2 \delta}\end{array}$ \\
\hline$\left(\mathrm{X}_{\mathrm{m}^{\prime} \mathrm{h}}^{\mathrm{nc}}\right)$ & $\begin{array}{l}\frac{2}{3}-\frac{1}{9} \delta<S_{1} \leq \frac{2}{3} \\
S_{2}>\beta_{7}\end{array}$ & $\begin{array}{l}q_{1}^{\mathrm{nc}}=S_{1}-\frac{1}{3} \\
q_{2}^{\mathrm{nc}}=\frac{2}{3}-\frac{1}{2} S_{1}\end{array}$ & $\begin{array}{l}r_{1}^{\mathrm{nc}}=\frac{1}{3} \\
r_{2}^{\mathrm{nc}}=\frac{1}{3}\end{array}$ \\
\hline$\left(\mathrm{X}_{\mathrm{hm}^{\prime}}^{\mathrm{nc}}\right)$ & $\begin{array}{l}S_{1}>\beta_{8} \\
\frac{2}{3}-\frac{1}{9} \delta<S_{2} \leq \frac{2}{3}\end{array}$ & $\begin{array}{l}q_{1}^{\mathrm{nc}}=\frac{2}{3}-\frac{1}{2} S_{2} \\
q_{2}^{\mathrm{nc}}=S_{2}-\frac{1}{3}\end{array}$ & $\begin{array}{l}r_{1}^{\mathrm{nc}}=\frac{1}{3} \\
r_{2}^{\mathrm{nc}}=\frac{1}{3}\end{array}$ \\
\hline$\left(\mathrm{X}_{\mathrm{hh}}^{\mathrm{nc}}\right)$ & $\begin{array}{l}S_{1}>\frac{2}{3} \\
S_{2}>\frac{2}{3}\end{array}$ & $\begin{array}{l}q_{1}^{\mathrm{nc}}=\frac{1}{3} \\
q_{2}^{\mathrm{nc}}=\frac{1}{3}\end{array}$ & $\begin{array}{l}r_{1}^{\mathrm{nc}}=\frac{1}{3} \\
r_{2}^{\mathrm{nc}}=\frac{1}{3}\end{array}$ \\
\hline$\left(\mathrm{X}_{\varnothing}^{\mathrm{nc}}\right)$ & All other values of $\left(S_{1}, S_{2}, \delta\right)$ & No equilibrium & \\
\hline
\end{tabular}

Explanation of the symbols

\begin{tabular}{l|l}
$\beta_{1}$ & $\frac{7}{6}-S_{2}-\frac{1}{6} \delta+\frac{\sqrt{(1+\delta)\left(1+\frac{1}{2} \delta\right)}}{1+\delta}\left(\frac{5}{6}+\frac{1}{6} \delta-S_{2}\right)$ \\
$\beta_{2}$ & $\frac{7}{6}-S_{1}-\frac{1}{6} \delta+\frac{\sqrt{(1+\delta)\left(1+\frac{1}{2} \delta\right)}}{1+\delta}\left(\frac{5}{6}+\frac{1}{6} \delta-S_{1}\right)$ \\
$\beta_{3}$ & $\frac{4+5 \frac{1}{2} \delta-\frac{1}{2} \delta^{2}-3 \delta S_{2}}{6+6 \delta}$ \\
$\beta_{4}$ & $\frac{4+5 \frac{1}{2} \delta-\frac{1}{2} \delta^{2}-3 \delta S_{1}}{6+6 \delta}$ \\
$\beta_{5}$ & $\frac{7+6 \frac{1}{2} \delta+\frac{3}{2} \delta^{2}-6 S_{1}-5 \delta S_{1}-\delta^{2} S_{1}+\left(5+5 \delta-2 \delta S_{1}\right) \sqrt{(1+\delta)\left(1+\frac{1}{2} \delta\right)}}{6+7 \delta+2 \delta^{2}+(6+4 \delta) \sqrt{(1+\delta)\left(1+\frac{1}{2} \delta\right)}}$ \\
$\beta_{6}$ & $\frac{7+6 \frac{1}{2} \delta+\frac{3}{2} \delta^{2}-6 S_{2}-5 \delta S_{2}-\delta^{2} S_{2}+\left(5+5 \delta-2 \delta S_{2}\right) \sqrt{(1+\delta)\left(1+\frac{1}{2} \delta\right)}}{6+7 \delta+2 \delta^{2}+(6+4 \delta) \sqrt{(1+\delta)\left(1+\frac{1}{2} \delta\right)}}$ \\
$\beta_{7}$ & $\frac{10+6 \delta-7 S_{1}-3 \delta S_{1}}{8+6 \delta}$ \\
$\beta_{8}$ & $\frac{10+6 \delta-7 S_{2}-3 \delta S_{2}}{8+6 \delta}$
\end{tabular}

Table 2.3: Equilibria in the non-commitment case. 


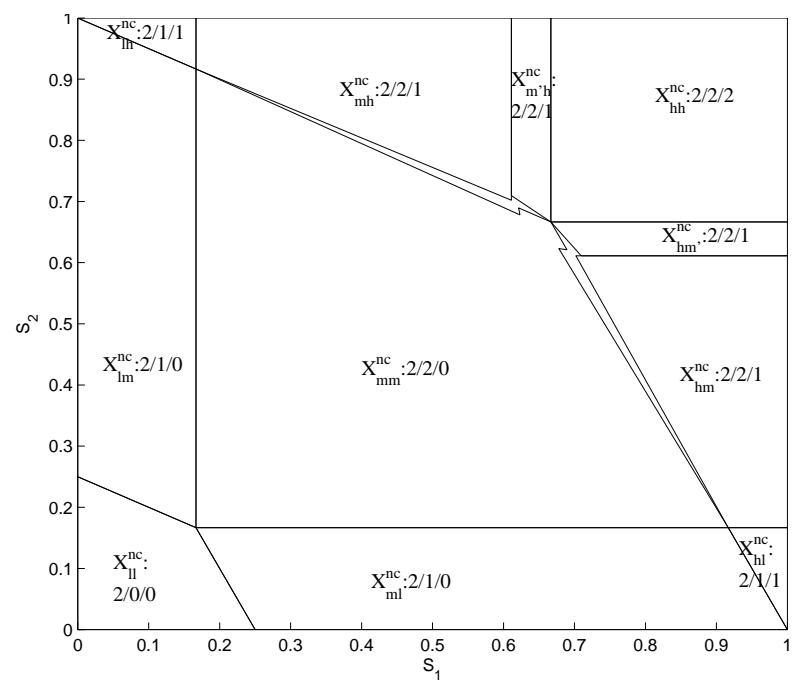

Figure 2.3: The non-commitment case: Equilibrium outcome regions and the number of firms that have stock in respectively period 1 , period 2 and after period 2 , for $\delta=0.5$.

Proof It can be verified that for each $\delta$, the set of stock profiles $\left(S_{1}, S_{2}\right)$ such that $\left(S_{1}, S_{2}, \delta\right)$ belongs to Region $\mathrm{X}_{\varnothing}^{\text {nc }}$ has a non-empty interior.

Figure 2.3 gives an overview of the dynamic development of the market structure, where again $N_{1} / N_{2} / N_{r}$ represents the number of firms that sell the commodity in the first period, the number of firms that sell the commodity in the second period, and the number of firms that have residual stock by the end of the second period. Just as in the non-commitment case, the number of active firms increases when initial production levels increase.

Some of the regions in the non-commitment case coincide with those in the case with commitment. This holds specifically for the Regions $\mathrm{X}_{11}^{\mathrm{nc}}, \mathrm{X}_{\mathrm{lm}}^{\mathrm{nc}}$, $\mathrm{X}_{\mathrm{ml}}^{\mathrm{nc}}, \mathrm{X}_{\mathrm{lh}}^{\mathrm{nc}}, \mathrm{X}_{\mathrm{hl}}^{\mathrm{nc}}, \mathrm{X}_{\mathrm{mm}}^{\mathrm{nc}}$, and $\mathrm{X}_{\mathrm{hh}}^{\mathrm{nc}}{ }^{6}$ For these regions, the equilibrium outcomes in the commitment and in the non-commitment case are equivalent.

In the commitment case, the price never decreases from period 1 to period 2. The reason is that a decreasing price would make it profitable for a firm to transfer some of its sales from period 2 to period 1 . This line of

\footnotetext{
${ }^{6}$ We have named the regions in the non-commitment case in such a way that the names in the commitment and non-commitment case coincide as much as possible.
} 
reasoning does not hold when there is no commitment. Indeed, in the noncommitment case a transfer of sales from period 2 to period 1 may trigger a reaction by the competing firm, which renders such a transfer unprofitable, even when the price in period 1 is higher than in period 2 .

Proposition 2.4.4. In Regions $\mathrm{X}_{l l}^{\mathrm{nc}}, \mathrm{X}_{l m}^{\mathrm{nc}}, \mathrm{X}_{m l}^{\mathrm{nc}}, \mathrm{X}_{l h}^{\mathrm{nc}}, \mathrm{X}_{h l}^{\mathrm{nc}}, \mathrm{X}_{m m}^{\mathrm{nc}}$ and $\mathrm{X}_{h h}^{\mathrm{nc}}$, the equilibrium price weakly increases over time. For any $\delta$, there is a set of stock levels $\left(S_{1}, S_{2}\right) \in \mathrm{X}_{m h}^{\mathrm{nc}} \cup \mathrm{X}_{m \prime h}^{\mathrm{nc}} \cup \mathrm{X}_{h m}^{\mathrm{nc}} \cup \mathrm{X}_{h m}^{\mathrm{nc}}$, with non-empty interior such that the equilibrium price strictly decreases over time. In particular, the equilibrium price strictly decreases over time if and only if $S_{i}<S_{j}$ and

$$
\begin{gathered}
\frac{2-2 \delta}{3-2 \delta} \leq S_{i}<\frac{2}{3}-\frac{1}{9} \delta \\
S_{j}>\frac{7+6 \frac{1}{2} \delta+\frac{3}{2} \delta^{2}-6 S_{i}-5 \delta S_{i}-\delta^{2} S_{i}+\left(5+5 \delta-2 \delta S_{i}\right) \sqrt{(1+\delta)\left(1+\frac{1}{2} \delta\right)}}{6+7 \delta+2 \delta^{2}+(6+4 \delta) \sqrt{(1+\delta)\left(1+\frac{1}{2} \delta\right)}}
\end{gathered}
$$

or

$$
\begin{gathered}
\frac{2}{3}-\frac{1}{9} \delta<S_{i}<\frac{2}{3}, \\
S_{j}>\frac{10+6 \delta-7 S_{i}-3 \delta S_{i}}{8+6 \delta} .
\end{gathered}
$$

Proof By Proposition 2.3.1, price never decreases over time in the commitment situation. Price decreases in the non-commitment case are therefore only possible in regions where the non-commitment case is different from the case with commitment, i.e. Regions $\mathrm{X}_{\mathrm{mh}}^{\mathrm{nc}}, \mathrm{X}_{\mathrm{hm}}^{\mathrm{nc}}, \mathrm{X}_{\mathrm{m} \text { 'h }}^{\mathrm{nc}}$, and $\mathrm{X}_{\mathrm{hm}}^{\mathrm{nc}}$. In these regions, one firm has an intermediate and one firm has a high stock level. Let $i$ be the intermediate firm and let $j$ be the large firm. In Regions $\mathrm{X}_{\mathrm{mh}}^{\mathrm{nc}}$ and $\mathrm{X}_{\mathrm{hm}}^{\mathrm{nc}}$ it holds that

$$
\begin{aligned}
\frac{1}{3}(1-\delta) & <S_{i} \leq \frac{2}{3}-\frac{1}{9} \delta \\
S_{j} & >\frac{7+6 \frac{1}{2} \delta+\frac{3}{2} \delta^{2}-6 S_{i}-5 \delta S_{i}-\delta^{2} S_{i}+\left(5+5 \delta-2 \delta S_{i}\right) \sqrt{(1+\delta)\left(1+\frac{1}{2} \delta\right)}}{6+7 \delta+2 \delta^{2}+(6+4 \delta) \sqrt{(1+\delta)\left(1+\frac{1}{2} \delta\right)}} .
\end{aligned}
$$

The total quantity sold in the first period is

$$
q_{i}^{\mathrm{nc}}+q_{j}^{\mathrm{nc}}=\frac{1-\delta+2 \delta S_{i}}{3+2 \delta}+\frac{2+3 \delta-2 \delta S_{i}}{6+4 \delta}=\frac{4+\delta+2 \delta S_{i}}{6+4 \delta} .
$$


The total quantity sold in the second period is

$$
r_{i}^{\mathrm{nc}}+r_{j}^{\mathrm{nc}}=\frac{3 S_{i}-1+\delta}{3+2 \delta}+\frac{4+\delta-3 S_{i}}{6+4 \delta}=\frac{3 S_{i}+2+3 \delta}{6+4 \delta} .
$$

The price strictly decreases from period 1 to period 2 when $4+\delta+2 \delta S_{i}<$ $3 S_{i}+2+3 \delta$, so when $S_{i}>\frac{2-2 \delta}{3-2 \delta}$.

In Regions $\mathrm{X}_{\mathrm{m}{ }^{\prime} \mathrm{h}}^{\mathrm{nc}}$ and $\mathrm{X}_{\mathrm{hm}}^{\mathrm{nc}}$, we have

$$
\begin{aligned}
\frac{2}{3}-\frac{1}{9} \delta & <S_{i} \leq \frac{2}{3} \\
S_{j} & >\frac{10+6 \delta-7 S_{i}-3 \delta S_{i}}{8+6 \delta} .
\end{aligned}
$$

The total quantity sold in the first period is

$$
q_{i}^{\mathrm{nc}}+q_{j}^{\mathrm{nc}}=S_{i}-\frac{1}{3}+\frac{2}{3}-\frac{1}{2} S_{i}=\frac{1}{2} S_{i}+\frac{1}{3} .
$$

The total quantity sold in the second period is

$$
r_{i}^{\mathrm{nc}}+r_{j}^{\mathrm{nc}}=\frac{1}{3}+\frac{1}{3}=\frac{2}{3}
$$

Since $\frac{1}{2} S_{i}+\frac{1}{3}<\frac{2}{3}$ as long as $S_{i}<\frac{2}{3}$, in this region, price strictly decreases from period one to period two whenever $S_{i} \neq \frac{2}{3}$.

Proposition 2.4.4 makes clear that price may decrease over time in the non-commit-ment case. This can happen for the following reason. In the settings where price decreases over time, the larger firm reacts in both periods - unrestricted by its stock - per-period optimal to the sales of the smaller firm. Since the smaller firm has a stock less than $1 / 3$ in the second period, the larger firm cannot deviate in the first period in such a way that the smaller firm will lower its second-period sales. This implies that the larger firm cannot increase profits by deviating. The smaller firm, just as in the commitment case, might want to transfer some of its sales from the second to the first period. However, in the non-commitment situation, if the smaller firm transfers sales from period 2 to period 1, there will be a response by the larger firm. The larger firm reacts to this transfer by increasing its second-period sales, causing the second-period price to fall. Therefore, the second-period profits of the smaller firm drop. The decrease in profits in the second period outweigh the increase in profits in the first period. This makes 
transferring sales from the second-period to the first not worth the while for the smaller firm.

In the commitment case, an increase in a firm's stock leads to an increase in profits. Is this property still true in the non-commitment case? It is easily shown, with the help of the derivatives of the equilibrium profits, that within each region profit rises when a firm's stock level increases. Moreover, the profit function is continuous on the domain of $\left(S_{i}, S_{j}, \delta\right)$ for which an equilibrium exists. However, it is still possible for the profit to decrease when a firm's stock level increases, namely when a small increase in stock level leads to non-existence of equilibrium. The next proposition confirms that such decreases in profit may occur for specific parameter values. That is, equilibria may not be "destroy-proof".

Proposition 2.4.5. An increase in $S_{i}$, ceteris paribus, leads to a weak increase of the equilibrium profit of firm $i$, as long as the increase doesn't change the equilibrium outcome region. If an increase in $S_{i}$ does change the equilibrium outcome region, there are combinations of $S_{i}, S_{j}$ and $\delta$ such that an increase in $S_{i}$ leads to a strict decrease in equilibrium profit of firm $i$.

Proof The derivative of the equilibrium profit function with respect to $S_{i}$ is non-negative in every equilibrium region. The non-existence of an equilibrium for some combinations of $\left(S_{i}, S_{j}, \delta\right)$ makes it possible that a strict increase in $S_{i}$ leads to a strict decrease in the equilibrium profit of firm $i$. Take $\delta=0.2, S_{j}=0.69824$, and

$$
S_{i}=\frac{7}{6}-S_{j}-\frac{1}{6} \delta+\frac{\sqrt{(1+\delta)\left(1+\frac{1}{2} \delta\right)}}{1+\delta}\left(\frac{5}{6}+\frac{1}{6} \delta-S_{j}\right) \approx 0.59634 .
$$

These parameters correspond to a point on the upper boundary of Region $\mathrm{X}_{\mathrm{mm}}^{\mathrm{nc}}$. The equilibrium profit for firm $i$ equals 0.12937 . We now let $S_{i}$ increase to

$$
\begin{aligned}
S_{i}^{\prime} & =\frac{7+6 \frac{1}{2} \delta+\frac{3}{2} \delta^{2}-6 S_{j}-78 \delta S_{j}-2 \delta^{2} S_{j}+\left(5+5 \delta-6 S_{j}-4 \delta S_{j}\right) \sqrt{(1+\delta)\left(1+\frac{1}{2} \delta\right)}}{6+5 \delta+\delta^{2}+2 \delta \sqrt{(1+\delta)\left(1+\frac{1}{2} \delta\right)}} \\
& \approx 0.61011 .
\end{aligned}
$$

Our parameters now belong to Region $\mathrm{X}_{\mathrm{mh}}^{\mathrm{nc}}$. The equilibrium profit for firm $i$ equals 0.12751 .

We now study the consequences of increasing difference in stock size on sales. 
Proposition 2.4.6. Given fixed aggregate stock $S_{1}+S_{2}$, an increase in $\mid S_{1}-$ $S_{2}$ l leads to a weak decrease in first-period aggregate equilibrium sales and a weak increase of first-period equilibrium price. It leads to a decrease in second-period aggregate equilibrium sales and an increase in second-period equilibrium price in Regions $\mathrm{X}_{m h}^{\mathrm{c}}$ and $\mathrm{X}_{h m}^{\mathrm{c}}$ and to an increase in secondperiod aggregate equilibrium sales and a decrease in second-period equilibrium price in Regions $\mathrm{X}_{l m}^{\mathrm{c}}$ and $\mathrm{X}_{m l}^{\mathrm{c}}$. It has no effect on second-period aggregate equilibrium sales and equilibrium price in the other regions.

Proof Let $S=S_{1}+S_{2}$ be fixed and assume without loss of generality that $S_{2} \geq S_{1}$. Then $\left|S_{1}-S_{2}\right|$ increases if $S_{1}$ decreases. Since $S_{2} \geq S_{1}$, it holds that $\left(S_{1}, S_{2}, \delta\right) \in \mathrm{X}_{\mathrm{ll}}^{\mathrm{nc}} \cup \mathrm{X}_{\mathrm{lm}}^{\mathrm{nc}} \cup \mathrm{X}_{\mathrm{lh}}^{\mathrm{nc}} \cup \mathrm{X}_{\mathrm{mm}}^{\mathrm{nc}} \cup \mathrm{X}_{\mathrm{mh}}^{\mathrm{nc}} \cup \mathrm{X}_{\mathrm{m}^{\prime} \mathrm{h}}^{\mathrm{nc}} \cup \mathrm{X}_{\mathrm{hh}}^{\mathrm{nc}}$. It holds that

$$
\begin{array}{ll}
Q_{\mathrm{ll}}^{\mathrm{nc}}=S, & \frac{\partial Q_{11}^{\mathrm{nc}}}{\partial S_{1}}=0, \\
Q_{\mathrm{lm}}^{\mathrm{nc}}=\frac{1-\delta+S_{1}+2 \delta S}{2+2 \delta}, & \frac{\partial Q_{\mathrm{lm}}^{\mathrm{cc}}}{\partial S_{1}}=\frac{1}{2+2 \delta}>0, \\
Q_{\mathrm{lh}}^{\mathrm{nc}}=\frac{1}{2}+\frac{1}{2} S_{1}, & \frac{\partial Q_{\mathrm{lh}}^{\mathrm{nc}}}{\partial S_{1 \mathrm{c}}}=\frac{1}{2}>0, \\
Q_{\mathrm{mm}}^{\mathrm{nc}}=\frac{2-2 \delta+3 \delta S}{3+3 \delta}, & \frac{\partial Q_{\mathrm{mm}}^{1 \mathrm{c}}}{\partial S_{1}}=0, \\
Q_{\mathrm{mh}}^{\mathrm{nc}}=\frac{4+\delta+2 \delta S_{1}}{6+4 \delta}, & \frac{\partial Q_{\mathrm{mh}}^{\mathrm{n}}}{\partial S_{1}}=\frac{2 \delta}{6+4 \delta}>0, \\
Q_{\mathrm{m} h}^{\mathrm{nc}}=\frac{1}{2} S_{1}+\frac{1}{3}, & \frac{\partial Q_{\mathrm{m}}^{\mathrm{nch}}}{\partial S_{1}}=\frac{1}{2}>0 . \\
Q_{\mathrm{hh}}^{\mathrm{nc}}=\frac{2}{3}, & \frac{\partial Q_{\mathrm{hh}}^{\mathrm{nc}}}{\partial S_{1}}=0,
\end{array}
$$

Between Regions $\mathrm{X}_{\mathrm{mm}}^{\mathrm{nc}}$ and $\mathrm{X}_{\mathrm{mh}}^{\mathrm{nc}}$ there is no equilibrium. Consider an increase in $S_{1}$ together with a decrease of the same magnitude in $S_{2}$ that leads to a move from Region $\mathrm{X}_{\mathrm{mh}}^{\mathrm{nc}}$ to Region $\mathrm{X}_{\mathrm{mm}}^{\mathrm{nc}}$. It holds that $Q_{\mathrm{mm}}^{\mathrm{nc}}$ coincides with $Q_{\mathrm{mm}}^{\mathrm{c}}$ and

$$
Q_{\mathrm{mh}}^{\mathrm{nc}}=\frac{4+\delta+2 \delta S_{1}}{6+4 \delta}<\frac{4+2 \delta+3 \delta S_{1}}{6+6 \delta}=Q_{\mathrm{mh}}^{\mathrm{c}} .
$$

The desired result for this case now follows from Proposition 2.3.3.

Between Regions $\mathrm{X}_{\mathrm{m}^{\prime} \mathrm{h}}^{\mathrm{nc}}$ and $\mathrm{X}_{\mathrm{mm}}^{\mathrm{nc}}$ there is no equilibrium. Consider an increase in $S_{1}$ together with a decrease of the same magnitude in $S_{2}$ that leads to a move from Region $\mathrm{X}_{\mathrm{m}^{\prime} \mathrm{h}}^{\mathrm{nc}}$ to Region $\mathrm{X}_{\mathrm{mm}}^{\mathrm{nc}}$. Again, it holds that $Q_{\mathrm{mm}}^{\mathrm{nc}}$ coincides with $Q_{\mathrm{mm}}^{\mathrm{c}}$, Region $\mathrm{X}_{\mathrm{m}^{\prime} \mathrm{h}}^{\mathrm{nc}}$ is a subset of Region $\mathrm{X}_{\mathrm{mh}}^{\mathrm{c}}$, and

$$
Q_{\mathrm{m} \mathrm{h}^{\prime} \mathrm{h}}^{\mathrm{nc}}=\frac{1}{2} S_{1}+\frac{1}{3} \leq \frac{4+2 \delta+3 \delta S_{1}}{6+6 \delta}=Q_{\mathrm{mh}}^{\mathrm{c}}
$$

where $S_{1} \leq 2 / 3$ is used to derive the inequality sign. The desired result for this case now follows from Proposition 2.3.3. 
For second-period aggregate sales we have that

$$
\begin{aligned}
& Q_{\mathrm{ll}}^{\mathrm{nc}}=0, \quad \frac{\partial Q_{11}^{\mathrm{nc}}}{\partial S_{1 \mathrm{l}}}=0, \\
& Q_{\mathrm{lm}}^{\mathrm{nc}}=\frac{2 S-S_{1}-1+\delta}{2+2 \delta}, \quad \frac{\partial Q_{1 \mathrm{~m}}^{\mathrm{nc}}}{\partial S_{1 \mathrm{c}}}=-\frac{1}{2+2 \delta}<0, \\
& Q_{\mathrm{lh}}^{\mathrm{nc}}=\frac{1}{2}, \quad \frac{\partial Q_{\mathrm{lh}}^{\mathrm{nc}}}{\partial S_{1 \mathrm{c}}}=0, \\
& Q_{\mathrm{mm}}^{\mathrm{nc}}=\frac{3 S-2+2 \delta}{3+3 \delta}, \quad \frac{\partial Q_{\mathrm{mm}}^{1 \mathrm{~m}}}{\partial S_{1}}=0, \\
& Q_{\mathrm{mh}}^{\mathrm{nc}}=\frac{3 S_{1}+2+3 \delta}{6+4 \delta}, \quad \frac{\partial Q_{\mathrm{mh}}^{\mathrm{nc}}}{\partial S_{1}}=\frac{3}{6+4 \delta}>0, \\
& Q_{\mathrm{m} \mathrm{m}^{\prime} \mathrm{h}}^{\mathrm{nc}}=\frac{2}{3}, \quad \frac{\partial Q_{\mathrm{m}^{\prime} \mathrm{h}}^{\mathrm{nc}}}{\partial S_{1}}=0, \\
& Q_{\mathrm{hh}}^{\mathrm{nc}}=\frac{2}{3}, \quad \frac{\partial Q_{\mathrm{hh}}^{\mathrm{nc}}}{\partial S_{1}}=0 .
\end{aligned}
$$

Between Regions $\mathrm{X}_{\mathrm{mm}}^{\mathrm{nc}}$ and $\mathrm{X}_{\mathrm{mh}}^{\mathrm{nc}}$ there is no equilibrium. For this region, the consequences of increasing disparity of initial stock on second-period aggregate sales haven't been discussed yet.

Proposition 2.4.7. Consider an increase in $S_{1}$ together with a decrease of the same magnitude in $S_{2}$ that leads to a move from Region $X_{m h}^{\mathrm{nc}}$ to Region $X_{m m}^{\mathrm{nc}}$. The second-period equilibrium sales may both decrease and increase, depending on the values of $S_{1}, S_{2}$, and $\delta$.

Proof For instance, when $S_{1}=5 / 9, S_{2}=3 / 4$, and $\delta=1$, we are in Region $\mathrm{X}_{\mathrm{mh}}^{\mathrm{nc}}$ and the aggregate second-period sales are equal to 2/3. After an increase in $S_{1}$ accompanied by a decrease in $S_{2}$ of the same magnitude resulting in $S_{1}=S_{2}=47 / 72$, we are in Region $\mathrm{X}_{\mathrm{mm}}^{\mathrm{nc}}$ and the aggregate second-period sales are equal to $47 / 72<2 / 3$. We now make the same calculations for a discount rate equal to $1 / 2$. When $\left(S_{1}, S_{2}, \delta\right)=(5 / 9,3 / 4,1 / 2)$ we are in Region $\mathrm{X}_{\mathrm{mh}}^{\mathrm{nc}}$ and the aggregate second-period sales are equal to $13 / 24$, whereas at $\left(S_{1}, S_{2}, \delta\right)=$ $(47 / 72,47 / 72,1 / 2)$ we are in Region $X_{\mathrm{mm}}^{\mathrm{nc}}$ and the aggregate second-period sales are equal to $35 / 54>13 / 24$.

So, in these settings, increasing disparity of initial stock can increase as well as decrease second-period aggregate sales. There is also no equilibrium between Regions $\mathrm{X}_{\mathrm{mm}}^{\mathrm{nc}}$ and $\mathrm{X}_{\mathrm{m}{ }^{\prime} \mathrm{h}}^{\mathrm{nc}}$. Here, it can be shown that an increase in disparity of initial stock will always decrease second period sales.

Proposition 2.4.8. An increase in $S_{1}$ together with a decrease of the same size in $S_{2}$ that leads from Region $X_{m}^{\mathrm{nc} h}$ to Region $X_{m m}^{\mathrm{nc}}$ will univocally lead to a decrease in second-period sales. 
Proof In Region $\mathrm{X}_{\mathrm{mm}}^{\mathrm{nc}}$ it holds that $S_{1}+S_{2} \leq 4 / 3$, we have that

$$
Q_{\mathrm{mm}}^{\mathrm{nc}}=\frac{3 S-2+2 \delta}{3+3 \delta} \leq \frac{2}{3}=Q_{\mathrm{m}^{\prime} \mathrm{h}}^{\mathrm{nc}} .
$$

Analogous results hold for comparative statics involving Regions $\mathrm{X}_{\mathrm{mm}}^{\mathrm{nc}}$ and $\mathrm{X}_{\mathrm{hm}}^{\mathrm{nc}}$, and Regions $\mathrm{X}_{\mathrm{mm}}^{\mathrm{nc}}$ and $\mathrm{X}_{\mathrm{hm}}^{\mathrm{nc}}$.

We next evaluate the effect of an increase in stock on consumer surplus. We use the same measure for consumer surplus as before.

Proposition 2.4.9. An increase in $S_{i}$, ceteris paribus, leads to a weak increase in equilibrium consumer surplus, as long as the increase doesn't change the equilibrium outcome region. For some combinations of $S_{i}, S_{j}$ and $\delta$, a strict increase in $S_{i}$ does change the equilibrium outcome region. This can lead to a strict decrease in equilibrium consumer surplus.

Proof It follows directly from the equilibrium outcomes that per-period sales in every equilibrium outcome region weakly increase in $S_{1}$ and $S_{2}$. However, take $\delta, S_{i}, S_{i}^{\prime}$ and $S_{j}$ as defined in the proof of Proposition 2.4.5. Equilibrium consumer surplus for $\delta, S_{i}, S_{j}$ is

$$
C S_{\mathrm{mm}}^{\mathrm{nc}} \approx 0.25818 \text {. }
$$

An increase from $S_{i}$ to $S_{i}^{\prime}$ results in equilibrium consumer surplus of

$$
C S_{\mathrm{mh}}^{\mathrm{nc}} \approx 0.25600 \text {. }
$$

That is, just like the firms, consumers usually gain from an increase in stock. There are settings in which consumers are better off if a firm does not increase its stock. However, this can only happen if, for some stock levels in between the old and new stock level of the firm, ceteris paribus, an equilibrium doesn't exist.

The influence of increasing difference in stock level on consumer surplus is given in the following proposition.

Proposition 2.4.10. Given fixed aggregate stock $S_{1}+S_{2}$, an increase in $\mid S_{1}-$ $S_{2}$ l leads to a weak decrease in equilibrium consumer surplus. 
Proof By Proposition 2.3.5, this proposition holds for $\left|S_{1}-S_{2}\right|$, as long as $\left(S_{1}, S_{2}, \delta\right) \notin \mathrm{X}_{\mathrm{mh}}^{\mathrm{nc}} \cup \mathrm{X}_{\mathrm{hm}}^{\mathrm{nc}} \cup \mathrm{X}_{\mathrm{m}^{\prime} \mathrm{h}}^{\mathrm{nc}} \cup \mathrm{X}_{\mathrm{hm}}^{\mathrm{nc}}$. Assume, without loss of generality, that $S_{2} \geq S_{1}$, so $\left|S_{1}-S_{2}\right|$ increases if $S_{1}$ decreases. Proposition 2.4.6 implies a weak decrease in sales in both periods when $\left|S_{1}-S_{2}\right|$ increases, and therefore a weak decrease in consumer surplus, for Region $X_{m^{\prime} h}^{n c}$ and $X_{m h}^{n c}$. The remaining cases to check are those where a decrease in $S_{1}$ changes the equilibrium outcome from a point in $\mathrm{X}_{\mathrm{mm}}^{\mathrm{nc}}$ to a point in $\mathrm{X}_{\mathrm{mh}}^{\mathrm{nc}}$ or from $\mathrm{X}_{\mathrm{mm}}^{\mathrm{nc}}$ to $\mathrm{X}_{\mathrm{m}^{\prime} \mathrm{h}}^{\mathrm{nc}}$.

Let $c=S_{1}+S_{2}$, where $c$ is a constant. Consumer surplus in Region $\mathrm{X}_{\mathrm{mh}}^{\mathrm{nc}}$ and Region $\mathrm{X}_{\mathrm{m}^{\prime} \mathrm{h}}^{\mathrm{nc}}$ is

$$
\begin{aligned}
C S_{\mathrm{mh}}^{\mathrm{nc}} & =\frac{1}{2}\left(\frac{4+\delta+2 \delta S_{1}}{6+4 \delta}\right)^{2}+\frac{1}{2} \delta\left(\frac{3 S_{1}+2+3 \delta}{6+4 \delta}\right)^{2}, \\
C S_{\mathrm{m} \mathrm{m}^{\prime} \mathrm{h}}^{\mathrm{nc}} & =\frac{1}{2}\left(\frac{1}{2} S_{1}+\frac{1}{3}\right)^{2}+\frac{2}{9} \delta
\end{aligned}
$$

and in Region $\mathrm{X}_{\mathrm{mm}}^{\mathrm{nc}}$

$$
C S_{\mathrm{mm}}^{\mathrm{nc}}=\frac{1}{2}\left(\frac{2-2 \delta+3 \delta c}{3+3 \delta}\right)^{2}+\frac{1}{2} \delta\left(\frac{3 c-2+2 \delta}{3+3 \delta}\right)^{2} .
$$

As mentioned, consumer surplus in Regions $\mathrm{X}_{\mathrm{mh}}^{\mathrm{nc}}$ and $\mathrm{X}_{\mathrm{m}^{\prime} \mathrm{h}}^{\mathrm{nc}}$ increases with $S_{1}$, for fixed $c$. In Region $\mathrm{X}_{\mathrm{mm}}^{\mathrm{nc}}$, consumer surplus doesn't change if $S_{1}$ changes, for fixed $c$. This implies that, if $C S_{\mathrm{mh}}^{\mathrm{nc}} \leq C S_{\mathrm{mm}}^{\mathrm{nc}}$ for any $\left(S_{1}, S_{2}, \delta\right) \in$ $\left\{\left(S_{1}, S_{2}, \delta\right) \mid S_{2}=\beta_{5}, \frac{1}{3}(1-\delta) \leq S_{1} \leq \frac{2}{3}-\frac{1}{9} \delta\right\}$, consumer surplus decreases when an increase in $S_{1}$ changes the equilibrium outcome from period $\mathrm{X}_{\mathrm{mh}}^{\mathrm{nc}}$ to $\mathrm{X}_{\mathrm{mm}}^{\mathrm{nc}}$. Calculations indeed show that $C S_{\mathrm{mh}}^{\mathrm{nc}} \leq C S_{\mathrm{mm}}^{\mathrm{nc}}$ for these values of $\left(S_{1}, S_{2}, \delta\right)$. It also implies that, if $C S_{\mathrm{m} \text { h }}^{\mathrm{nc}} \leq C S_{\mathrm{mm}}^{\mathrm{nc}}$ for any $\left(S_{1}, S_{2}, \delta\right) \in\left\{\left(S_{1}, S_{2}, \delta\right) \mid S_{2}=\right.$ $\left.\beta_{7}, \frac{2}{3}-\frac{1}{9} \delta<S_{1} \leq \frac{2}{3}\right\}$, consumer surplus decreases when an increase in $S_{1}$ changes the equilibrium outcome from period $X_{m^{\prime} h}^{n c}$ to $X_{m m}^{n c}$.

The last part of this section is, again, devoted to total surplus. We have already seen that an increase in a firm's stock can lead to a decrease in its equilibrium profit and in consumer surplus. It will not come as a surprise that, with some extra calculations, the same type of results can be found for total surplus. If an increase in stock of one of the firms doesn't change the equilibrium outcome region, equilibrium total surplus increases with this increase in stock. If an increase in stock of one of the firms does change the equilibrium outcome region, for some combinations of variables, this leads to a decrease in total surplus. And, given fixed aggregate stock $S_{1}+S_{2}$, an increase in $\left|S_{1}-S_{2}\right|$ leads to a weak decrease in equilibrium total surplus. 
Summary of comparative statics results for the non-commitment case In this section we have found that there is at most one subgame perfect Nash equilibrium for each combination of $S_{i}, S_{j}$ and $\delta$. In contrast to the commitment situation, in the case without commitment it is possible that the equilibrium price decreases over time and that a firm's profit increases when it produces less. Increasing disparity in firm size leads to higher first-period equilibrium prices and lower sales, but has ambiguous effects on secondperiod equilibrium prices. Within every equilibrium outcome region, an increase in some firm's production level leads to an increase in it's profit, an increase in consumer surplus and an increase in total surplus. However, there are situations in which an increase in some firm's production level can lead to a decrease in its profits, a decrease in consumer surplus and/or a decrease in total surplus.

\subsection{Commitment versus Non-commitment}

In this section, we analyze how the equilibrium outcomes of the commitment setting are related to the equilibrium outcomes of the non-commitment case.

For certain regions, as was mentioned before, the equilibrium outcomes coincide. Notice that the equilibrium outcome corresponds to equilibrium sales by the two firms in both periods.

Proposition 2.5.1. For every $\left(S_{1}, S_{2}, \delta\right) \in \mathrm{X}_{l l}^{\mathrm{nc}} \cup \mathrm{X}_{l m}^{\mathrm{nc}} \cup \mathrm{X}_{m l}^{\mathrm{nc}} \cup \mathrm{X}_{l h}^{\mathrm{nc}} \cup \mathrm{X}_{h l}^{\mathrm{nc}} \cup \mathrm{X}_{m m}^{\mathrm{nc}} \cup$ $\mathrm{X}_{h h}^{\mathrm{nc}}$, the equilibrium sales in the non-commitment case coincide with those of the commitment setting.

Proof From the constraints defining the various regions it follows that $\mathrm{X}_{\mathrm{ll}}^{\mathrm{nc}} \subseteq \mathrm{X}_{\mathrm{ll}}^{\mathrm{c}}, \mathrm{X}_{\mathrm{lm}}^{\mathrm{nc}} \subseteq \mathrm{X}_{\mathrm{lm}}^{\mathrm{c}}, \mathrm{X}_{\mathrm{ml}}^{\mathrm{nc}} \subseteq \mathrm{X}_{\mathrm{ml}}^{\mathrm{c}}, \mathrm{X}_{\mathrm{lh}}^{\mathrm{nc}} \subseteq \mathrm{X}_{\mathrm{lh}}^{\mathrm{c}}, \mathrm{X}_{\mathrm{hl}}^{\mathrm{nc}} \subseteq \mathrm{X}_{\mathrm{hl}}^{\mathrm{c}}, \mathrm{X}_{\mathrm{mm}}^{\mathrm{nc}} \subseteq \mathrm{X}_{\mathrm{mm}}^{\mathrm{c}}$, and $\mathrm{X}_{\mathrm{hh}}^{\mathrm{nc}} \subseteq$ $\mathrm{X}_{\mathrm{hh}}^{\mathrm{c}}$. The equilibrium sales in Regions $\mathrm{X}_{\mathrm{ll}}^{\mathrm{nc}}, \mathrm{X}_{\mathrm{lm}}^{\mathrm{nc}}, \mathrm{X}_{\mathrm{ml}}^{\mathrm{nc}}, \mathrm{X}_{\mathrm{lh}}^{\mathrm{nc}}, \mathrm{X}_{\mathrm{hl}}^{\mathrm{nc}}, \mathrm{X}_{\mathrm{mm}}^{\mathrm{nc}}$, and $\mathrm{X}_{\mathrm{hh}}^{\mathrm{nc}}$ coincide with the equilibrium sales in Region $\mathrm{X}_{\mathrm{ll}}^{\mathrm{c}}, \mathrm{X}_{\mathrm{lm}}^{\mathrm{c}}, \mathrm{X}_{\mathrm{ml}}^{\mathrm{c}}, \mathrm{X}_{\mathrm{lh}}^{\mathrm{c}}, \mathrm{X}_{\mathrm{hl}}^{\mathrm{c}}, \mathrm{X}_{\mathrm{mm}}^{\mathrm{c}}$, and $\mathrm{X}_{\mathrm{hh}}^{\mathrm{c}}$, respectively.

An equilibrium always exists when $S_{1}=S_{2}$. Since, for these production levels, $\left(S_{1}, S_{1}, \delta\right) \in \mathrm{X}_{1 \mathrm{l}}^{\mathrm{nc}} \cup \mathrm{X}_{\mathrm{mm}}^{\mathrm{nc}} \cup \mathrm{X}_{\mathrm{hh}}^{\mathrm{nc}}$ when there is no commitment and $\left(S_{1}, S_{1}, \delta\right) \in \mathrm{X}_{\mathrm{ll}}^{\mathrm{c}} \cup \mathrm{X}_{\mathrm{mm}}^{\mathrm{c}} \cup \mathrm{X}_{\mathrm{hh}}^{\mathrm{c}}$ when there is commitment, the following corollary follows.

Corollary 2.5.2. When firms 1 and 2 are symmetric, the equilibrium sales in the commitment case coincide with those of the non-commitment setting. 
The equivalence in equilibrium outcomes no longer holds when $\left(S_{i}, S_{j}, \delta\right) \in$ $\mathrm{X}_{\mathrm{mh}}^{\mathrm{nc}} \cup \mathrm{X}_{\mathrm{hm}}^{\mathrm{nc}} \cup \mathrm{X}_{\mathrm{m}^{\prime} \mathrm{h}}^{\mathrm{nc}} \cup \mathrm{X}_{\mathrm{hm}^{\prime}}^{\mathrm{nc}} \cup \mathrm{X}_{\varnothing}^{n c}$. In these cases, there is one firm of intermediate size, and one firm that can react almost unrestrictedly to the quantities of its competitor. In the following we refer to these firms as the intermediate firm and the large firm, respectively. We show that the large firm gains and the intermediate firm loses from being in the non-commitment case, whenever we are not in Region $\mathrm{X}_{\varnothing}^{n c}$, i.e. whenever a subgame perfect Nash equilibrium exists in the non-commitment case.

Proposition 2.5.3. For every $\left(S_{1}, S_{2}, \delta\right)$ outside Region $\mathrm{X}_{\varnothing}^{n c}$, the change in equilibrium outcome from the commitment case to the non-commitment case is to the advantage of the larger firm and to the disadvantage of the smaller firm.

Proof Assume without loss of generality that $S_{2} \geq S_{1}$. Whenever there is a change in the equilibrium outcome, it holds that $\left(S_{1}, S_{2}, \delta\right)$ belongs to Region $\mathrm{X}_{\mathrm{mh}}^{\mathrm{c}}$. Firm 2 has profits equal to

$$
\Pi_{2}^{\mathrm{c}}=\left(\frac{2+4 \delta-3 \delta S_{1}}{6+6 \delta}\right)^{2}+\delta\left(\frac{4+2 \delta-3 S_{1}}{6+6 \delta}\right)^{2} .
$$

It also holds that $\left(S_{1}, S_{2}, \delta\right)$ belongs to Region $\mathrm{X}_{\mathrm{mh}}^{\mathrm{nc}}$ or Region $\mathrm{X}_{\mathrm{m}^{\prime} \mathrm{h}}^{\mathrm{nc}}$. In Region $\mathrm{X}_{\mathrm{mh}}^{\mathrm{nc}}$, firm 2 has profits equal to

$$
\Pi_{2}^{\mathrm{nc}}=\left(\frac{2+3 \delta-2 \delta S_{1}}{6+4 \delta}\right)^{2}+\delta\left(\frac{4+\delta-3 S_{1}}{6+4 \delta}\right)^{2} .
$$

In Region $\mathrm{X}_{\mathrm{m}^{\prime} \mathrm{h}}^{\mathrm{nc}}$ the profits of firm 2 are equal to

$$
\Pi_{2}^{\mathrm{nc}}=\frac{4}{9}+\frac{1}{9} \delta-\frac{2}{3} S_{1}+\frac{1}{4}\left(S_{1}\right)^{2} .
$$

We have that $S_{1} \leq \frac{2}{3}$ in all these regions, from which it follows that $\Pi_{2}^{\mathrm{nc}} \geq \Pi_{2}^{\mathrm{c}}$.

Analogous calculations show the opposite relation for the profits of firm 1 .

The intuition for this proposition follows from the same line of reasoning as that of Proposition 2.4.4. The total quantity sold is, in both the settings, the same for each firm. The small firm sells all of its stock in two periods, whereas the large firm reacts per-period optimal. The small firm sells more of its stock in the first period commitment setting than in the first period non-commitment setting and for the large firm it is the other way around. 
Price is higher in the first period non-commitment setting than in the first period commitment setting (see Proposition 2.5.4). For the second period, it is the other way around again. Therefore, the large firm makes more profit and the small firm makes less profit in the non-commitment setting, compared to the commitment setting. The small firm cannot change this by selling more of its stock in the first period, since this will induce the large firm to sell extra in the second period, thereby making this deviation unprofitable.

The following proposition describes the consequences of commitment for equilibrium prices and sales.

Proposition 2.5.4. For every $\left(S_{1}, S_{2}, \delta\right)$ outside Region $\mathrm{X}_{\varnothing}^{\mathrm{nc}}$, the first-period equilibrium price in the non-commitment case is greater than or equal to the first-period equilibrium price in the commitment case and the secondperiod equilibrium price in the non-commitment case is less than or equal to the second-period equilibrium price in the commitment setting. The opposite relationships hold for aggregate sales in the two periods.

Proof We assume without loss of generality that $S_{2} \geq S_{1}$. Whenever there is a change in the equilibrium price, $\left(S_{1}, S_{2}, \delta\right)$ belongs to Region $\mathrm{X}_{\mathrm{mh}}^{\mathrm{c}}$. In Region $\mathrm{X}_{\mathrm{mh}}^{\mathrm{c}}$, prices in the first and second period are respectively

$$
p_{1}^{\mathrm{c}}=\frac{2+4 \delta-3 \delta S_{1}}{6+6 \delta} \text { and } p_{2}^{\mathrm{c}}=\frac{4+2 \delta-3 S_{1}}{6+6 \delta} .
$$

It also holds that $\left(S_{1}, S_{2}, \delta\right)$ belongs to Region $\mathrm{X}_{\mathrm{mh}}^{\mathrm{nc}}$ or $\mathrm{X}_{\mathrm{m}^{\prime} \mathrm{h}}^{\mathrm{nc}}$. In Region $\mathrm{X}_{\mathrm{mh}}^{\mathrm{nc}}$, prices are

$$
p_{1}^{\mathrm{nc}}=\frac{2+3 \delta-2 \delta S_{1}}{6+4 \delta} \text { and } p_{2}^{\mathrm{nc}}=\frac{4+\delta-3 S_{1}}{6+4 \delta}
$$

and in Region $\mathrm{X}_{\mathrm{m}^{\prime} \mathrm{h}}^{\mathrm{nc}}$, prices are

$$
p_{1}^{\mathrm{nc}}=\frac{2}{3}-\frac{1}{2} S_{1} \text { and } p_{2}^{\mathrm{nc}}=\frac{1}{3} .
$$

In Region $\mathrm{X}_{\mathrm{mh}}^{\mathrm{c}}$ it holds that $(1-\delta) / 3<S_{1} \leq \frac{2}{3}$, from which it follows that $p_{1}^{\mathrm{c}} \leq p_{1}^{\mathrm{nc}}$ and $p_{2}^{\mathrm{c}} \geq p_{2}^{\mathrm{nc}}$.

The equilibrium outcome in the commitment case does not always coincide with the equilibrium outcome in the non-commitment setting, in particular when there is one intermediate and one large firm. In these cases, 
it is the intermediate firm that would deviate if the commitment equilibrium quantities were chosen in the non-commitment setting. By transferring some of its quantity from the first to the second period, the intermediate firm could improve its profit, knowing that it forces the bigger firm to adjust its second-period quantity downwards. This opportunity to deviate profitably leads to the non-existence of an equilibrium in Region $\mathrm{X}_{\varnothing}^{\mathrm{nc}}$.

In Regions $\mathrm{X}_{\mathrm{mh}}^{\mathrm{nc}}, \mathrm{X}_{\mathrm{hm}}^{\mathrm{nc}}, \mathrm{X}_{\mathrm{m}^{\prime} \mathrm{h}}^{\mathrm{nc}}$, and $\mathrm{X}_{\mathrm{hm}}^{\mathrm{nc}}$, the profitable deviation of the intermediate firm results in a change in the equilibrium outcome. Perhaps surprisingly, the equilibrium outcomes change to the disadvantage of the intermediate firm. To avoid a deviation by the intermediate firm, in the noncommitment case the large firm sets a higher first-period quantity than in the commitment case. This increase in sales by the large firm is more than offset by lower first-period sales by the intermediate firm. The first-period equilibrium price is higher in the non-commitment case than in the commitment setting. The intermediate firm still sells all its production, leading to a strong increase in its second-period sales. The second-period equilibrium price is lower in the non-commitment case than in the commitment setting. The large firm reacts per-period optimal to the intermediate firms and has the same total sales as before. It follows that the profit for the intermediate firm is lower in the non-commitment setting than in the commitment setting, whilst it is the other way around for the large firm.

Regarding consumer surplus, we mention the following. One may expect the ability to commit to lead to less competition in the commitment setting than in the case without commitment. However, this only holds for some settings in which future profits are hardly discounted. The non-commitment setting gives the large firm more opportunity to use its power, which, as a result, increases the first period price and decreases the second period price. Due to discounting, in most cases this results in consumer surplus being lower in the non-commitment setting than in the case with commitment.

Proposition 2.5.5. For every $\left(S_{1}, S_{2}, \delta\right)$, such that $\left(S_{1}, S_{2}, \delta\right) \notin \mathrm{X}_{\emptyset}^{\mathrm{nc}}$ and $\delta \leq$ $\frac{24}{25}$, consumers prefer the commitment setting over the non-commitment setting. If $\delta>\frac{24}{25}$, there are combinations of $\left(S_{1}, S_{2}, \delta\right)$ for which consumers prefer the non-commitment setting.

Proof We assume, without loss of generality, that $S_{2} \geq S_{1}$. Whenever there is a change in consumer surplus between the settings, $\left(S_{1}, S_{2}, \delta\right)$ belongs to Region $\mathrm{X}_{\mathrm{mh}}^{\mathrm{c}}$, and to Region $\mathrm{X}_{\mathrm{mh}}^{\mathrm{nc}}$ or $\mathrm{X}_{\mathrm{m}^{\prime} \mathrm{h}}^{\mathrm{nc}}$. Consumer surplus in Regions $\mathrm{X}_{\mathrm{mh}}^{\mathrm{c}}$, 
$\mathrm{X}_{\mathrm{mh}}^{\mathrm{nc}}$ and $\mathrm{X}_{\mathrm{m}{ }^{\prime} \mathrm{h}}^{\mathrm{nc}}$ is respectively

$$
\begin{aligned}
\mathrm{CS}_{\mathrm{mh}}^{\mathrm{c}} & =\left(\frac{4+2 \delta+3 \delta S_{1}}{6+6 \delta}\right)^{2}+\delta\left(\frac{3 S_{1}+2+4 \delta}{6+6 \delta}\right)^{2} \\
\mathrm{CS}_{\mathrm{mh}}^{\mathrm{nc}} & =\left(\frac{4+\delta+2 \delta S_{1}}{6+4 \delta}\right)^{2}+\delta\left(\frac{3 S_{1}+2+3 \delta}{6+4 \delta}\right)^{2} \\
\mathrm{CS}_{\mathrm{m}^{\prime} \mathrm{h}}^{\mathrm{nc}} & =\left(\frac{1}{2} S_{1}+\frac{1}{3}\right)^{2}+\frac{4}{9} \delta .
\end{aligned}
$$

For Region $\mathrm{X}_{\mathrm{mh}}^{\mathrm{nc}}$ and $\mathrm{X}_{\mathrm{m}^{\prime} \mathrm{h}}^{\mathrm{nc}}$, it holds respectively that $\frac{1}{3}(1-\delta) \leq S_{i} \leq \frac{2}{3}-\frac{1}{9} \delta$ and $\frac{2}{3}-\frac{1}{9} \delta<S_{i} \leq \frac{2}{3}$. Now,

$$
\mathrm{CS}_{\mathrm{mh}}^{\mathrm{c}} \geq \mathrm{CS}_{\mathrm{mh}}^{\mathrm{nc}} \text { for } S_{i} \in\left[\frac{1}{3}(1-\delta), \frac{24-7 \delta-17 \delta^{2}}{3 \delta}\right]
$$

and

$$
\mathrm{CS}_{\mathrm{mh}}^{\mathrm{c}} \geq \mathrm{CS}_{\mathrm{m}^{\prime} \mathrm{h}}^{\mathrm{nc}} \text { for } S_{i} \in\left[-2+\frac{8}{3} \delta, \frac{2}{3}\right]
$$

It holds that

$$
\frac{24-7 \delta-17 \delta^{2}}{3 \delta} \geq \frac{2}{3}-\frac{1}{9} \delta
$$

iff $\delta \leq \frac{24}{25}$ and

$$
-2+\frac{8}{3} \delta \leq \frac{2}{3}-\frac{1}{9} \delta
$$

iff $\delta \leq \frac{24}{25}$. So for $\delta \leq \frac{24}{25}$, consumers prefer the commitment setting over the non-commitment setting.

The smaller firm prefers the commitment setting, the larger firm prefers the non-commitment setting and consumers prefer in most situations the commitment setting. Which setting then maximizes total surplus is the question yet to answer.

Proposition 2.5.6. Total surplus is higher in the commitment setting than in the non-commitment setting.

Proof We again assume w.l.o.g. that $S_{2} \geq S_{1}$. Whenever there is a change in total surplus between the commitment and the non-commitment setting, $\left(S_{1}, S_{2}, \delta\right)$ belongs to Region $\mathrm{X}_{\mathrm{mh}}^{\mathrm{c}}$, and to Region $\mathrm{X}_{\mathrm{mh}}^{\mathrm{nc}}$ or $\mathrm{X}_{\mathrm{m}{ }^{\prime} \mathrm{h}}^{\mathrm{nc}}$. Total surplus 
in Regions $\mathrm{X}_{\mathrm{mh}}^{\mathrm{c}}, \mathrm{X}_{\mathrm{mh}}^{\mathrm{nc}}$ and $\mathrm{X}_{\mathrm{m}{ }^{\mathrm{h}}}^{\mathrm{nc}}$ is respectively

$$
\begin{aligned}
\mathrm{TS}_{\mathrm{mh}}^{\mathrm{c}} & =\left(\frac{4+2 \delta+3 \delta S_{1}}{6+6 \delta}\right)\left(1-\frac{1}{2}\left(\frac{4+2 \delta+3 \delta S_{1}}{6+6 \delta}\right)\right)+\delta\left(\frac{3 S_{1}+2+4 \delta}{6+6 \delta}\right)\left(1-\frac{1}{2}\left(\frac{3 S_{1}+2+4 \delta}{6+6 \delta}\right)\right), \\
\mathrm{TS}_{\mathrm{mh}}^{\mathrm{nc}} & =\left(\frac{4+\delta+2 \delta S_{1}}{6+4 \delta}\right)\left(1-\frac{1}{2}\left(\frac{4+\delta+2 \delta S_{1}}{6+4 \delta}\right)\right)+\delta\left(\frac{3 S_{1}+2+3 \delta}{6+4 \delta}\right)\left(1-\frac{1}{2}\left(\frac{3 S_{1}+2+3 \delta}{6+4 \delta}\right)\right), \\
\mathrm{TS}_{\mathrm{m} \mathrm{m}^{\mathrm{h}}}^{\mathrm{nc}} & =\left(\frac{1}{2} S_{1}+\frac{1}{3}\right)\left(1-\frac{1}{2}\left(\frac{1}{2} S_{1}+\frac{1}{3}\right)\right)+\delta\left(\frac{2}{3}\right)\left(1-\frac{1}{3}\right) .
\end{aligned}
$$

For Region $\mathrm{X}_{\mathrm{hm}}^{\mathrm{nc}}$ and Region $\mathrm{X}_{\mathrm{hm}}^{\mathrm{nc}}$, it holds respectively that $\frac{1}{3}(1-\delta) \leq S_{i} \leq$ $\frac{2}{3}-\frac{1}{9} \delta$ and $\frac{2}{3}-\frac{1}{9} \delta<S_{i} \leq \frac{2}{3}$. Now,

$$
\mathrm{TS}_{\mathrm{mh}}^{\mathrm{nc}} \geq \mathrm{TS}_{\mathrm{mh}}^{\mathrm{c}} \text { iff } S_{1} \in\left[\frac{-12+5 \delta+7 \delta^{2}}{3 \delta}, \frac{1}{3}(1-\delta)\right]
$$

and

$$
\mathrm{TS}_{\mathrm{m} \mathrm{h}^{\mathrm{h}}}^{\mathrm{nc}} \geq \mathrm{TS}_{\mathrm{mh}}^{\mathrm{c}} \text { iff } S_{1} \in\left[\frac{2}{3}, 2-\frac{4}{3} \delta\right] .
$$

So, for $S_{1} \in\left[\frac{1}{3}(1-\delta), \frac{2}{3}\right]$, total surplus is the highest in the commitment setting.

Summary of comparative statics results for the commitment versus the non-commitment case

The following can be said about the equilibrium outcome regions. There is no difference between the equilibrium outcomes in the commitment and the non-commitment case if the firms are of equal size. Non-commitment is preferred over commitment only by the larger of the two firms. When there is no commitment, the first-period equilibrium price is higher and the second-period equilibrium price is lower than in the case with commitment. Consumer surplus is in most cases highest in the commitment setting and total surplus is in all cases highest in the commitment setting.

\subsection{Concluding Remarks}

We have shown that whether firms can or cannot commit to their sales strategy influences prices, sales quantities, profits and surplus. Comparative statics in the case with commitment conform to standard intuition. In the non-commitment situation, however, a number of counterintuitive results were found. First, equilibria may fail to exist. Moreover, in equilibrium prices may decrease over time and higher stocks can lead to lower revenues 
from sales. Large firms benefit from the absence of commitment, contrary to small firms and, in most cases, consumers.

We have limited the analysis to the case where competition takes place during two periods. We expect our main results to be true in the multiperiod setting as well, but we fail to have an analytically tractable model specification for that situation.

We have only analyzed the case where production has already taken place, and firms compete in sales strategies. An extension of the model could be to make the production capacity choice of the firms endogenous, if, again, the tractability issues can be overcome.

Another issue that should be addressed in future research is to what extent the choice for quantity competition affects our outcomes. It is natural to address the questions of this chapter for models of price competition. Also here, however, it is challenging to find a model specification that is sufficiently general but still analytically tractable. 


\section{A The Reduced Best Response Correspondence}

We derive the reduced best response correspondence of firm $i$ for the noncommitment case. To keep the appendix within reasonable length, we have omitted the derivation of second-order conditions. In accordance with Proposition 2.4.1, we can restrict our analysis to best responses against $q_{j} \leq \frac{1}{2}$. We distinguish three cases:
(A) $q_{j}<S_{j}-\frac{1}{2}$
(B) $S_{j}-\frac{1}{2} \leq q_{j}<S_{j}-\frac{1}{3}$,
(C) $S_{j}-\frac{1}{3} \leq q_{j} \leq S_{j}$.

These three cases correspond to the three cases of residual stock $T_{j}=$ $S_{j}-q_{j}$ of firm $j$ with qualitatively different second-period behavior of firm $j$.
(A) $q_{j}<S_{j}-\frac{1}{2}$

Using the reduced profit function (2.2), for $0 \leq q_{i}<S_{i}-\frac{1}{3}$, profit is given by $\left(\mathrm{Y}_{\mathrm{hh}}\right)$, and for $S_{i}-\frac{1}{3} \leq q_{i} \leq S_{i}$, profit is given by $\left(\mathrm{Y}_{\mathrm{lh}}\right)$. Taking the unrestricted first-order condition of the profit function in $\left(\mathrm{Y}_{\mathrm{hh}}\right)$ and $\left(\mathrm{Y}_{\mathrm{lh}}\right)$ and solving for $q_{i}$ results in $q_{i}^{\text {hh }}$ and $q_{i}^{\text {lh }}$ given by

$$
\begin{aligned}
q_{i}^{\mathrm{hh}} & =\frac{1}{2}-\frac{1}{2} q_{j}, \\
q_{i}^{\mathrm{lh}} & =\frac{1-q_{j}-\frac{1}{2} \delta+\delta S_{i}}{2+\delta} .
\end{aligned}
$$

It holds that $q_{i}^{\text {hh }} \in\left[0, S_{i}-\frac{1}{3}\right)$ if and only if $\frac{5}{6}-\frac{1}{2} q_{j}<S_{i}$. Similarly, it holds that $q_{i}^{\mathrm{lh}} \in\left[S_{i}-\frac{1}{3}, S_{i}\right]$ if and only if $\frac{1}{2}-\frac{1}{2} q_{j}-\frac{1}{4} \delta \leq S_{i} \leq \frac{5}{6}-\frac{1}{12} \delta-\frac{1}{2} q_{j}$. We therefore find that the reduced best response $q_{i}^{*}$ of player 1 to $q_{j}$ is given by

$$
q_{i}^{*}= \begin{cases}S_{i}, & \text { if } 0 \leq S_{i}<\frac{1}{2}-\frac{1}{2} q_{j}-\frac{1}{4} \delta, \\ \frac{1-q_{j}-\frac{1}{2} \delta+\delta S_{i}}{2+\delta}, & \text { if } \frac{1}{2}-\frac{1}{2} q_{j}-\frac{1}{4} \delta \leq S_{i} \leq \frac{5}{6}-\frac{1}{12} \delta-\frac{1}{2} q_{j}, \\ S_{i}-\frac{1}{3}, & \text { if } \frac{5}{6}-\frac{1}{12} \delta-\frac{1}{2} q_{j}<S_{i} \leq \frac{5}{6}-\frac{1}{2} q_{j}, \\ \frac{1}{2}-\frac{1}{2} q_{j}, & \text { if } \frac{5}{6}-\frac{1}{2} q_{j}<S_{i} .\end{cases}
$$


(B) $S_{j}-\frac{1}{2} \leq q_{j}<S_{j}-\frac{1}{3}$

It follows from the reduced profit function (2.2) that, for $0 \leq q_{i}<S_{i}-\frac{1}{3}$, profit is given by $\left(\mathrm{Y}_{\mathrm{hh}}\right)$, for $S_{i}-\frac{1}{3} \leq q_{i}<2 T_{j}-1+S_{i}$, profit is given by ( $\left.\mathrm{Y}_{\mathrm{lh}}\right)$, and for $2 T_{j}-1+S_{i} \leq q_{i} \leq S_{i}$, profit is given by ( $\left.\mathrm{Y}_{11}\right)$. Taking the unrestricted first-order condition of the reduced profit function in $\left(\mathrm{Y}_{\mathrm{hh}}\right),\left(\mathrm{Y}_{\mathrm{lh}}\right)$ and $\left(\mathrm{Y}_{11}\right)$ and solving for $q_{i}$ results in $q_{i}^{\mathrm{hh}}, q_{i}^{\mathrm{lh}}$, and $q_{i}^{\mathrm{ll}}$ given by

$$
\begin{aligned}
q_{i}^{\mathrm{hh}} & =\frac{1}{2}-\frac{1}{2} q_{j}, \\
q_{i}^{\mathrm{lh}} & =\frac{1-q_{j}-\frac{1}{2} \delta+\delta S_{i}}{2+\delta}, \\
q_{i}^{\mathrm{ll}} & =\frac{1-q_{j}+2 \delta S_{i}-\delta+\delta T_{j}}{2+2 \delta} .
\end{aligned}
$$

It holds that $q_{i}^{\text {hh }} \in\left[0, S_{i}-\frac{1}{3}\right)$ if and only if $\frac{5}{6}-\frac{1}{2} q_{j}<S_{i}$. Similarly, it holds that $q_{i}^{\mathrm{lh}} \in\left[\max \left\{0, S_{i}-\frac{1}{3}\right\}, 2 T_{j}-1+S_{i}\right)$ if and only if $\underline{S}_{i}^{\mathrm{lh}}<S_{i} \leq \bar{S}_{i}^{\mathrm{lh}}$, where

$$
\begin{aligned}
\underline{S}_{i}^{\mathrm{lh}} & =\frac{3}{2}+\frac{1}{4} \delta-\frac{1}{2} q_{j}-(2+\delta) T_{j}, \\
\bar{S}_{i}^{\mathrm{lh}} & =\frac{5}{6}-\frac{1}{12} \delta-\frac{1}{2} q_{j} .
\end{aligned}
$$

The requirement $q_{i}^{\mathrm{lh}} \geq 0$ is not binding, since $q_{j} \leq \frac{1}{2}$ implies $q_{i}^{\mathrm{lh}}$ is positive. It holds that $q_{i}^{\mathrm{ll}} \in\left[\max \left\{0,2 T_{j}-1+S_{i}\right\}, S_{i}\right]$ if and only if $\max \left\{\underline{S}_{i}^{\mathrm{lla}}, \underline{S}_{i}^{\mathrm{llb}}\right\} \leq S_{i} \leq \bar{S}_{i}^{\mathrm{ll}}$, where

$$
\begin{aligned}
\underline{S}_{i}^{\mathrm{lla}} & =\frac{1}{2 \delta}\left(q_{j}-1+\delta-\delta T_{j}\right) \\
\underline{S}_{i}^{\mathrm{llb}} & =\frac{1}{2}\left(1-\delta-q_{j}+\delta T_{j}\right) \\
\bar{S}_{i}^{\mathrm{ll}} & =\frac{3}{2}+\frac{1}{2} \delta-\frac{1}{2} q_{j}-\left(2+\frac{3}{2} \delta\right) T_{j} .
\end{aligned}
$$

Since $S_{j}-q_{j} \geq 1 / 3$, it holds that $\max \left\{\bar{S}_{i}^{\mathrm{lh}}, \bar{S}_{i}^{\mathrm{ll}}\right\} \leq 5 / 6-q_{j} / 2$. The intervals $\left[\underline{S}_{i}^{\mathrm{lh}}, \bar{S}_{i}^{\mathrm{lh}}\right]$ and $\left[\max \left\{\underline{S}_{i}^{\mathrm{lla}}, \underline{S}_{i}^{\mathrm{llb}}\right\}, \bar{S}_{i}^{\mathrm{ll}}\right]$ are overlapping. In particular, since $q_{j} \leq$ $1 / 2, T_{j}=S_{j}-q_{j} \leq 1 / 2$, and $\delta \leq 1$, it holds that $\max \left\{\underline{S}_{i}^{\mathrm{lla}}, \underline{S}_{i}^{\mathrm{llb}}\right\} \leq \underline{S}_{i}^{\mathrm{lh}} \leq \bar{S}_{i}^{\mathrm{ll}}$.

The reduced profit function of firm $i$ has two local maxima if $\underline{S}_{i}^{\mathrm{lh}} \leq S_{i} \leq$ $\min \left\{\bar{S}_{i}^{\mathrm{ll}}, \bar{S}_{i}^{\mathrm{lh}}\right\}$. Since $\bar{S}_{i}^{\mathrm{lh}} \leq \bar{S}_{i}^{\mathrm{ll}}$ if and only if $q_{j} \geq S_{j}-\frac{8+7 \delta}{24+18 \delta}$, the profit function has two local maxima if

$$
S_{j}-\frac{1}{2} \leq q_{j} \leq S_{j}-\frac{8+7 \delta}{24+18 \delta} \text { and } \underline{S}_{i}^{\mathrm{lh}} \leq S_{i} \leq \bar{S}_{i}^{\mathrm{ll}}
$$


or

$$
S_{j}-\frac{8+7 \delta}{24+18 \delta} \leq q_{j} \leq S_{j}-\frac{1}{3} \text { and } \underline{S}_{i}^{\mathrm{lh}} \leq S_{i} \leq \bar{S}_{i}^{\mathrm{lh}} .
$$

To find the global maximum, we compare the profits in both local maxima. The profits corresponding to $q_{i}^{\mathrm{lh}}$ and $q_{i}^{\mathrm{ll}}$ are respectively

$$
\begin{aligned}
\Pi_{i}^{\mathrm{Mh}} & =\frac{4+4 q_{j}^{2}-4 \delta+16 \delta S_{i}+8 \delta S_{i}^{2}+\delta^{2}+-8 q_{j}+4 \delta q_{j}-8 S_{i} \delta q_{j}}{16+8 \delta}, \\
\Pi_{i}^{\mathrm{Il}} & =\frac{1-2 \delta+\delta^{2}-2 q_{j}+2 \delta^{2} q_{j}+q_{j}^{2}+2 \delta q_{j}^{2}+\delta^{2} q_{j}^{2}+8 \delta S_{i}-4 \delta S_{i}^{2}+2 \delta S_{j}-2 \delta^{2} S_{j}-2 \delta q_{j} S_{j}-2 \delta^{2} q_{j} S_{j}-4 \delta S_{i} S_{j}+\delta^{2} S_{j}^{2}}{4+4 \delta} .
\end{aligned}
$$

It holds that $\Pi_{i}^{\mathrm{lh}} \geq \Pi_{i}^{\mathrm{ll}}$ if and only if $S_{i} \geq \tilde{S}_{i}$, where

$$
\tilde{S}_{i}=1-\frac{1}{2} q_{j}-\left(1+\frac{1}{2} \delta\right) T_{j}+\left(\frac{1}{2}-T_{j}\right) \sqrt{(1+\delta)\left(1+\frac{1}{2} \delta\right)} .
$$

Since $\tilde{S}_{i}>\underline{S}_{i}^{\mathrm{lh}}$ whenever $q_{j} \geq S_{j}-\frac{1}{2}, q_{i}^{\mathrm{lh}}$ maximizes profits for $\tilde{S}_{i} \leq S_{i} \leq \bar{S}_{i}^{\mathrm{lh}}$.

Since $\max \left\{\underline{S}_{i}^{\mathrm{lla}}, \underline{S}_{i}^{\mathrm{llb}}\right\} \leq \tilde{S}_{i} \leq \bar{S}_{i}^{\mathrm{ll}}, q_{i}^{\mathrm{ll}}$ maximizes profits for $\max \left\{\underline{S}_{i}^{\mathrm{lla}}, \underline{S}_{i}^{\mathrm{llb}}\right\} \leq$ $S_{i} \leq \tilde{S}_{i}$

When $\max \left\{\bar{S}_{i}^{\mathrm{ll}}\left(q_{j}\right), \bar{S}_{i}^{\mathrm{lh}}\left(q_{j}\right)\right\}<S_{i} \leq \frac{5}{6}-\frac{1}{2} q_{j}$ we have a boundary solution, and profit maximizing sales are given by $q_{i}^{*}=S_{i}-\frac{1}{3}$.

One possibility remains: $\max \left\{\bar{S}_{i}^{\mathrm{lh}}, \tilde{S}_{i}\right\}<S_{i} \leq \bar{S}_{i}^{\mathrm{ll}}$. In this case, the profit maximizing choice is either $q_{i}^{\text {ll }}$ or $q_{i}^{*}$. We argue that $q_{i}^{\text {ll }}$ maximizes profits, so for $\bar{S}_{i}^{\mathrm{lh}} \leq S_{i} \leq \bar{S}_{i}^{\mathrm{ll}}$, the best response of firm $i$ is $q_{i}^{\mathrm{ll}}$.

It holds that

$$
\Pi_{i}^{\mathrm{ll}} \geq \Pi_{i}^{*}=\left(S_{i}-\frac{1}{3}\right)\left(\frac{4}{3}-S_{i}-q_{j}\right)+\frac{1}{9} \delta
$$

if and only if

$$
S_{i} \in\left[\frac{5}{6}-\frac{1}{6} \delta-\frac{1}{2} q_{j}+\frac{1}{2} \delta T_{j} \pm \frac{1}{3} \sqrt{\delta(1+\delta)\left(3 T_{j}-1\right)}\right] .
$$

Since

$$
\left[\bar{S}_{i}^{\mathrm{lh}}, \bar{S}_{i}^{\mathrm{ll}}\right] \subset\left[\frac{5}{6}-\frac{1}{6} \delta-\frac{1}{2} q_{j}+\frac{1}{2} \delta T_{j} \pm \frac{1}{3} \sqrt{\delta(1+\delta)\left(3 T_{j}-1\right)}\right]
$$

for

$$
q_{j}<S_{j}-\frac{4+5 \delta-4 \sqrt{(1+\delta)\left(1+\frac{1}{2} \delta\right)}}{6 \delta},
$$

we have our desired conclusion. 
Summarizing, the reduced best response $q_{i}^{*}$ of player $i$ against $q_{j}$ for $S_{j}-\frac{1}{2} \leq q_{j}<S_{j}-\frac{1}{3}$ is given by

$$
q_{i}^{*}= \begin{cases}0, & \text { if } 0 \leq S_{i}<\underline{S}_{i}^{\mathrm{lla}}, \\ S_{i}, & \text { if } 0 \leq S_{i}<\underline{S}_{i}^{\mathrm{llb}}, \\ \frac{\left(1-q_{j}+2 \delta S_{i}-\delta+\delta T_{j}\right)}{2+2 \delta}, & \text { if } \max \left\{\underline{S}_{i}^{\mathrm{lla}}, \underline{S}_{i}^{\mathrm{llb}}\right\} \leq S_{i} \leq \tilde{S}_{i}, \\ \frac{1-q_{j}-\frac{1}{2} \delta+\delta S_{i}}{2+\delta}, & \text { if } \tilde{S}_{i} \leq S_{i} \leq \bar{S}_{i}^{\mathrm{lh}}, \\ \frac{\left(1-q_{j}+2 \delta S_{i}-\delta+\delta T_{j}\right)}{2+2 \delta}, & \text { if } \max \left\{\tilde{S}_{i}, \bar{S}_{i}^{\mathrm{lh}}\right\}<S_{i} \leq \bar{S}_{i}^{\mathrm{ll}}, \\ S_{i}-\frac{1}{3} & \text { if } \max \left\{\bar{S}_{i}^{\mathrm{lh}}, \bar{S}_{i}^{1 \mathrm{ll}}\right\}<S_{i} \leq \frac{5}{6}-\frac{1}{2} q_{j}, \\ \frac{1}{2}-\frac{1}{2} q_{j}, & \text { if } \frac{5}{6}-\frac{1}{2} q_{j}<S_{i},\end{cases}
$$

where

$$
\begin{aligned}
\tilde{S}_{i} & =1-\frac{1}{2} q_{j}-\left(1+\frac{1}{2} \delta\right) T_{j}+\left(\frac{1}{2}-T_{j}\right) \sqrt{(1+\delta)\left(1+\frac{1}{2} \delta\right)}, \\
\underline{S}_{i}^{\mathrm{lh}} & =\frac{3}{2}+\frac{1}{4} \delta-\frac{1}{2} q_{j}-(2+\delta) T_{j}, \\
\bar{S}_{i}^{\mathrm{lh}} & =\frac{5}{6}-\frac{1}{12} \delta-\frac{1}{2} q_{j}, \\
\underline{S}_{i}^{\mathrm{lla}} & =\frac{1}{2 \delta}\left(q_{j}-1+\delta-\delta T_{j}\right), \\
\underline{S}_{i}^{\mathrm{llb}} & =\frac{1}{2}\left(1-\delta-q_{j}+\delta T_{j}\right), \\
\bar{S}_{i}^{\mathrm{ll}} & =\frac{3}{2}+\frac{1}{2} \delta-\frac{1}{2} q_{j}-\left(2+\frac{3}{2} \delta\right) T_{j} .
\end{aligned}
$$

(C) $S_{j}-\frac{1}{3} \leq q_{j} \leq S_{j}$

It follows from the reduced profit function (2.2) that, for $0 \leq q_{i}<S_{i}-\frac{1}{2}+\frac{1}{2} T_{j}$, profit is given by $\left(\mathrm{Y}_{\mathrm{hl}}\right)$, and for $S_{i}-\frac{1}{2}+\frac{1}{2} T_{j} \leq q_{i} \leq S_{i}$, profit is given by ( $\left.\mathrm{Y}_{\mathrm{ll}}\right)$. Taking the unrestricted first-order condition of the profit function in $\left(\mathrm{Y}_{\mathrm{hl}}\right)$ and $\left(\mathrm{Y}_{11}\right)$ and solving for $q_{i}$ results in $q_{i}^{\mathrm{hl}}$ and $q_{i}^{\mathrm{ll}}$ given by

$$
\begin{aligned}
q_{i}^{\mathrm{hl}} & =\frac{1}{2}-\frac{1}{2} q_{j}, \\
q_{i}^{\mathrm{ll}} & =\frac{1-q_{j}+2 \delta S_{i}-\delta+\delta T_{j}}{2+2 \delta} .
\end{aligned}
$$

It holds that $q_{i}^{\text {hl }} \in\left[0, S_{i}-\frac{1}{2}+\frac{1}{2} T_{j}\right)$ if and only if $1-\frac{1}{2} S_{j}<S_{i}$. Similarly, it holds that $q_{i}^{\mathrm{ll}} \in\left[S_{i}-\frac{1}{2}+\frac{1}{2} T_{j}, S_{i}\right]$ if and only if $\max \left\{\frac{1}{2}\left(1-\delta-q_{j}+\delta T_{j}\right), \frac{1}{2 \delta}(-1+\right.$ 
$\left.\left.\delta+q_{j}-\delta T_{j}\right)\right\} \leq S_{i} \leq 1-\frac{1}{2} S_{j}$. We therefore find that the reduced best response $q_{i}^{*}$ of player 1 to $q_{j}$ is given by

$$
q_{i}^{*}= \begin{cases}S_{i}, & \text { if } 0 \leq S_{i}<\frac{1}{2}\left(1-\delta-q_{j}+\delta T_{j}\right), \\ 0, & \text { if } 0 \leq S_{i}<\frac{1}{2 \delta}\left(-1+\delta+q_{j}-\delta T_{j}\right), \\ \frac{1-q_{j}+2 \delta S_{i}-\delta+\delta T_{j}}{2+2 \delta}, & \text { if } \max \left\{\frac{1}{2}\left(1-\delta-q_{j}+\delta T_{j}\right), \frac{1}{2 \delta}\left(-1+\delta+q_{j}-\delta T_{j}\right)\right\} \\ & \leq S_{i} \leq 1-\frac{1}{2} S_{j}, \\ \frac{1}{2}-\frac{1}{2} q_{j}, & \text { if } 1-\frac{1}{2} S_{j}<S_{i} .\end{cases}
$$

\section{Overview of the Reduced Best Response Correspondence}

Table 2.4 now follows immediately.

\section{B Subgame Perfect Equilibria}

We define the sets $A_{j}(1), \ldots, A_{j}(4), B_{j}(1), \ldots, B_{j}(7), C_{j}(1), \ldots, C_{j}(4)$ as the sets of quantities $q_{j}$ satisfying the constraints as presented in Table 2.4. Notice that each of these sets is a subset of [0,1/2]. Moreover, we define $A_{j}\left(k_{1}, \ldots, k_{\ell}\right)=$ $A_{j}\left(k_{1}\right) \cup \cdots \cup A_{j}\left(k_{\ell}\right)$, and similarly for sets $B_{j}\left(k_{1}, \ldots, k_{\ell}\right)$ and $C_{j}\left(k_{1}, \ldots, k_{\ell}\right)$. In the proofs we will make use of Table 2.4. That table presents the reduced best response of firm $i$ to a first-period sales quantity of firm $j$ with the use of coefficients $\alpha_{1}, \ldots, \alpha_{8}$. In the sequel we will need the reduced best response of firm $j$ to a first-period sales quantity of firm $i$, which follows from Table 2.4 by reversing the roles of firm $i$ and $j$. The corresponding coefficients are denoted by $\beta_{1}, \ldots, \beta_{8}$.

Proposition B.1 If $\left(q_{i}^{*}, q_{j}^{*}\right)$ is a Nash equilibrium of the reduced game and $q_{j}^{*} \in A_{j}(1,2,3) \cup B_{j}(1,2,3,4,5,6) \cup C_{j}(1)$, then $S_{i}-q_{i}^{*} \leq \frac{1}{3}$, so $q_{i}^{*} \in C_{i}(1,2,3,4)$.

Proof For $q_{j}^{*} \in A_{j}(1,3) \cup B_{j}(1,6) \cup C_{j}(1)$ it follows immediately from Table 2.4 that $S_{i}-q_{i}^{*} \leq \frac{1}{3}$. For $q_{j}^{*} \in A_{j}(2) \cup B_{j}(4)$,

$$
S_{i}-q_{i}^{*}=\frac{2 S_{i}-1+q_{j}^{*}+\frac{1}{2} \delta}{2+\delta} \leq \frac{\frac{2}{3}+\frac{1}{3} \delta}{2+\delta}=\frac{1}{3},
$$

where the inequality follows from $q_{j}^{*} \leq \alpha_{2}$. For $q_{j}^{*} \in B_{j}(2)$,

$$
S_{i}-q_{i}^{*}<\frac{3}{4}-\frac{1}{4 \delta}-\frac{1}{2} S_{j}<\frac{1}{3}
$$




\begin{tabular}{l||l|l}
$A_{j}\left(q_{j}<S_{j}-\frac{1}{2}\right)$ & $q_{i}^{*}$ & $r_{i}^{*}$ \\
\hline \hline 1) $0 \leq q_{j}<\alpha_{1}$ & $S_{i}$ & 0 \\
\hline 2) $\alpha_{1} \leq q_{j} \leq \alpha_{2}$ & $\frac{1-q_{j}-\frac{1}{2} \delta+\delta S_{i}}{2+\delta}$ & $\frac{2 S_{i}-1+q_{j}+\frac{1}{2} \delta}{2+\delta}$ \\
\hline 3) $\alpha_{2}<q_{j} \leq \alpha_{3}$ & $S_{i}-\frac{1}{3}$ & $\frac{1}{3}$ \\
\hline 4) $q_{j}>\alpha_{3}$ & $\frac{1}{2}-\frac{1}{2} q_{j}$ & $\frac{1}{3}$
\end{tabular}

\begin{tabular}{l||l|l}
$B_{j}\left(S_{j}-\frac{1}{2} \leq q_{j}<S_{j}-\frac{1}{3}\right)$ & $q_{i}^{*}$ & $r_{i}^{*}$ \\
\hline \hline 1) $0 \leq q_{j}<\alpha_{4}$ & $S_{i}$ & 0 \\
\hline 2) $q_{j}>\alpha_{5}$ & 0 & $S_{i}$ \\
\hline 3) $\max \left\{\alpha_{4}, \alpha_{6}\right\} \leq q_{j} \leq \alpha_{5}$ & $\frac{1-q_{j}+2 \delta S_{i}-\delta+\delta T_{j}}{2+2 \delta}$ & $\frac{2 S_{i}-1+q_{j}+\delta-\delta T_{j}}{2+2 \delta}$ \\
\hline 4) $q_{j} \leq \alpha_{6}, q_{j} \leq \alpha_{2}$ & $\frac{1-q_{j}-\frac{1}{2} \delta+\delta S_{i}}{2+\delta}$ & $\frac{2 S_{i}-1+q_{j}+\frac{1}{2} \delta}{2+\delta}$ \\
\hline 5) $\alpha_{2}<q_{j}<\alpha_{6}, q_{j} \geq \alpha_{7}$ & $\frac{1-q_{j}+2 \delta S_{i}-\delta+\delta T_{j}}{2+2 \delta}$ & $\frac{2 S_{i}-1+q_{j}+\delta-\delta T_{j}}{2+2 \delta}$ \\
\hline 6) $\alpha_{2}<q_{j} \leq \alpha_{3}, q_{j}<\alpha_{7}$ & $S_{i}-\frac{1}{3}$ & $\frac{1}{3}$ \\
\hline 7) $q_{j}>\alpha_{3}$ & $\frac{1}{2}-\frac{1}{2} q_{j}$ & $\frac{1}{3}$
\end{tabular}

\begin{tabular}{l||l|l}
$C_{j}\left(q_{j} \geq S_{j}-\frac{1}{3}\right)$ & $q_{i}^{*}$ & $r_{i}^{*}$ \\
\hline \hline 1) $0 \leq q_{j}<\alpha_{4}$ & $S_{i}$ & 0 \\
\hline 2) $q_{j}>\alpha_{5}$ & 0 & $S_{i}$ \\
\hline 3) $\alpha_{4} \leq q_{j} \leq \alpha_{5}, S_{i} \leq \alpha_{8}$ & $\frac{1-q_{j}+2 \delta S_{i}-\delta+\delta T_{j}}{2+2 \delta}$ & $\frac{2 S_{i}-1+q_{j}+\delta-\delta T_{j}}{2+2 \delta}$ \\
\hline 4) $S_{i}>\alpha_{8}$ & $\frac{1}{2}-\frac{1}{2} q_{j}$ & $\frac{1}{2}-\frac{1}{2} S_{j}+\frac{1}{2} q_{j}$
\end{tabular}

\begin{tabular}{l|l} 
& Explanation of the symbols \\
\hline$\alpha_{1}$ & $1-\frac{1}{2} \delta-2 S_{i}$ \\
$\alpha_{2}$ & $\frac{5}{3}-\frac{1}{6} \delta-2 S_{i}$ \\
$\alpha_{3}$ & $\frac{5}{3}-2 S_{i}$ \\
$\alpha_{4}$ & $\frac{1-\delta+\delta S_{j}-2 S_{i}}{1+\delta}$ \\
$\alpha_{5}$ & $\frac{1-\delta+\delta S_{j}+2 \delta S_{i}}{1+\delta}$ \\
$\alpha_{6}$ & $\frac{2 S_{i}-2+2 S_{j}+\delta S_{j}-\left(1-2 S_{j}\right) \sqrt{(1+\delta)\left(1+\frac{1}{2} \delta\right)}}{1+\delta+2 \sqrt{(1+\delta)\left(1+\frac{1}{2} \delta\right)}}$ \\
$\alpha_{7}$ & $\frac{2 S_{i}-3-\delta+4 S_{j}+3 \delta S_{j}}{3+3 \delta}$ \\
$\alpha_{8}$ & $1-\frac{1}{2} S_{j}$
\end{tabular}

Table 2.4: Reduced best response correspondence $\sigma_{i}^{\mathrm{R} *}\left(q_{j}\right)$ for $0 \leq q_{j} \leq \frac{1}{2}$. 
where the first inequality follows from $\alpha_{5}<q_{j}^{*} \leq \frac{1}{2}$ and the second one from $\delta \leq 1$ and $S_{j}>q_{j}^{*}+\frac{1}{3} \geq \frac{1}{3}$. For $q_{j}^{*} \in B_{j}(3)$,

$$
\begin{aligned}
S_{i}-q_{i}^{*} & =\frac{2 S_{i}-1+q_{j}^{*}+\delta-\delta T_{j}}{2(1+\delta)} \leq \frac{\left(1+\delta+\sqrt{(1+\delta)\left(1+\frac{1}{2} \delta\right)}\right)\left(1-2 T_{j}\right)}{2(1+\delta)} \\
& \leq \frac{1+\delta+\sqrt{(1+\delta)\left(1+\frac{1}{2} \delta\right)}}{6(1+\delta)} \leq \frac{1}{3},
\end{aligned}
$$

where the first inequality follows from $q_{j}^{*} \geq \alpha_{6}$ (i.e. $S_{i} \leq S_{i}^{\mathrm{c}}$ ), the second from $\frac{1}{3}<T_{j} \leq \frac{1}{2}$ and the third one from $\delta \in(0,1]$. For $q_{j}^{*} \in B_{j}(5)$,

$$
S_{i}-q_{i}^{*}=\frac{2 S_{i}-1+q_{j}^{*}+\delta-\delta T_{j}}{2(1+\delta)} \leq 1-2 T_{j} \leq \frac{1}{3},
$$

where the first inequality follows from $q_{j}^{*} \leq \alpha_{7}$, i.e. $S_{i} \leq \bar{S}_{i}^{\mathrm{ll}}$, and the second one from $\frac{1}{3}<T_{j} \leq \frac{1}{2}$.

Proposition B.2 If $\left(q_{i}^{*}, q_{j}^{*}\right)$ is a Nash equilibrium of the reduced game and $q_{j}^{*} \in A_{j}(4) \cup B_{j}(7) \cup C_{j}(4)$, then $S_{i}-q_{i}^{*}>\frac{1}{3}$, so $q_{i}^{*} \in A_{i}(1,2,3,4) \cup B_{i}(1,2,3,4,5,6,7)$.

Proof If $q_{j}^{*} \in A_{j}(4) \cup B_{j}(7)$, then since $q_{j}^{*}>\alpha_{3}$, we have $S_{i}>\frac{5}{6}-\frac{1}{2} q_{j}^{*}$, and $q_{i}^{*}=\frac{1}{2}-\frac{1}{2} q_{j}^{*}$. Therefore, $S_{i}-q_{i}^{*}>\frac{1}{3}$. If $q_{j}^{*} \in C_{j}(4)$, then $S_{j}-q_{j}^{*} \leq \frac{1}{3}, S_{i}>$ $1-\frac{1}{2} S_{j}$, and $q_{i}^{*}=\frac{1}{2}-\frac{1}{2} q_{j}^{*}$. This implies $S_{i}-q_{i}^{*}>\frac{1}{3}$.

We continue by solving for all Nash equilibria $\left(q_{i}^{*}, q_{j}^{*}\right)$ of the reduced game where $q_{j}^{*} \in A_{j}(1)$. Next we consider Nash equilibria $\left(q_{i}^{*}, q_{j}^{*}\right)$ with $q_{j}^{*} \in A_{j}(2)$. We restrict attention to the case with $q_{i}^{*} \notin A_{i}(1)$, since using the symmetry of the firms such equilibria follow already from the first case. We continue with $q_{j}^{*} \in A_{j}(3)$, and so on. 
$\mathbf{q}_{\mathbf{j}}^{*} \in \mathbf{A}_{\mathbf{j}}(\mathbf{1})$

It holds that

$$
\begin{aligned}
& q_{j}^{*}<S_{j}-\frac{1}{2}, \\
& q_{j}^{*}<1-\frac{1}{2} \delta-2 S_{i}, \\
& q_{i}^{*}=\sigma_{i}^{\mathrm{R}}\left(q_{j}^{*}\right)=S_{i} .
\end{aligned}
$$

By Proposition B.1, $q_{i}^{*} \in C_{i}(1,2,3,4)$. This gives the following possibilities:

$$
\begin{array}{ll}
q_{i}^{*} \in C_{i}(1): & q_{j}^{*}=S_{j}, \\
q_{i}^{*} \in C_{i}(2): & q_{j}^{*}=0, \\
q_{i}^{*} \in C_{i}(3): & q_{j}^{*}=\frac{1-S_{i}+2 \delta S_{j}-\delta}{2+2 \delta}, \\
q_{i}^{*} \in C_{i}(4): & q_{j}^{*}=\frac{1}{2}-\frac{1}{2} S_{i} .
\end{array}
$$

If $q_{i}^{*} \in C_{i}(2)$, then $q_{i}^{*}>\beta_{5}$ implies $S_{j}<\frac{1}{2}-\frac{1}{2 \delta}+\frac{1}{2 \delta} S_{i}<\frac{1}{2}$ by (2.5) and Lemma 2.4.1, so (2.3) leads to a contradiction.

Next, (2.3) and (2.6) imply $S_{j}>1-\frac{1}{2} S_{i}$, whereas $q_{i}^{*} \in C_{i}(3)$ implies $q_{i}^{*} \leq$ $\beta_{8}$, so $S_{j} \leq 1-\frac{1}{2} S_{i}$, a contradiction.

When $q_{i}^{*} \in C_{i}(4)$, then $q_{i}^{*} \geq S_{i}-\frac{1}{3}$ and $S_{j}>\beta_{8}$. These inequalities together with the inequalities (2.3) and (2.4) lead to the conclusion that $\left(q_{j}^{*}, q_{i}^{*}\right)$ is a Nash equilibrium with $q_{j}^{*} \in A_{j}(1)$ if and only if $q_{j}^{*}=\frac{1}{2}-\frac{1}{2} S_{i}, q_{i}^{*}=S_{i}$, $S_{j}>1-\frac{1}{2} S_{i}$, and $S_{i}<\frac{1}{3}-\frac{1}{3} \delta$.

$$
\mathbf{q}_{\mathbf{j}}^{*} \in \mathbf{A}_{\mathbf{j}}(\mathbf{2})
$$

It holds that

$$
\begin{aligned}
& q_{j}^{*}<S_{j}-\frac{1}{2}, \\
& 1-\frac{1}{2} \delta-2 S_{i} \leq q_{j}^{*} \leq \frac{5}{3}-\frac{1}{6} \delta-2 S_{i}, \\
& q_{i}^{*}=\sigma_{i}^{\mathrm{R}}\left(q_{j}^{*}\right)=\frac{1-q_{j}^{*}-\frac{1}{2} \delta+\delta S_{i}}{2+\delta} \leq \frac{1}{2} .
\end{aligned}
$$


By Proposition B.1, $q_{i}^{*} \in C_{i}(2,3,4){ }^{7}$ This gives the following possibilities:

$$
\begin{aligned}
q_{i}^{*} \in C_{i}(2): q_{i}^{*} & =\frac{1-\frac{1}{2} \delta+\delta S_{i}}{2+\delta}, q_{j}^{*}=0 \\
q_{i}^{*} \in C_{i}(3): q_{i}^{*} & =\frac{1+2 \delta-\delta^{2}+\delta S_{i}+2 \delta^{2} S_{i}-2 \delta S_{j}}{3+5 \delta+2 \delta^{2}} \\
q_{j}^{*} & =\frac{2-3 \delta-\delta^{2}+8 \delta S_{j}+4 \delta^{2} S_{j}+2 \delta S_{i}}{6+10 \delta+4 \delta^{2}}, \\
q_{i}^{*} \in C_{i}(4): q_{i}^{*} & =\frac{1-\delta+2 \delta S_{i}}{3+2 \delta}, q_{j}^{*}=\frac{2+3 \delta-2 \delta S_{i}}{6+4 \delta} .
\end{aligned}
$$

Consider $q_{i}^{*} \in C_{i}(2)$. Then $q_{i}^{*}>\beta_{5}$, so $S_{j}<\frac{-2+3 \delta+\delta^{2}-2 \delta S_{i}}{8 \delta+4 \delta^{2}}<\frac{1}{2}$, and (2.8) leads to a contradiction.

Consider $q_{i}^{*} \in C_{i}(3)$. It holds that

$$
\frac{5+2 \delta+\delta^{2}+2 \delta S_{i}}{6+2 \delta}<S_{j} \leq 1-\frac{1}{2} S_{i}
$$

where the first inequality follows from (2.8) and (2.11), and the second inequality from $S_{j} \leq \beta_{8}$. By rewriting the expression in (2.13), it follows that $S_{i}<\frac{1}{3}-\frac{1}{3} \delta$.

However, this is contradicted by

$$
S_{i} \geq \frac{1+2 \delta-\delta^{2}-2 \delta S_{j}}{3+4 \delta} \geq \frac{1}{3}-\frac{1}{3} \delta
$$

where the first inequality follows from (2.9) and (2.11), and the second inequality from $S_{j} \leq \beta_{8}$.

Consider $q_{i}^{*} \in C_{i}(4)$. It is implied by (2.9) and (2.12) that

$$
\frac{1}{3}(1-\delta) \leq S_{i} \leq \frac{1}{3}\left(2-\frac{1}{3} \delta\right) .
$$

From (2.8) and (2.12) it follows that $S_{j}>\frac{5+5 \delta-2 \delta S_{i}}{6+4 \delta}$. In conclusion, $\left(q_{j}^{*}, q_{i}^{*}\right)$ is a Nash equilibrium with $q_{j}^{*} \in A_{j}(2)$ if and only if $q_{j}^{*}=\frac{2+3 \delta-2 \delta S_{i}}{6+4 \delta}, q_{i}^{*}=$ $\frac{1-\delta+2 \delta S_{i}}{3+2 \delta}, \frac{1}{3}(1-\delta) \leq S_{i} \leq \frac{1}{3}\left(2-\frac{1}{3} \delta\right)$, and $S_{j}>\frac{5+5 \delta-2 \delta S_{i}}{6+4 \delta}$.

\footnotetext{
${ }^{7}$ Note that, by Proposition B.1, $q_{i}^{*} \notin C_{i}(1)$. By Proposition B.1, if $q_{i}^{*} \in C_{i}(1)$, then $q_{j}^{*} \in$ $C_{j}(1,2,3,4)$
} 
$\mathbf{q}_{\mathbf{j}}^{*} \in \mathbf{A}_{\mathbf{j}}(\mathbf{3})$

It holds that

$$
\begin{aligned}
& q_{j}^{*}<S_{j}-\frac{1}{2}, \\
& \frac{5}{3}-\frac{1}{6} \delta-2 S_{i}<q_{j}^{*} \leq \frac{5}{3}-2 S_{i}, \\
& q_{i}^{*}=\sigma_{i}^{\mathrm{R}}\left(q_{j}^{*}\right)=S_{i}-\frac{1}{3} .
\end{aligned}
$$

By Proposition B.1, $q_{i}^{*} \in C_{i}(2,3,4)$. This gives the following possibilities:

$$
\begin{aligned}
& q_{i}^{*} \in C_{i}(2): q_{j}^{*}=0, \\
& q_{i}^{*} \in C_{i}(3): q_{j}^{*}=\frac{\frac{4}{3}-\frac{2}{3} \delta+2 \delta S_{j}-S_{i}}{2+2 \delta}, \\
& q_{i}^{*} \in C_{i}(4): q_{j}^{*}=\frac{2}{3}-\frac{1}{2} S_{i} .
\end{aligned}
$$

Consider $q_{i}^{*} \in C_{i}(2)$. Since $q_{j}^{*}=0$, the second inequality in (2.15) implies $S_{i} \leq \frac{5}{6}$. We have that

$$
\frac{1}{2}<S_{j}<\frac{S_{i}-\frac{4}{3}+\frac{2}{3} \delta}{2 \delta}
$$

where the first inequality follows from (2.14) and the second from $q_{i}^{*}>\beta_{5}$. By rewriting the expression (2.16), we find that $S_{i}>\frac{4}{3}+\frac{1}{3} \delta$, contradicting $S_{i} \leq 5 / 6$.

Consider $q_{i}^{*} \in C_{i}(3)$. By (2.14), it should hold that

$$
S_{j}>1 \frac{1}{6}+\frac{1}{6} \delta-\frac{1}{2} S_{i},
$$

which contradicts with $S_{j} \leq \beta_{8}$.

Consider $q_{i}^{*} \in C_{i}(4)$. It holds that

$$
\frac{2}{3}-\frac{1}{9} \delta<S_{i} \leq \frac{2}{3}
$$

where both inequalities follow from (2.15). From (2.14), it follows that

$$
S_{j}>\frac{7}{6}-\frac{1}{2} S_{i}
$$

The other constraints are redundant. In conclusion, $q_{j}^{*} \in A_{j}(3)$ if and only if $q_{i}^{*} \in C_{i}(4), S_{j}>\frac{7}{6}-\frac{1}{2} S_{i}$ and $\frac{2}{3}-\frac{1}{9} \delta<S_{i} \leq \frac{2}{3}$. 
$\mathbf{q}_{\mathbf{j}}^{*} \in \mathbf{A}_{\mathbf{j}}(\mathbf{4})$

It holds that

$$
\begin{aligned}
& q_{j}^{*}<S_{j}-\frac{1}{2}, \\
& q_{j}^{*}>\frac{5}{3}-2 S_{i} .
\end{aligned}
$$

We have

$$
q_{i}^{*}=\sigma_{i}^{\mathrm{R}}\left(q_{j}^{*}\right)=\frac{1}{2}-\frac{1}{2} q_{j} \leq \frac{1}{2} .
$$

By Proposition B. 1 and Proposition B.2, $q_{i}^{*} \in A_{i}(4) \cup B_{i}(7){ }^{8}$ This gives the following possibilities:

$$
\begin{aligned}
& q_{i}^{*} \in A_{i}(4): q_{i}^{*}=q_{j}^{*}=\frac{1}{3}, \\
& q_{i}^{*} \in B_{i}(7): q_{i}^{*}=q_{j}^{*}=\frac{1}{3},
\end{aligned}
$$

Consider $q_{i}^{*} \in A_{i}(4) \cup B_{i}(7)$. It follows from (2.17) that

$$
S_{j}>\frac{5}{6}
$$

For $q_{i}^{*} \in A_{i}(4)$, it follows from $q_{i}^{*}<S_{i}-\frac{1}{2}$ that $S_{i}>\frac{5}{6}$. Next, if $q_{i}^{*} \in B_{i}(7)$, it follows from

$$
S_{i}-\frac{1}{2} \leq q_{i}^{*}<S_{j}-\frac{1}{3}
$$

that

$$
\frac{2}{3}<S_{i} \leq \frac{5}{6} .
$$

The other constraints are redundant. In conclusion, $q_{j}^{*} \in A_{j}(4)$ if and only if $q_{i}^{*} \in A_{i}(4) \cup B_{i}(7)$ and $S_{j}>\frac{5}{6}, S_{i}>\frac{2}{3}$.

$\mathbf{q}_{\mathbf{j}}^{*} \in \mathbf{B}_{\mathbf{j}}(\mathbf{1})$

It holds that

$$
\begin{aligned}
S_{j}-\frac{1}{2} & \leq q_{j}^{*}<S_{j}-\frac{1}{3}, \\
q_{j}^{*} & <\frac{1-\delta+\delta S_{j}-2 S_{i}}{1+\delta} .
\end{aligned}
$$

${ }^{8}$ Note that Proposition B.1 excludes that $q_{i}^{*} \in A_{i}(1,2,3) \cup B_{i}(1,2,3,4,5,6)$ and $q_{j}^{*} \in A_{j}(4)$. 
We have

$$
q_{i}^{*}=\sigma_{i}^{\mathrm{R}}\left(q_{j}^{*}\right)=S_{i} \leq \frac{1}{2} .
$$

By Proposition B.1, $q_{i}^{*} \in C_{i}(2,3,4)$. This gives the following possibilities:

$$
\begin{array}{lll}
q_{i}^{*} & \in & C_{i}(2): q_{j}^{*}=0, \\
q_{i}^{*} & \in \quad C_{i}(3): q_{j}^{*}=\frac{1-S_{i}+2 \delta S_{j}-\delta}{2+2 \delta}, \\
q_{i}^{*} & \in \quad C_{i}(4): q_{j}^{*}=\frac{1}{2}-\frac{1}{2} S_{i} .
\end{array}
$$

For $q_{i}^{*} \in C_{i}(2)$, it can be found that

$$
S_{j}<\frac{1}{2 \delta}\left(S_{i}-1+\delta\right)<\frac{1}{3 \delta}(-1+\delta) \leq 0,
$$

where the first inequality follows from $q_{i}^{*}>\beta_{5}$, the second one from (2.19) and the last one from $\delta \leq 1$.

For $q_{i}^{*} \in C_{i}(3),(2.18)$ implies $\frac{5}{6}-\frac{1}{6} \delta-\frac{1}{2} S_{i}<S_{j} \leq 1-\frac{1}{2} S_{i}$. By (2.19), $S_{i}<$ $\frac{1}{3}-\frac{1}{3} \delta$. The other constraints are redundant.

Next, $q_{i}^{*} \in C_{i}(4)$ implies $S_{j}>1-\frac{1}{2} S_{i}$, whereas (2.18) implies $S_{j} \leq 1-\frac{1}{2} S_{i}$, a contradiction.

In conclusion, $q_{j}^{*} \in B_{j}(1)$ if and only if $q_{i}^{*} \in C_{i}(3)$ and $\frac{5}{6}-\frac{1}{6} \delta-\frac{1}{2} S_{i}<S_{j} \leq$ $1-\frac{1}{2} S_{i}, S_{i}<\frac{1}{3}-\frac{1}{3} \delta$.

$$
\mathbf{q}_{\mathbf{j}}^{*} \in \mathbf{B}_{\mathbf{j}}(\mathbf{2})
$$

It holds that

$$
\begin{aligned}
S_{j}-\frac{1}{2} & \leq q_{j}^{*}<S_{j}-\frac{1}{3}, \\
q_{j}^{*} & >\frac{1-\delta+\delta S_{j}+2 \delta S_{i}}{1+\delta} .
\end{aligned}
$$

We have

$$
q_{i}^{*}=\sigma_{i}^{\mathrm{R}}\left(q_{j}^{*}\right)=0 .
$$

By Proposition B.1, $q_{i}^{*} \in C_{i}(2,3,4)$. This gives the following possibilities:

$$
\begin{array}{lll}
q_{i}^{*} \in & C_{i}(2): q_{j}^{*}=0, \\
q_{i}^{*} \in & C_{i}(3): q_{j}^{*}=\frac{1+2 \delta S_{j}-\delta+\delta S_{i}}{2+2 \delta}, \\
q_{i}^{*} \in & C_{i}(4): q_{j}^{*}=\frac{1}{2} .
\end{array}
$$


For $q_{i}^{*} \in C_{i}(2)$, from $q_{i}^{*}>\beta_{5}$ it follows that $S_{j}<\frac{1}{2 \delta}\left(-1+\delta-\delta S_{i}\right) \leq 0$.

Consider $q_{i}^{*} \in C_{i}$ (3). Inequality (2.20) implies $S_{i}<\frac{1}{3 \delta}(-1+\delta) \leq 0$.

If $q_{i}^{*} \in C_{i}(4)$, it holds again that

$$
S_{i}<\frac{1}{4 \delta}\left(-1+3 \delta-2 \delta S_{j}\right)<\frac{1}{3 \delta}(-1+\delta) \leq 0,
$$

where the first inequality follows from (2.21) and the second one from $S_{j}>$ $\beta_{8}$.

In conclusion, $q_{j}^{*} \notin B_{j}(2)$.

$\mathbf{q}_{\mathbf{j}}^{*} \in \mathbf{B}_{\mathbf{j}}(\mathbf{3})$

It holds that

$$
\begin{aligned}
S_{j}-\frac{1}{2} & \leq q_{j}^{*}<S_{j}-\frac{1}{3} \\
q_{j} & \geq \max \left\{\frac{1-\delta+\delta S_{j}-2 S_{i}}{1+\delta}, \frac{2 S_{i}-2+2 S_{j}+\delta S_{j}-\left(1-2 S_{j}\right) \sqrt{(1+\delta)\left(1+\frac{1}{2} \delta\right)}}{1+\delta+2 \sqrt{(1+\delta)\left(1+\frac{1}{2} \delta\right)}}(2,23)\right. \\
q_{j} & \leq \frac{1-\delta+\delta S_{j}+2 \delta S_{i}}{1+\delta}
\end{aligned}
$$

We have

$$
q_{i}^{*}=\sigma_{i}^{\mathrm{R}}\left(q_{j}^{*}\right)=\frac{1-q_{j}+2 \delta S_{i}-\delta+\delta S_{j}-\delta q_{j}}{2+2 \delta} .
$$

By Proposition B.1, $q_{i}^{*} \in C_{i}(2,3,4)$. This gives the following possibilities:

$$
\begin{aligned}
q_{i}^{*} \in C_{i}(2): q_{i}^{*} & =\frac{1+2 \delta S_{i}-\delta+\delta S_{j}}{2+2 \delta}, q_{j}^{*}=0, \\
q_{i}^{*} \in C_{i}(3): q_{i}^{*} & =\frac{1-\delta+3 \delta S_{i}}{3+3 \delta}, q_{j}^{*}=\frac{1-\delta+3 \delta S_{j}}{3+3 \delta}, \\
q_{i}^{*} \in C_{i}(4): q_{i}^{*} & =\frac{1-3 \delta+4 \delta S_{i}+2 \delta S_{j}}{3+3 \delta}, \\
q_{j}^{*} & =\frac{1+3 \delta-2 \delta S_{i}-\delta S_{j}}{3+3 \delta} .
\end{aligned}
$$

For $q_{i}^{*} \in C_{i}(2)$, it follows from $q_{i}^{*}>\beta_{5}$ that $S_{j}<\frac{1}{3 \delta}(-1+\delta) \leq 0$.

If $q_{i}^{*} \in C_{i}(3)$, it is implied by $(2.23)$ that $\frac{1}{3}(1-\delta) \leq S_{i} \leq \frac{7}{6}-S_{j}-\frac{1}{6} \delta+$ $\frac{\sqrt{(1+\delta)\left(1+\frac{1}{2} \delta\right)}}{1+\delta}\left(\frac{5}{6}+\frac{1}{6} \delta-S_{j}\right)$. It follows from (2.22) that $S_{j}>\frac{2}{3}$. The remaining constraints are redundant. 
Consider $q_{i}^{*} \in C_{i}(4)$. By $S_{j}>\beta_{8}$ and (2.23) it follows that $2-2 S_{j}<S_{i} \leq$ $\frac{7+10 \delta+3 \delta^{2}-6 S_{j}-10 \delta S_{j}-4 \delta^{2} S_{j}+\left(5+9 \delta-6 S_{j}-8 \delta S_{j}\right) \sqrt{(1+\delta)\left(1+\frac{1}{2} \delta\right)}}{6+8 \delta+2 \delta^{2}+4 \delta \sqrt{(1+\delta)\left(1+\frac{1}{2} \delta\right)}}$. Such an $S_{i}$ only exists if $S_{j}>\frac{5+\delta}{6}$. From (2.22) it follows that $S_{i} \leq \frac{5+9 \delta-6 S_{j}-8 \delta S_{j}}{4 \delta}$. Now, there only exists an $S_{i}$ such that $2-2 S_{j}<S_{i} \leq \frac{5+9 \delta-6 S_{j}-8 \delta S_{j}}{4 \delta}$, if $S_{j}<\frac{5+\delta}{6}$, a contradiction.

In conclusion, $q_{j}^{*} \in B_{j}(3)$ if and only if $q_{i}^{*} \in C_{i}(3)$ and $\frac{1}{3}(1-\delta) \leq S_{i} \leq$ $\frac{7}{6}-S_{j}-\frac{1}{6} \delta+\frac{\sqrt{(1+\delta)\left(1+\frac{1}{2} \delta\right)}}{1+\delta}\left(\frac{5}{6}+\frac{1}{6} \delta-S_{j}\right), S_{j}>\frac{2}{3}$.

$\mathbf{q}_{\mathbf{j}}^{*} \in \mathbf{B}_{\mathbf{j}}(\mathbf{4})$

It holds that

$$
\begin{aligned}
S_{j}-\frac{1}{2} & \leq q_{j}^{*}<S_{j}-\frac{1}{3} \\
q_{j} & <\frac{2 S_{i}-2+2 S_{j}+\delta S_{j}-\left(1-2 S_{j}\right) \sqrt{(1+\delta)\left(1+\frac{1}{2} \delta\right)}}{1+\delta+2 \sqrt{(1+\delta)\left(1+\frac{1}{2} \delta\right)}} \\
q_{j} & \leq \frac{5}{3}-\frac{1}{6} \delta-2 S_{i} .
\end{aligned}
$$

We have

$$
q_{i}^{*}=\sigma_{i}^{\mathrm{R}}\left(q_{j}^{*}\right)=\frac{1-q_{j}-\frac{1}{2} \delta+\delta S_{i}}{2+\delta} .
$$

By Proposition B.1, $q_{i}^{*} \in C_{i}(2,3,4)$. This gives the following possibilities:

$$
\begin{aligned}
q_{i}^{*} \in C_{i}(2): q_{i}^{*} & =\frac{1-\frac{1}{2} \delta+\delta S_{i}}{2+\delta}, q_{j}^{*}=0, \\
q_{i}^{*} \in C_{i}(3): q_{i}^{*} & =\frac{1+2 \delta-\delta^{2}+\delta S_{i}+2 \delta^{2} S_{i}-2 \delta S_{j}}{3+5 \delta+2 \delta^{2}}, \\
q_{j}^{*} & =\frac{2-3 \delta-\delta^{2}+8 \delta S_{j}+4 \delta^{2} S_{j}+2 \delta S_{i}}{6+10 \delta+4 \delta^{2}}, \\
q_{i}^{*} \in C_{i}(4): q_{i}^{*} & =\frac{1-\delta+2 \delta S_{i}}{3+2 \delta}, q_{j}^{*}=\frac{2+3 \delta-2 \delta S_{i}}{6+4 \delta} .
\end{aligned}
$$

For $q_{i}^{*} \in C_{i}(2), q_{i}>\beta_{5}$ and inequality (2.25) imply respectively that $\frac{1}{3}<$ $S_{j}<\frac{-1+\frac{3}{2} \delta+\frac{1}{2} \delta^{2}-\delta S_{i}}{2 \delta(2+\delta)}$. However, no such $S_{j}$ exists, since this would imply that $S_{i}<\frac{1}{\delta}\left(-1+\frac{1}{6} \delta-\frac{1}{6} \delta^{2}\right) \leq 0$. 
Consider $q_{i}^{*} \in C_{i}(3)$. It follows from (2.26) and $q_{i}<\beta_{5}$ that

$$
\frac{7+9 \frac{1}{2} \delta+2 \delta^{2}-\frac{1}{2} \delta^{3}-6 S_{j}-9 \delta S_{j}-3 \delta^{2} S_{j}+\left(5+2 \delta+\delta^{2}-6 S_{j}-2 \delta S_{j}\right) \sqrt{(1+\delta)\left(1+\frac{1}{2} \delta\right)}}{6+9 \delta+3 \delta^{2}-2 \delta \sqrt{(1+\delta)\left(1+\frac{1}{2} \delta\right)}}
$$

$<S_{i} \leq 2-2 S_{j}$

and from (2.25) and $q_{i}<\beta_{5}$ that

$$
\frac{6 S_{j}+2 \delta S_{j}-5-2 \delta-\delta^{2}}{2 \delta} \leq S_{i} \leq 2-2 S_{j}
$$

There exists an $S_{i}$ such that (2.28) if and only if $S_{j}>\frac{5+\delta}{6}$ and there exists an $S_{i}$ such that (2.29) holds if and only if $S_{j} \leq \frac{5+\delta}{6}$, a contradiction.

If $q_{i}^{*} \in C_{i}(4)$, inequality (2.27) implies $S_{i} \leq \frac{1}{3}\left(2-\frac{1}{3} \delta\right)$. It follows from (2.26) and (2.25) respectively that $S_{j}>\frac{7+6 \frac{1}{2} \delta+\frac{3}{2} \delta^{2}-6 S_{i}-5 \delta S_{i}-\delta^{2} S_{i}+\left(5+5 \delta-2 \delta S_{i}\right) \sqrt{(1+\delta)\left(1+\frac{1}{2} \delta\right)}}{6+7 \delta+2 \delta^{2}+(6+4 \delta) \sqrt{(1+\delta)\left(1+\frac{1}{2} \delta\right)}}$ and $S_{j} \leq \frac{5+5 \delta-2 \delta S_{i}}{6+4 \delta}$. The other constraints are redundant.

In conclusion, $q_{j}^{*} \in B_{j}(4)$ if and only if $q_{i}^{*} \in C_{i}(4)$ and $S_{i} \leq \frac{1}{3}\left(2-\frac{1}{3} \delta\right)$, $\frac{7+6 \frac{1}{2} \delta+\frac{3}{2} \delta^{2}-6 S_{i}-5 \delta S_{i}-\delta^{2} S_{i}+\left(5+5 \delta-2 \delta S_{i}\right) \sqrt{(1+\delta)\left(1+\frac{1}{2} \delta\right)}}{6+7 \delta+2 \delta^{2}+(6+4 \delta) \sqrt{(1+\delta)\left(1+\frac{1}{2} \delta\right)}}<S_{j} \leq \frac{5+5 \delta-2 \delta S_{i}}{6+4 \delta)}$.

\section{$\mathbf{q}_{\mathbf{j}}^{*} \in \mathbf{B}_{\mathbf{j}}(\mathbf{5})$}

It holds that

$$
\begin{aligned}
S_{j}-\frac{1}{2} & \leq q_{j}^{*}<S_{j}-\frac{1}{3} \\
\frac{5}{3}-\frac{1}{6} \delta-2 S_{i} & <q_{j}<\frac{2 S_{i}-2+2 S_{j}+\delta S_{j}-\left(1-2 S_{j}\right) \sqrt{(1+\delta)\left(1+\frac{1}{2} \delta\right)}}{1+\delta+2 \sqrt{(1+\delta)\left(1+\frac{1}{2} \delta\right)}} \\
q_{j} & \geq \frac{2 S_{i}-3-\delta+4 S_{j}+3 \delta S_{j}}{3+3 \delta}
\end{aligned}
$$

We have

$$
q_{i}^{*}=\sigma_{i}^{\mathrm{R}}\left(q_{j}^{*}\right)=\frac{1-q_{j}+2 \delta S_{i}-\delta+\delta S_{j}-\delta q_{j}}{2+2 \delta} .
$$

60 
By Proposition B. $1, q_{i}^{*} \in C_{i}(2,3,4)$. This gives the following possibilities:

$$
\begin{aligned}
q_{i}^{*} \in C_{i}(2): q_{i}^{*} & =\frac{1+2 \delta S_{i}-\delta+\delta S_{j}}{2+2 \delta}, q_{j}^{*}=0, \\
q_{i}^{*} \in C_{i}(3): q_{i}^{*} & =\frac{1-\delta+3 \delta S_{i}}{3+3 \delta}, q_{j}^{*}=\frac{1-\delta+3 \delta S_{j}}{3+3 \delta}, \\
q_{i}^{*} \in C_{i}(4): q_{i}^{*} & =\frac{1-3 \delta+4 \delta S_{i}+2 \delta S_{j}}{3+3 \delta}, \\
q_{j}^{*} & =\frac{1+3 \delta-2 \delta S_{i}-\delta S_{j}}{3+3 \delta} .
\end{aligned}
$$

For $q_{i}^{*} \in C_{i}(2)$, it follows from $q_{i}>\beta_{6}$ that $S_{j}<\frac{1}{3 \delta}(-1+\delta) \leq 0$.

For $q_{i}^{*} \in C_{i}(3)$, it holds that $S_{j} \leq 1-\frac{1}{2} S_{i}$. It is implied by (2.31) that $S_{i}>\max \left\{\frac{4+5 \frac{1}{2} \delta-\frac{1}{2} \delta^{2}-3 \delta S_{j}}{6+6 \delta}, \frac{7}{6}-\frac{1}{6} \delta-S_{j}+\frac{\sqrt{(1+\delta)\left(1+\frac{1}{2} \delta\right)}}{1+\delta}\left(\frac{5}{6}+\frac{1}{6} \delta-S_{j}\right)\right\}$. From (2.30) it follows that $S_{j}>\frac{2}{3}$. The other constraints are redundant.

If $q_{i}^{*} \in C_{i}(4)$, it holds that $S_{j}>1-\frac{1}{2} S_{i}$. From (2.32) it follows that $S_{j} \leq$ $1-\frac{1}{2} S_{i}$, a contradiction.

In conclusion, $q_{j}^{*} \in B_{j}(5)$ if and only if $q_{i}^{*} \in C_{i}(3)$ and $S_{i}>\max \left\{\frac{4+5 \frac{1}{2} \delta-\frac{1}{2} \delta^{2}-3 \delta S_{j}}{6+6 \delta}, \frac{7}{6}-\right.$ $\left.\frac{1}{6} \delta-S_{j}+\frac{\sqrt{(1+\delta)\left(1+\frac{1}{2} \delta\right)}}{1+\delta}\left(\frac{5}{6}+\frac{1}{6} \delta-S_{j}\right)\right\}, \frac{2}{3}<S_{j} \leq 1-\frac{1}{2} S_{i}$.

$$
\mathbf{q}_{\mathbf{j}}^{*} \in \mathbf{B}_{\mathbf{j}}(\mathbf{6})
$$

It holds that

$$
\begin{aligned}
S_{j}-\frac{1}{2} & \leq q_{j}^{*}<S_{j}-\frac{1}{3}, \\
\frac{5}{3}-\frac{1}{6} \delta-2 S_{i} & <q_{j} \leq \frac{5}{3}-2 S_{i}, \\
q_{j} & <\frac{2 S_{i}-3-\delta+4 S_{j}+3 \delta S_{j}}{3+3 \delta} .
\end{aligned}
$$

We have

$$
q_{i}^{*}=\sigma_{i}^{\mathrm{R}}\left(q_{j}^{*}\right)=S_{i}-\frac{1}{3} .
$$


By Proposition B.1, $q_{i}^{*} \in C_{i}(2,3,4)$. This gives the following possibilities:

$$
\begin{aligned}
& q_{i}^{*} \in C_{i}(2): q_{j}^{*}=0, \\
& q_{i}^{*} \in C_{i}(3): q_{j}^{*}=\frac{\frac{4}{3}-\frac{2}{3} \delta+2 \delta S_{j}-S_{i}}{2+2 \delta}, \\
& q_{i}^{*} \in C_{i}(4): q_{j}^{*}=\frac{2}{3}-\frac{1}{2} S_{i} .
\end{aligned}
$$

For $q_{i}^{*} \in C_{i}(2)$. It holds that

$$
S_{j} \leq \frac{1}{2 \delta}\left(S_{i}-\frac{4}{3}+\frac{2}{3} \delta\right) \leq \frac{1}{2 \delta}\left(-\frac{1}{2}+\frac{2}{3} \delta\right) \leq \frac{1}{3},
$$

where the first inequality follows from (2.36) and the second one from (2.34). This contradicts with (2.33).

Consider $q_{i}^{*} \in C_{i}$ (3).It holds that $S_{j} \leq 1-\frac{1}{2} S_{i}$. From (2.33) it follows that $S_{j}>1-\frac{1}{2} S_{i}$, a contradiction.

Next, if $q_{i}^{*} \in C_{i}(4)$, it follows from (2.33) that $S_{j} \leq \frac{7}{6}-\frac{1}{2} S_{i}$. By (2.34) and by (2.35) it is implied respectively that $\frac{1}{3}\left(2-\frac{1}{3} \delta\right)<S_{i} \leq \frac{2}{3}$ and $S_{j}>$ $\frac{10+6 \delta-7 S_{i}-3 \delta S_{i}}{8+6 \delta}$. The other constraints are redundant.

In conclusion, $q_{j}^{*} \in B_{j}(6)$ if and only if $q_{i}^{*} \in C_{i}(4)$ and $\frac{1}{3}\left(2-\frac{1}{3} \delta\right)<S_{i} \leq$ $\frac{2}{3}, \frac{10+6 \delta-7 S_{i}-3 \delta S_{i}}{8+6 \delta}<S_{j} \leq \frac{7}{6}-\frac{1}{2} S_{i}$.

$$
\mathbf{q}_{\mathbf{j}}^{*} \in \mathbf{B}_{\mathbf{j}}(\mathbf{7})
$$

It holds that

$$
\begin{aligned}
S_{j}-\frac{1}{2} & \leq q_{j}^{*}<S_{j}-\frac{1}{3}, \\
q_{j}^{*} & >\frac{5}{3}-2 S_{i} .
\end{aligned}
$$

We have

$$
q_{i}^{*}=\sigma_{i}^{\mathrm{R}}\left(q_{j}^{*}\right)=\frac{1}{2}-\frac{1}{2} q_{j} \leq \frac{1}{2} .
$$

By Proposition B.1 and Proposition B.2, $q_{i}^{*} \in B_{i}(7)$. This gives the following possibilities:

$$
q_{i}^{*} \in B_{i}(7): q_{i}^{*}=q_{j}^{*}=\frac{1}{3} .
$$

For $q_{i}^{*} \in B_{j}(7)$, it follows from $S_{i}-\frac{1}{2} \leq q_{i}^{*}<S_{j}-\frac{1}{2}$ that $\frac{2}{3}<S_{i} \leq \frac{5}{6}$. From (2.37) it follows that $\frac{2}{3}<S_{j} \leq \frac{5}{6}$. The rest of the constraints is redundant.

Therefore, $q_{j}^{*} \in B_{j}(7)$ if $q_{i}^{*} \in B_{i}(7)$ and $\frac{2}{3}<S_{i} \leq \frac{5}{6}, \frac{2}{3}<S_{j} \leq \frac{5}{6}$. 
$\mathbf{q}_{\mathbf{j}}^{*} \in \mathbf{C}_{\mathbf{j}}(\mathbf{1})$

It holds that

$$
\begin{aligned}
& q_{j}^{*} \geq S_{j}-\frac{1}{3} \\
& q_{j}^{*}<\frac{1-\delta+\delta S_{j}-2 S_{i}}{1+\delta} .
\end{aligned}
$$

We have

$$
q_{i}^{*}=\sigma_{i}^{\mathrm{R}}\left(q_{j}^{*}\right)=S_{i} \leq \frac{1}{2} .
$$

By Proposition B.1 and Proposition B.2, $q_{i}^{*} \in C_{i}(1,2,3)$. This gives the following possibilities:

$$
\begin{array}{lll}
q_{i}^{*} & \in & C_{i}(1): q_{j}^{*}=S_{j}, \\
q_{i}^{*} \in & C_{i}(2): q_{j}^{*}=0, \\
q_{i}^{*} & \in & C_{i}(3): q_{j}^{*}=\frac{1-S_{i}+2 \delta S_{j}-\delta}{2+2 \delta} .
\end{array}
$$

If $q_{i}^{*} \in C_{i}(1)$, then, by $q_{i}<\beta_{4}$, it holds that $S_{j}<\frac{1}{2}-\frac{1}{2} \delta-\frac{1}{2} S_{i}$. From (2.39), it follows that $S_{i}<\frac{1}{2}-\frac{1}{2} \delta-\frac{1}{2} S_{j}$.

Consider $q_{i}^{*} \in C_{i}(2)$. It holds that

$$
S_{j}<\frac{1}{2 \delta}\left(S_{i}-1+\delta\right)<\frac{1}{3 \delta}(-1+\delta) \leq 0,
$$

where the first inequality follows from $q_{i}>\beta_{5}$ and the second one from (2.39).

For $q_{i}^{*} \in C_{i}(3)$, it follows from $q_{i}^{*} \geq \beta_{4}$ that $S_{j} \geq \frac{1}{2}-\frac{1}{2} \delta-\frac{1}{2} S_{i}$. By (2.39), it is implied that $S_{i}<\frac{1}{3}-\frac{1}{3} \delta$. From (2.38), it follows that $S_{j} \leq \frac{5}{6}-\frac{1}{6} \delta-\frac{1}{2} S_{i}$. The rest of the constraints is redundant.

In conclusion, $q_{j}^{*} \in C_{j}(1)$ if and only if $q_{i}^{*} \in C_{i}(1)$ and $S_{i}<\frac{1}{2}-\frac{1}{2} \delta-$ $\frac{1}{2} S_{j}, S_{j}<\frac{1}{2}-\frac{1}{2} \delta-\frac{1}{2} S_{i}$ or $q_{i}^{*} \in C_{i}(3)$ and $S_{i}<\frac{1}{3}-\frac{1}{3} \delta, \frac{1}{2}-\frac{1}{2} \delta-\frac{1}{2} S_{i} \leq S_{j} \leq$ $\frac{5}{6}-\frac{1}{6} \delta-\frac{1}{2} S_{i}$.

$$
\mathbf{q}_{\mathbf{j}}^{*} \in \mathbf{C}_{\mathbf{j}}(\mathbf{2})
$$

It holds that

$$
\begin{aligned}
q_{j}^{*} & \geq S_{j}-\frac{1}{3} \\
q_{j}^{*} & >\frac{1-\delta+\delta S_{j}+2 \delta S_{i}}{1+\delta} .
\end{aligned}
$$


We have

$$
q_{i}^{*}=\sigma_{i}^{\mathrm{R}}\left(q_{j}^{*}\right)=0 .
$$

By Proposition B.2, $q_{i}^{*} \in C_{i}(2,3)$. This gives the following possibilities:

$$
\begin{array}{lll}
q_{i}^{*} & \in \quad C_{i}(2): q_{j}^{*}=0, \\
q_{i}^{*} \in & C_{i}(3): q_{j}^{*}=\frac{1+2 \delta S_{j}-\delta+\delta S_{i}}{2+2 \delta} .
\end{array}
$$

For $q_{i}^{*} \in C_{i}(2)$, it follows from (2.40) that $S_{i}<\frac{1}{2 \delta}\left(-1+\delta-\delta S_{j}\right) \leq 0$.

For $q_{i}^{*} \in C_{i}(3)$, it is implied by (2.40) that $S_{i}<\frac{1}{3 \delta}(-1+\delta) \leq 0$.

In conclusion, $q_{j}^{*} \notin C_{j}(2)$ if $q_{i}^{*} \in C_{i}(2,3)$.

$$
\mathbf{q}_{\mathbf{j}}^{*} \in \mathbf{C}_{\mathbf{j}}(\mathbf{3})
$$

It holds that

$$
\begin{aligned}
q_{j}^{*} & \geq S_{j}-\frac{1}{3} \\
\frac{1-\delta+\delta S_{j}-2 S_{i}}{1+\delta} & \leq q_{j}^{*} \leq \frac{1-\delta+\delta S_{j}+2 \delta S_{i}}{1+\delta} \\
S_{i} & \leq 1-\frac{1}{2} S_{j} .
\end{aligned}
$$

We have

$$
q_{i}^{*}=\sigma_{i}^{\mathrm{R}}\left(q_{j}^{*}\right)=\frac{1-q_{j}+2 \delta S_{i}-\delta+\delta S_{j}-\delta q_{j}}{1+\delta} .
$$

By Proposition B.2, $q_{i}^{*} \in C_{i}(3)$. This gives the following possibility:

$$
q_{i}^{*} \in C_{i}(3): q_{i}^{*}=\frac{1-\delta+3 \delta S_{i}}{3+3 \delta}, q_{j}^{*}=\frac{1-\delta+3 \delta S_{j}}{3+3 \delta} .
$$

For $q_{i}^{*} \in C_{i}(3)$, it follows from $q_{i}^{*} \geq S_{i}-\frac{1}{3}$ and (2.41) respectively that $S_{i} \leq \frac{2}{3}$ and $S_{j} \leq \frac{2}{3}$.

Since $q_{i}^{*} \in C_{i}(3)$ and by (2.41), $S_{i} \leq \frac{2}{3}$ and $S_{j} \leq \frac{2}{3}$. Next, it follows from $q_{i}^{*} \geq \beta_{4}$ that $S_{j} \geq \frac{1}{3}-\frac{1}{3} \delta$. By (2.42), $S_{i} \geq \frac{1}{3}-\frac{1}{3} \delta$. The remaining constraints are redundant. $\frac{2}{3}$.

In conclusion, $q_{j}^{*} \in C_{j}(3)$ if $q_{i}^{*} \in C_{i}(3)$ and $\frac{1}{3}(1-\delta) \leq S_{i} \leq \frac{2}{3}, \frac{1}{3}(1-\delta) \leq S_{j} \leq$ 


\section{$\mathbf{q}_{\mathbf{j}}^{*} \in \mathbf{C}_{\mathbf{j}}(\mathbf{4})$}

This case does not need to be calculated here, since, by proposition B.2, it can only be combined with the situations $A_{i}$ and $B_{i}$, and all these situations are already calculated. 



\section{Chapter 3}

\section{The Impact of Bulk-Supply on a Dealer's Sales Strategy}

\subsection{Introduction}

Consider a dealer that buys its product in bulks from a manufacturer or wholesaler. On the market where it operates, the dealer has some monopolistic power. This gives the dealer the opportunity to influence its instantaneous revenue by the quantity it offers to the market. In an attempt to maximize its profit stream, it faces the following questions: Is it best to always offer the same quantity, or is there another scheme of selling the product that will increase profits? What is the best moment to order new stock? Should it try to sell many products right now and reorder quickly, or could it better postpone its reorder moment and thereby create an "artificial scarcity" to drive up price? And how does its own level of patience influence its optimal path? In this chapter, we try to answer these and some other related questions. ${ }^{1},{ }^{2}$

Examples of situations where these questions may rise are the following. The webstore that has a fixed consumer base and sells catalog books and/or cd's, i.e. products that have been on the market for a while. A dealer or webstore which offers specialized goods that are only manufactured far away

\footnotetext{
${ }^{1}$ Other papers discussing related issued, like the dealer-wholesaler arrangements and vertical integration are, among others, Bresnahan and Reiss (1985), Waterson (1982) and Greenhut and Ohta (1979).

${ }^{2}$ This chapter is based on the paper van den Berg et al. (2011b).
} 
or sold in large bulks.

In our model, a dealer tries to maximize its profit stream over an infinite horizon, in which future profits are discounted. It sells a product that it can only buy in bulks from the manufacturer. For each bulk, it pays a fixed price. It then resells the product to the consumers, where its instantaneous revenue is a concave function of the quantity it puts on the market.

We find that there exists a unique optimal order-sales strategy. When the dealer sticks to this strategy, it reorders always after the same amount of time has elapsed. The refill of stock is done exactly the moment it runs out of its current inventory. The dealer does not supply the same quantity every instant in time. Instead, it offers the largest quantity to the market right after it has refilled its stock. Then, it keeps on continuously decreasing its quantity-output, until it hits its lowest point right before reordering. When stock is refilled again, it offers the same amount of quantity to the market, as it did the first time it ordered a bulk. This pattern keeps on repeating itself. If the level of patience of the dealer decreases, its response is not to automatically increase its current sales and thereby its current instantaneous revenue. Instead, situations exists in which it actually will respond by lowering its sales-level and postponing its reordering moment.

One could also note some similarities between the topic of this chapter and the literature on inventory. However, one of the major differences between this chapter and the inventory literature is that we do not use inventory costs in our model, as we already find these results without having to implement any costs for inventory. We conjecture that inventory costs will only magnify our findings. Readers interested in literature on inventorymodels somewhat related to our model are referred to, for instance, Elmaghraby and Kescinocak (2003), Transchel and Minner (2009), Chen and Simchi-Levi (2006) and Amihud and Mendelson (1983).

This chapter is organized as follows. In Section 3.2, we build up the model. Section 3.3 is divided into two main parts. In the first part, we determine the dealer's optimal quantity-stream if the moment of reordering is fixed. In the second part, we apply the results found in the first part to determine the best reorder moment. In Section 3.4, we analyze the comparative statics. A discussion and ideas for possible extensions are given in the last section. 


\subsection{The model}

We search for the profit-maximizing strategy of a single dealer that sells a nonperishable good. The manufacturer delivers the good in batches of size $S$ for a cost of $K>0$ per batch. The size of the batches $S$ is constant and exogenously determined by the manufacturer. The dealer's inventory-level can never become negative. That is, backlogging is not allowed. It can choose when to order new stock and how much it is willing to sell from its stock every moment in time. Newly ordered stock is delivered instantly. ${ }^{3}$ Time is continuous and infinite and revenue streams and costs are discounted with a rate of $r>0$.

The variable $q(t) \geq 0$, i.e. the quantity the dealer sells at moment $t$, is the control variable. It is assumed that $q(t)$ is a piecewise continuous function of $t$ on any finite interval of $[0, \infty)$ and does not have any removable discontinuities. A function $q(t)$ is said to be piecewise continuous on an interval if the interval can be broken into a finite number of subintervals on which the function is continuous on each open subinterval and has a finite limit at the endpoints of each subinterval. A removable discontinuity is a point $x$ at which $\lim _{t \uparrow x} q(t)=\lim _{t \downarrow x} q(t) \neq q(x)$. Define $Q$ as the set of functions that satisfy these assumptions.

The instantaneous revenue the dealer receives for selling a quantity of $q$ is given by the general revenue function $R$. The function $R$ is assumed to be strictly positive on some interval $(0, A)$, on which it is twice-continuously differentiable, has a unique maximum at $q^{m}$, such that $0<q^{m}<A$, and is strictly concave. For $q=0$ and $q \geq A, R(q)=0$.

Let $X(t)$ be the inventory-level of the dealer at time $t \in \mathbb{R}_{+}, q(t)$ is the amount of stock the dealer sells at $t \in[0, \infty)$ and $T_{1}, T_{2}, T_{3}, \ldots$ are the reordermoments. We assume that at $t=0$, the dealer starts with exactly $S$ in stock. Without loss of generality, we assume that $0=T_{0}<T_{i}<T_{i+1}$ for all $i \in \mathbb{N}$.

Under the assumptions given above, let $w^{*}$ be the maximum discounted revenue stream that the dealer can receive for selling a single batch of size $S$. We assume that $K<w^{*}$.

The dealer's objective is to maximize its discounted profit stream over an infinite horizon. It does so by choosing $q(t)$ and its reorder moments optimally, subject to some restrictions. It is not possible to sell a negative quantity. Its initial stock is equal to $S$ and the decrease in stock at moment

\footnotetext{
${ }^{3}$ This model can easily be extended to a model in which it takes $x$ time to deliver the good. This will have no major effect on the results.
} 
$t$ is equal to the amount sold $q(t)$. Stock can never become negative and stock increases with $S$ the moment the dealer reorders. This makes the optimal control problem of the dealer the following.

$$
\begin{array}{cl}
\underset{\substack{q \in Q, T_{1}, T_{2}, \ldots \in \mathbb{R}_{+}, T_{1}<T_{2}<\ldots}}{\text { subject to }} & \sum_{i=0}^{\infty}\left(\int_{T_{i}}^{T_{i+1}} e^{-r t} R(q(t)) d t-e^{-r T_{i}} K\right), \\
& q(t) \geq 0, \\
& X(0)=S, \\
& \dot{X}(t)=-q(t), \\
& X(t) \geq 0, \\
& X\left(T_{i}\right)=\lim _{t \uparrow T_{i}} X(t)+S, \text { for all } i \geq 1 .
\end{array}
$$

A feasible strategy is a tuple $\left(q, T_{1}, T_{2}, \ldots\right)$ such that $q \in Q, 0<T_{1}<T_{2}<$ $\ldots \in \mathbb{R}$, such that $X(t) \geq 0$ for all $t \geq 0$. By $\Sigma$ we denote the set of all feasible strategies.

\subsection{The control problem}

Before we start analyzing the specifics of this optimal control problem, we can reduce the set of possible optimal strategies with the help of the following lemma. This lemma shows that a dealer will never reorder before it has sold all of its inventory. And, the moment the dealer runs out of inventory, it reorders.

The amount of stock the dealer owns at $t \in[0, \infty)$ when it plays strategy $\sigma \in \Sigma$ is defined by $S(\sigma, t)$. The profit a dealer receives when it plays according to $\sigma \in \Sigma$ is denoted by $\pi(\sigma)$. In an optimal strategy, the dealer will reorder exactly the moment it runs out of stock, but never before that.

Lemma 3.3.1. In an optimal strategy $\sigma$, for every $i=0,1,2, \ldots$,

(a) $S(\sigma, t)>0$ for all $t \geq 0$,

(b) $\lim _{t \uparrow T_{i+1}} S(\sigma, t)=0$.

Proof First, we will show that the dealer will not reorder before it is out of stock. Let $\sigma=\left(q, T_{1}, T_{2}, \ldots\right) \in \Sigma$ be a strategy of the dealer, in which for some $T_{k}, k \in \mathbb{N}, \lim _{t \uparrow T_{k}} S(\sigma, t)>0$. This implies that here is an $\varepsilon \in \mathbb{R}$ such that $S\left(\sigma, T_{k}+\varepsilon\right)>S$ and $T_{k}+\varepsilon<T_{k+1}$. Let $\tilde{T}_{k}=T_{k}+\varepsilon$ and define $\tilde{\sigma}=\left(q, T_{1}, T_{2}, \ldots, T_{k-1}, \tilde{T}_{k}, T_{k+1}, \ldots\right)$. This new strategy $\tilde{\sigma}$ is feasible, since the dealer has positive stock at every $t \in \mathbb{R}$. And since the dealer in both $\sigma$ and 
$\tilde{\sigma}$ sells its stock according to the same function $q$, discounted revenue is the same for both strategies. Cost are also the same for every order, except for the $k^{\prime}$ th one. That is,

$$
\pi(\tilde{\sigma})=\pi(\sigma)+\left(e^{-r T_{k}}-e^{-r \tilde{T}_{k}}\right) K .
$$

Since, by definition, $\tilde{T}_{k}>T_{k}$, it holds that $\pi(\tilde{\sigma})>\pi(\sigma)$ and the dealer therefore strictly prefers $\tilde{\sigma}$ over $\sigma$.

Secondly, the dealer will reorder immediately the moment it runs out of stock. Let there be a period of length $x$ in which the dealer is out of stock and does not reorder. After that period, the dealer orders new stock and makes from that period onwards a discounted profit of $R$. This means that at the beginning of period $x$, the future profit of the dealer is $e^{-r x} R$. This would have been $R>e^{-r x} R$, if the dealer would not have waited for a length of $x$ to reorder.

This lemma is a direct result of the effect of the discount factor $r$. That the dealer does not reorder when it still has stock, is because it is more expensive to order now than to order later. If a dealer can postpone ordering stock without having to change its output, i.e. without decreasing its revenue, this will always increase profits. The dealer has to stop sales when it runs out of stock, since backlogging is not allowed. By assumption, we know that the revenue the dealer makes on the new stock exceeds the costs of buying it. Therefore, the longer it waits with ordering new stock, the longer it takes before it can make profit on the new batch. And because of the discounting of future profits, the less profit it will get for the new stock.

\section{Revenue maximization in between ordering moments}

Lemma 3.3.1 makes it possible to break the main problem up into parts and to first analyze the optimal sales strategy in between ordering moments. W.l.o.g. let $T$ be the moment at which the dealer reorders for the first time. At this point, we analyze the problem for a given $T$ that is a candidate for a possible maximizing strategy, but is not necessarily optimally chosen yet. We can already say the following about this $T$. Note that in a strategy that maximizes total profit, $q(t) \leq q^{m}$ for all $t \in[0, T)$. The reason for this is that selling more than $q^{m}$ instead of selling $q^{m}$ during an interval of length $x$ will decrease revenues for that interval, while at the same time the dealer runs out of stock more quickly, i.e., discounted costs for buying new stock increase. 
Therefore, it must hold that the minimum time between reordering in the optimal sales strategy is $T^{m}=S / q^{m}$ and we can restrict the set of times between consecutive ordering moments from here onwards to $T \geq T^{m}$.

The dealer faces the following problem. Note that it faces a similar optimization problem in between any two, consecutive ordering moments.

$$
\max _{q \in Q} \int_{0}^{T} e^{-r t} R(q(t)) d t
$$

subject to

$$
\begin{aligned}
\dot{X}(t) & =-q(t), \\
q(t) & \geq 0, \\
X(0) & =S, \\
X(T) & =0 .
\end{aligned}
$$

The first restriction, (3.2), expresses the change in inventory at period $t$ when the dealer sells $q(t)$ of its inventory. Equation (3.3) states that the dealer can only put a nonnegative quantity on the market every moment in time. That a dealer only reorders when it is out of stock, is expressed by (3.4) and (3.5). At the reordering moment, the dealer has $S$ in stock. As time approaches the next ordering moment, the remaining stock approaches zero. Condition (3.5) has been stated as $X(T)=0$ instead of $\lim _{t \uparrow T} X(t)=0$, since there is no reordering in the subproblem.

To find the solution to this problem, we take the Hamiltonian. The Hamiltonian of this problem is:

$$
H=e^{-r t} R(q(t))-\pi(t) q(t)
$$

and, since the control variable $q(t)$ is restricted by (3.3), the Lagrangian is

$$
L=e^{-r t} R(q(t))-\pi(t) q(t)+\lambda(t) q(t) .
$$

Given that the revenue function is concave in $q$, solving the following equa- 
tions is necessary and sufficient to find the maximum:

$$
\begin{aligned}
\frac{\partial L}{\partial q(t)} & =e^{-r t} R^{\prime}(q(t))-\pi(t)+\lambda(t)=0 \\
\frac{\partial L}{\partial \pi(t)} & =\dot{X}(t)=-q(t) \\
\frac{\partial L}{\partial X(t)} & =-\dot{\pi}(t)=0 \\
\lambda(t) q(t) & =0 \\
\lambda(t) & \geq 0 \\
q(t) & \geq 0 \\
X(0) & =S \\
X(T) & =0
\end{aligned}
$$

From (3.9) we can easily see that there is a constant $c$ such that

$$
\pi(t)=c \text { for all } t \in[0, T) .
$$

Combining this with (3.7), we find that

$$
e^{-r t} R^{\prime}(q(t))+\lambda(t)=\pi(t)=c .
$$

In the following lemma, we will show that (3.15) implies that the dealers sells its stock continuously, until it is out of it.

Lemma 3.3.2. Let $q\left(t_{1}\right)>0$ for some $t_{1} \in[0, T)$, then $q(t)>0$ for all $t \in\left[0, t_{1}\right]$.

Proof Let $q(t)=0$ for some $t \in\left[0, t_{1}\right)$. Since $q\left(t_{1}\right)>0,(3.10)$ and (3.15) imply that

$$
\begin{aligned}
c & =e^{-r t_{1}} R^{\prime}\left(q\left(t_{1}\right)\right) \\
& \leq e^{-r t_{1}} R^{\prime}(0) \\
& <e^{-r t} R^{\prime}(0) \\
& <e^{-r t} R^{\prime}(0)+\lambda(t) \\
& =c .
\end{aligned}
$$

This is a contradiction.

Lemma 3.3.2 implies that there is a unique $0<\hat{t} \leq T$ such that $q(t)>0$ for $t<\hat{t}$ and $q(t)=0$ for $t \geq \hat{t}$. Since $q(t)=0$ for $t \geq \hat{t}$, the dealer does not sell 
any of its stock from $\hat{t}$ till $T$ and as a result, $X(\hat{t})=X(T)$. By (3.5), $X(T)=0$ and thus $X(\hat{t})=0$. That is, the moment the dealer stops selling should be the moment it runs out of stock.

By (3.10) and $q(t)>0$ for all $t \in[0, \hat{t})$, it must hold that $\lambda(t)=0$ for all $t \in[0, \hat{t})$.

Lemma 3.3.3. The control variable $q(t)$ is continuous for $t \in[0, T]$.

Proof From Lemma 3.3.2, we know that there is a unique $\hat{t}$ such that $q(t)>$ 0 for all $t<\hat{t}$. For $q(t)>0$, by (3.15), $c=e^{-r t} R^{\prime}(q(t))$. Since $R^{\prime}(q)$ and $e^{-r t}$ are continuous functions, $q(t)$ must be continuous for all $t<\hat{t}$. For $t>\hat{t}, q(t)=0$ and automatically continuous. All is left to show that $q(t)$ is continuous at $t=\hat{t}$. For $t \uparrow \hat{t}, e^{-r t} R^{\prime}(q(t))=c$ and at $\hat{t}, e^{-r t} R^{\prime}(0)+\lambda(\hat{t})=c$. By (3.11), $\lambda(\hat{t}) \geq 0$.

This implies

$$
\begin{aligned}
c & =\lim _{t \uparrow \hat{t}} e^{-r t} R^{\prime}(q(t)) \\
& \leq \lim _{t \uparrow \hat{t}} e^{-r t} R^{\prime}(0) \\
& =e^{-r \hat{t}} R^{\prime}(0) \\
& \leq e^{-r \hat{t}} R^{\prime}(0)+\lambda(\hat{t}) \\
& =c .
\end{aligned}
$$

For this to hold true, no inequality can be strict and $\lambda(\hat{t})=0$. This implies that $\lim _{t \uparrow \hat{t}} e^{-r t} R^{\prime}(q(t))=e^{-r \hat{t}} R^{\prime}(0)$ and therefore, $q(t)$ must be continuous at $t=\hat{t}$.

With the help of Lemmas 3.3.2 and 3.3.3, the function $q$ that maximizes (3.1), subject to (3.2)-(3.5), satisfies the following conditions.

Proposition 3.3.4. a) Let $q$ be a solution of (3.1)-(3.5). Then there are $c \in \mathbb{R}$ and $\hat{t} \in\left[T^{m}, T\right]$ such that

$$
\begin{gathered}
R^{\prime}(q(t))=c e^{r t}, \text { for } t \leq \hat{t}, \\
\int_{0}^{\hat{t}} q(t) d t=S,
\end{gathered}
$$

and

$$
q(t)=0 \text { for } t \geq \hat{t} .
$$

b) The solution to the system in a) is unique. 
Proof a) Follows directly from (3.7)-(3.14) and Lemmas 3.3.2 and 3.3.3.

b) Let $c, \hat{t}$ and $q^{*}$ be a solution to the system in a). While keeping the $\hat{t}$ fixed, a strict increase (decrease) in $c$ will strictly decrease (increase) $q^{*}(t)$ for all $t \in[0, \hat{t}]$ and this violates (3.17). Vice versa, while keeping this $\hat{t}$ fixed, a strict increase (decrease) in $q^{*}(t)$ for some $t \in[0, \hat{t}]$ violates (3.16), unless $c$ is strictly decreased (increased). However, then $q^{*}(t)$ must be increased (decreased) for all $t \in[0, \hat{t}]$ to not violate (3.16), thereby automatically violating (3.17). That is, every $\hat{t}$ that is a solution to the system in Proposition 3.3.4, is accompanied by a uniquely determined $c$ and $q^{*}$. Left to show is that $\hat{t}$ itself is uniquely determined. If $\hat{t}<T$, then by the continuity of $q^{*}(t)$, $q(\hat{t})=0$. A strict increase (decrease) in $\hat{t}$ results in a strict decrease (increase ) of $q(\hat{t})$ and $q$ is no longer continuous at $\hat{t}$. If $\hat{t}=T, q(t)>0$ for all $t \in[0, T]$. A strict decrease ${ }^{4}$ of $\hat{t}$ results in a strict increase of $q^{*}(\hat{t})$ and $q^{*}$ is no longer continuous at $\hat{t}$. Ergo, for every combination of $r, S$ and $T \geq T^{m}$, the system gives a unique solution for $c, \hat{t}$ and $q^{*}$.

From now on, given $T$, the unique $q$ and $c$ in Proposition 3.3.4 are denoted by $q^{T}$ and $c^{T}$.

Note that, if $\hat{t}<T$, a further increase in $T$ will not change $q^{T}, c^{T}$ and $\hat{t}$ anymore. And the dealer is out of stock before it reorders. From Lemma 3.3.1 we know this will not happen if the dealer can choose its own reorder moment. This makes it possible to not only have a lower bound for $T$, but also set an upper bound on it. We already know that the moment the dealer is out of stock, $\hat{t}$, is uniquely defined by $T$. We define $\hat{T}$ to be the maximal $T \in \mathbb{R}_{+}$for which the associated $\hat{t}$ is equal to $T$. So, if the dealer were to choose the time between ordering moments, $\hat{T}$ is its upper bound. From here onwards, we assume that $T \in\left[T^{m}, \hat{T}\right]$. This makes it possible to restate Proposition 3.3.4 as:

Proposition 3.3.5. If $T \in\left[T^{m}, \hat{T}\right]$, there are unique $c^{T}$ and $q^{T}$ such that

$$
\begin{gathered}
R^{\prime}\left(q^{T}(t)\right)=c^{T} e^{r t}, \text { for } t \leq T, \\
\int_{0}^{T} q^{T}(t) d t=S
\end{gathered}
$$

Given what is now known about $q^{T}$ and $c^{T}$, we argue next that $c^{T} \geq 0$. We will also show how a change in $t$ or $T$ affects $q^{T}$ and $c^{T}$. Note that, since $c^{T} e^{r t}$ is differentiable to $t$, by equation (3.19) so is $q^{T}(t)$ for $t \leq T$.

\footnotetext{
${ }^{4}$ Note that increasing $\tilde{t}$ is not possible here.
} 
Proposition 3.3.6. a) $c^{T} \geq 0$.

b)

$$
\begin{aligned}
& \frac{\partial q^{T}(t)}{\partial t} \leq 0, \\
& \frac{\partial q^{T}(t)}{\partial T} \leq 0
\end{aligned}
$$

and

$$
\frac{\partial c^{T}}{\partial T} \geq 0
$$

Proof a) As mentioned in the beginning of this section, $q^{T}(t) \leq q^{m}$ for all $t \in[0, T]$. For $q^{T}(t) \leq q^{m}$ it holds that $R^{\prime}\left(q^{T}(t)\right) \geq 0$. Since $e^{r t}>0$ for all $t \in\left[0, T^{m}\right]$, from (3.19) it follows directly that $c^{T} \geq 0$.

b) See appendix.

That is, the derivatives learn us the following. In between two arbitrary consecutive ordering moments, the dealer sells relatively the largest part of its stock in the beginning and continuously sells less and less, until it reorders. If the dealer increases the time it will take before it has sold all of its stock, the amount it sells at every instant in time decreases and $c^{T}$ goes up. Note that, even though the dealer's quantity output continuously decreases until it reorders, this does not imply that its sales right before reordering are equal to zero in the limit.

Example 3.3.7. Let $R(q)=q(1-q)$. Then, from (3.19) it follows that $q^{T}(t)=$ $\frac{1}{2}\left(1-c^{T} e^{r t}\right)$. Substituting $q^{T}(t)$ in (3.20), we get

$$
\begin{aligned}
S & =\int_{0}^{T} \frac{1}{2}\left(1-c^{T} e^{r t}\right) d t \\
& =\frac{1}{2}\left[t-\frac{1}{r} c^{T} e^{r t}\right]_{0}^{T} \\
& =\frac{1}{2}\left(T-\frac{1}{r} c^{T} e^{r T}+\frac{1}{r} c^{T}\right) .
\end{aligned}
$$

This gives

$$
c^{T}=\frac{r(2 S-T)}{1-e^{r T}}
$$

and

$$
q^{T}(t)=\frac{1}{2}\left(1-\frac{e^{r t} r(2 S-T)}{1-e^{r T}}\right)
$$




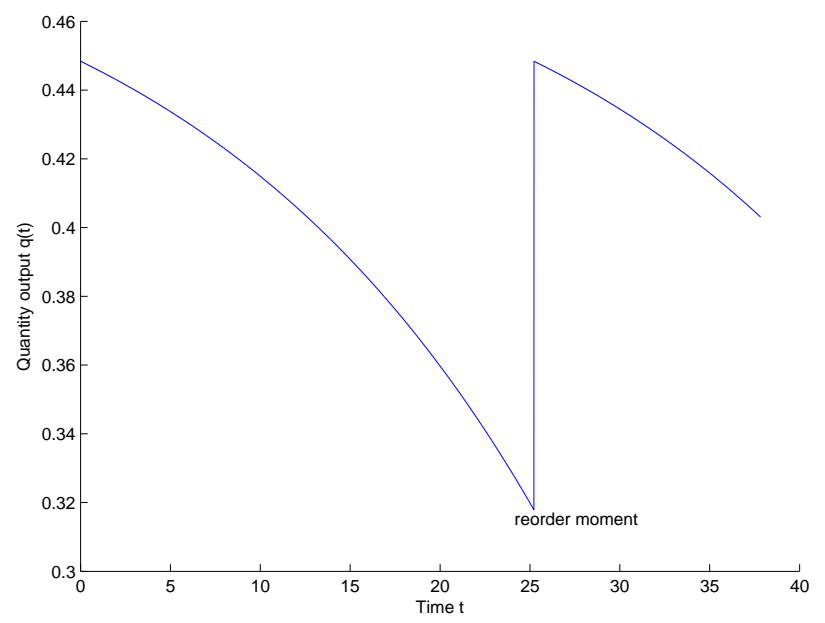

Figure 3.1: Output at any moment $t$ for a linear demand function with $S=10, r=$ 0.05 and $K=2$.

Figure 3.1 shows how output depends on time for a linear demand function. The lower and upper bound for $T$ are calculated as follows. The monopoly output is $q^{m}=\frac{1}{2}$, which gives $T^{m}=2 S$. The upper bound for $T$ should equate

$$
\begin{aligned}
q^{T}(\hat{T}) & =\frac{1}{2}\left(1-\frac{e^{r \hat{T}} r(2 S-\hat{T})}{1-e^{r \hat{T}}}\right) \\
& =0
\end{aligned}
$$

This implies that $\hat{T}$ can be found by solving for

$$
e^{-r \hat{T}}-1=r(2 S-\hat{T}) .
$$

In the model, we have assumed that $K<w^{*}$, where $w^{*}$ is the maximal discounted revenue the dealer can make on a single batch. With the help of what we have analyzed so far, we can now calculate $w^{*}$.

Lemma 3.3.8. The maximum discounted revenue a dealer can earn on a batch of size $S$ is $w^{*}=\int_{0}^{\hat{T}} e^{-r t} R\left(q^{\hat{T}}(t)\right) d t$.

Proof The maximum revenue a dealer can make is given by

$$
\max _{\bar{t}} V(\bar{t})=\max _{\bar{t} \in\left[T^{m}, \hat{T}\right]} \int_{0}^{\bar{t}} e^{-r t} R\left(q^{\bar{t}}(t)\right) d t
$$


Differentiating this, we get

$$
\begin{aligned}
\frac{d V(\bar{t})}{d \bar{t}} & =e^{-r \bar{t}} R\left(q^{\bar{t}}(\bar{t})\right)+\int_{0}^{\bar{t}} e^{-r t} R^{\prime}\left(q^{\bar{t}}(t)\right) \frac{d q^{\bar{t}}(t)}{d \bar{t}} d t \\
& =e^{-r \bar{t}} R\left(q^{\bar{t}}(\bar{t})\right)+\int_{0}^{\bar{t}} c^{\bar{t}} \frac{d q^{\bar{t}}(t)}{d \bar{t}} d t \\
& =e^{-r \bar{t}} R\left(q^{\bar{t}}(\bar{t})\right)-c^{\bar{t}} q^{\bar{t}}(\bar{t}) .
\end{aligned}
$$

These equalities follow from (3.19) and (2.22). Again using (3.19) to substitute, this gives

$$
\frac{d V(\bar{t})}{d \bar{t}}=e^{-r \bar{t}}\left(R\left(q^{\bar{t}}(\bar{t})\right)-R^{\prime}\left(q^{\bar{t}}(\bar{t})\right) q^{\bar{t}}(\bar{t})\right) .
$$

Note that $\frac{\partial}{\partial \bar{t}}\left(R\left(q^{\bar{t}}(\bar{t})\right)-R^{\prime}\left(q^{\bar{t}}(\bar{t})\right) q^{\bar{t}}(\bar{t})\right)=-\frac{\partial q}{\partial \bar{t}} R^{\prime \prime}\left(q^{\bar{t}}(\bar{t})\right) q^{\bar{t}}(\bar{t})<0$. So, the righthand-side (3.27) strictly decreases as $\bar{t}$ increases. Note that, by definition, (3.27) is 0 when $q^{\bar{t}}(\bar{t})=0$. This must be the $\bar{t}$ that maximizes (3.27), since (3.27) strictly decreases in $\bar{t}$. Therefore, the $\bar{t}$ that maximizes the revenue on a specific batch $S$ is found by solving $q^{\bar{t}}(\bar{t})=0$. This implies that $\bar{t}=\hat{T}$ and the dealer will only reorder if

$$
K \leq \int_{0}^{\hat{T}} e^{-r t} R\left(q^{\hat{T}}(t)\right) d t .
$$

This lemma implies that the assumption $K<w^{*}$ is equivalent to $K<$ $\int_{0}^{\hat{T}} e^{-r t} R\left(q^{\hat{T}}(t)\right) d t$.

\section{The best moment to order}

So far, we have analyzed the best sales strategy of the dealer when its ordering moments are fixed. In this section we will analyze how it can best choose those ordering moments. Let $T^{*}$ be the optimal time after which the dealer wants to reorder. We already know that $T^{*} \in\left[T^{m}, \hat{T}\right]$. We will now demonstrate in a few steps that, if there exists an optimal strategy, there exists an optimal strategy that is stationary. ${ }^{5}$ Let $\Sigma^{*} \subset \Sigma$ be the set of strategies that maximize profits and let $\pi^{*}$ be the maximal discounted sum of profits the dealer can make over all its future sales. First, we will show that there is an

\footnotetext{
${ }^{5}$ See definition below.
} 
optimal strategy in which the dealer always reorders after the same amount of time has elapsed.

Lemma 3.3.9. Let $\sigma=\left(q, T_{1}, T_{2}, \ldots\right) \in \Sigma^{*}$. Then there exists an optimal strategy $\sigma^{\prime}=\left(q_{1}^{\prime}, T_{1}^{\prime}, T_{2}^{\prime}, \ldots\right) \in \Sigma^{*}$ such that $T_{n}^{\prime}=n T_{1}$ for all $n \in \mathbb{N}$.

Proof Let $\sigma_{1}=\sigma\left(q^{*}, T_{1}^{*}, T_{2}^{*}, \ldots\right) \in \Sigma^{*}$ and let $\sigma_{2}=\sigma\left(q^{*}\left(t+T_{1}^{*}\right), T_{2}^{*}-T_{1}^{*}, T_{3}^{*}-\right.$ $\left.T_{1}^{*}, \ldots\right)$. Since $\sigma_{1}$ is an optimal strategy and $\sigma_{2}$ is exactly $\sigma_{1}$, but then beginning at moment $T_{1}^{*}$, it should hold as well that $\sigma_{2} \in \Sigma^{*}$. If not, playing $\sigma_{1}$ implies the dealer can strictly improve its profits from $T_{1}^{*}$ onwards by deviating from $\sigma_{1}$ and $\sigma_{1}$ would not be an optimum strategy. Let $v_{1}(\sigma)$ be the profit the dealer makes up to the first ordering moment, if it plays according to strategy $\sigma$. Since $\sigma_{2} \in \Sigma^{*}$, we know the following:

$$
\begin{aligned}
\pi^{*} & =\pi\left(\sigma_{1}\right) \\
& =v_{1}\left(\sigma_{1}\right)+e^{-r T_{1}^{*} \pi\left(\sigma_{2}\right)} \\
& =v_{1}\left(\sigma_{1}\right)+e^{-r T_{1}^{*}} \pi^{*} .
\end{aligned}
$$

Rewriting this last expression, we get the following.

$$
\begin{aligned}
\pi^{*} & =v_{1}\left(\sigma_{1}\right) * \frac{1}{1-e^{-r T_{1}^{*}}} \\
& =\left(\sum_{i=0}^{\infty} e^{-i r T_{1}^{*}}\right) * v_{1}\left(\sigma_{1}\right) \\
& =\pi\left(\sigma\left(\tilde{q}(t), T_{1}^{*}, 2 T_{1}^{*}, 3 T_{1}^{*}, \ldots\right)\right),
\end{aligned}
$$

where

$$
\tilde{q}(t)=\left\{\begin{array}{cc}
q^{*}(t) & \text { for } t<T_{1}^{*} \\
q^{*}\left(t-i T_{1}^{*}\right) & \text { for } i T_{1}^{*} \leq t<(i+1) T_{1}^{*}
\end{array} .\right.
$$

This implies that repeatedly playing $q^{*}(t)$ as it is defined between 0 and $T_{1}$, results into the dealer receiving the maximum discounted sum of profits.

We have shown so far that, if there exists an optimal strategy, there exists an optimal strategy in which the dealer always orders after the same amount of time. We can now show that there exists an optimal strategy that is stationary. A stationary strategy is a strategy in which the dealer bases its choice of action only on the state variable $X(t)$, such that, when $X\left(t_{1}\right)=X\left(t_{2}\right)$, then $q\left(t_{1}\right)=q\left(t_{2}\right)$ and $O\left(X\left(t_{1}\right)\right)=O\left(X\left(t_{2}\right)\right)$ for all $t_{1}, t_{2} \in \mathbb{R}^{+}$. The variable $O(x)$ is binary and takes on the values 1 or 0 , respectively, when the dealer reorders or does not reorder, given that the remaining stock 
is equal to $x$. It is already known from Lemma 3.3.1 that the dealer reorders exactly at the moment it runs out of stock. That means that in any optimal strategy, $O(0)=1$ and $O(x)=0$ for all $x \neq 0$. Therefore, to prove that there exists an optimal strategy that is stationary, it is sufficient to show that, when $X\left(t_{1}\right)=X\left(t_{2}\right)$, then $q\left(t_{1}\right)=q\left(t_{2}\right)$ for all $t_{1}, t_{2} \in \mathbb{R}^{+}$.

Theorem 3.3.10. There exists an optimal strategy that is stationary.

Proof If the dealer never reorders, this is true by definition. If the dealer reorders, it is an optimal strategy to keep the time in between ordering moments fixed for some optimally chosen $T^{*}$, as is shown in Lemma 3.3.9. Take two optimal, arbitrary chosen, reordering moments $T_{i}=i T^{*}$ and $T_{j}=j T^{*}$, where $i \neq j$ and $i, j \in \mathbb{N}$, from an optimally chosen strategy in which the time between ordering moments is fixed. Note that $X\left(T_{i}\right)=X\left(T_{j}\right)$. For this strategy it should hold that

$$
\int_{T_{i}}^{T_{i}+T^{*}} e^{-r\left(t-T_{i}\right)} R(q(t)) d t=\int_{T_{j}}^{T_{j}+T^{*}} e^{-r\left(t-T_{j}\right)} R(q(t)) d t .
$$

Let $\tilde{q}(t)$ maximize the revenues in between $T_{i}$ and $T_{i}+T^{*}$. Then $\tilde{q}\left(T_{i}-T_{j}+t\right)$ should also maximize revenues for $t \in\left[T_{j}, T_{j}+T^{*}\right.$ ) and (3.28) holds. Define $q(t)=\tilde{q}\left(T_{i}-T_{j}+t\right)$ for all $j \in \mathbb{N}$. This strategy maximizes revenue in between any two ordering moments, and since the ordering moments are chosen optimally, this must be a profit maximizing strategy. For this strategy it holds that whenever $X\left(t_{1}\right)=X\left(t_{2}\right)$ it must be that $q\left(t_{1}\right)=q\left(t_{2}\right)$ and therefore, this strategy is stationary.

We have shown that, if there exist an optimal strategy, there exist an optimal strategy that is stationary. The following theorem shows that there exists an optimal strategy. Furthermore, there is an explicit equation to find the best moment to order and thereby an explicit way of how to determine the optimal strategy.

Theorem 3.3.11. There exists a unique stationary strategy that maximizes total profits. In this strategy, new inventory is ordered after $T^{*}$ periods, where $T^{*}$ can be found by solving

$$
\left(1-e^{-r T^{*}}\right)\left(e^{-r T^{*}} R\left(q^{T^{*}}\left(T^{*}\right)\right)-c^{T^{*}} q^{T^{*}}\left(T^{*}\right)\right)+r e^{-r T^{*}}\left(K-\int_{0}^{T^{*}} e^{-r t} R\left(q^{T^{*}}(t)\right) d t\right)=0 .
$$


Proof See appendix.

Since $T^{*}$ is unique, we know that there is only one stationary strategy that maximizes total profit. This is also the only optimal strategy. If there is a strategy $\tilde{\sigma}$ that is not stationary, but optimal, then there must be periods where the time between ordering is not equal to $T^{*}$, but to some $\tilde{T} \neq T^{*}$. This implies that there must exist a non-stationary optimal strategy in which the first reordering moment comes after $\tilde{T}$ time. By Lemma 3.3.2, this means there must be an optimal strategy that is stationary and in which reordering is done every time after $\tilde{T}$ time. This is a contradiction. Given that $T^{*}$ is the unique optimal reordering time, $q^{T^{*}}$ is also unique by Proposition 3.3.4. Therefore, there is a unique optimal strategy; this strategy is stationary and reordering is done after $T^{*}$ time has elapsed.

We have found that there is a unique optimal strategy, and that this strategy is stationary. The dealer always reorders after $T^{*}$ time has elapsed and its sales strategy in between any two arbitrary, consecutive reorderingmoments is always the same. Note that this implies that, even though the dealer keeps its discounted instantaneous revenue constant in between ordering moments, its discounted instantaneous revenue decreases every time it starts selling a new batch.

\subsection{Comparative statics}

In this section, it is analyzed how $T^{*}$ changes, when $S, r$ or $K$ changes. The discounted profit of the dealer when it plays according to its optimal strategy and $K=50$ is shown in Figure 3.2. As $T^{*}$ changes, so do $q^{T}$ and $c^{T}$, by Proposition 3.3.5. Besides that, $q^{T}$ and $c^{T}$ are influenced directly by a change of either $S$ or $r$. That is, if $S, r$ and $K$ are no longer constants, but taken as variables, $T^{*}$ becomes a function of $S, r$ and $K$, or formally, $T^{*}(S, r, K)$. And $q^{T^{*}}(t)$ and $c^{T^{*}}$ become functions of $S, r$ and $K$ as well, given respectively by $q^{T^{*}(S, r, K)}(S, r, t)$ and $c^{T^{*}(S, r, K)}(S, r)$. However, to avoid lengthy expression in this section, we often omit the arguments, if no confusion can arise. Therefore, $q^{T^{*}(S, r, K)}(S, r, t)$ is denoted as $q(t)$ and $c^{T^{*}(S, r, K)}(S, r)$ is denoted as $c$. The partial derivatives are given by $\frac{\partial c}{\partial T}, \frac{\partial c}{\partial r}, \frac{\partial c}{\partial S}, \frac{\partial q}{\partial T}, \frac{\partial q}{\partial r}$ and $\frac{\partial q}{\partial S}$.

Proposition 3.4.1. If the dealer plays according to its optimal strategy, an increase in the costs $K$ of buying new stock will increase the time in between ordering moments. 
Proof In the appendix it is shown that

$$
\begin{aligned}
T_{K}^{* \prime} & =\frac{r e^{-r T^{*}}}{\left(1-e^{-r T^{*}}\right) q\left(T^{*}\right)\left(r c+\frac{\partial c}{\partial T}\right)} \\
& \geq 0 .
\end{aligned}
$$

The previous proposition shows that an increase in the costs of buying inventory will increase the time in between reordering. This makes intuitive sense, since the earlier a dealer buys new stock, the less time it uses for selling its current stock. And, as can be seen in the proof of Lemma 3.3.8, the lesser time it takes for selling the current stock, the lower its total discounted revenue it receives for will be. So, if costs increase, it will be more fruitful to use some extra time to sell the current stock and, because of the discount factor, pay relatively less for the ordering of new stock.

Proposition 3.4.2. If the dealer plays according to its optimal strategy, an increase in $r$ will change $T^{*}$ as follows:

$$
\begin{aligned}
T_{r}^{* \prime}= & \frac{T^{*} R\left(q\left(T^{*}\right)\right) e^{-2 r T^{*}}-T^{*} c q\left(T^{*}\right)-\frac{1}{r}\left(1-e^{-r T^{*}}\right)\left(e^{-r T^{*}} R\left(q\left(T^{*}\right)\right)-c q\left(T^{*}\right)\right)}{\left(1-e^{-r T^{*}}\right)\left(r c+\frac{\partial c}{\partial T}\right) q\left(T^{*}\right)}(3.31) \\
& +\frac{-\left(1-e^{-r T^{*}}\right) \frac{\partial c}{\partial r} q\left(T^{*}\right)+r e^{-r T^{*}} \int_{0}^{T^{*}} t e^{-r t} R(q(t)) d t-r e^{-r T} \int_{0}^{T} e^{-r t} \frac{d R}{d q} \frac{\partial q(t)}{\partial r} d t}{\left(1-e^{-r T^{*}}\right)\left(r c+\frac{\partial c}{\partial T}\right) q\left(T^{*}\right)} .
\end{aligned}
$$

Proof See appendix.

The complexity of this expression makes it difficult to assess its sign in general. However, with this expression we are able to determine the sign of the derivative $T_{r}^{* \prime}$ for a specific demand function and variables $K, r$ and $S$. This is shown in Figure 3.3, for the linear demand function of $D(p)=1-p$.

As can be seen in the picture, $T^{*}$ increases when $r$ increases. Hereby is the relative size of $S$ of bigger influence than the relative size of $r$. When $r$ increases, less revenue can be made on each batch, but also, the costs of buying a new batch decreases. Intuition might suggest that, since an increase in $r$ makes future profits less valuable, the dealer wants to increase its current revenue by increasing its current sales. The disadvantage of this strategy is that speeding its sales decreases its total discounted revenue on the current stock, as is shown in Lemma 3.3.8. And, the earlier it has to buy new stock, the relatively more it pays for ordering new. Apparently, in some 


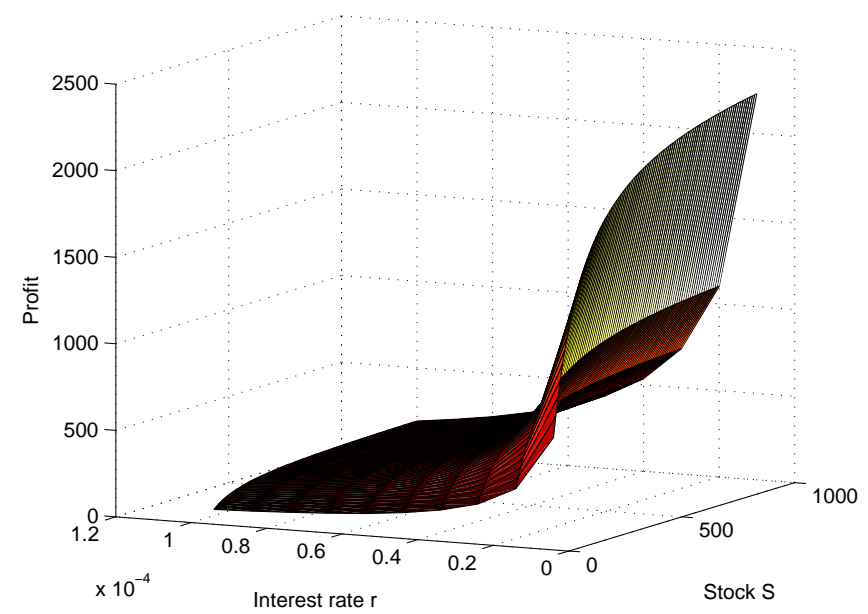

Figure 3.2: The discounted profit of the firm for different interest rates and stock levels, with $K=50$.

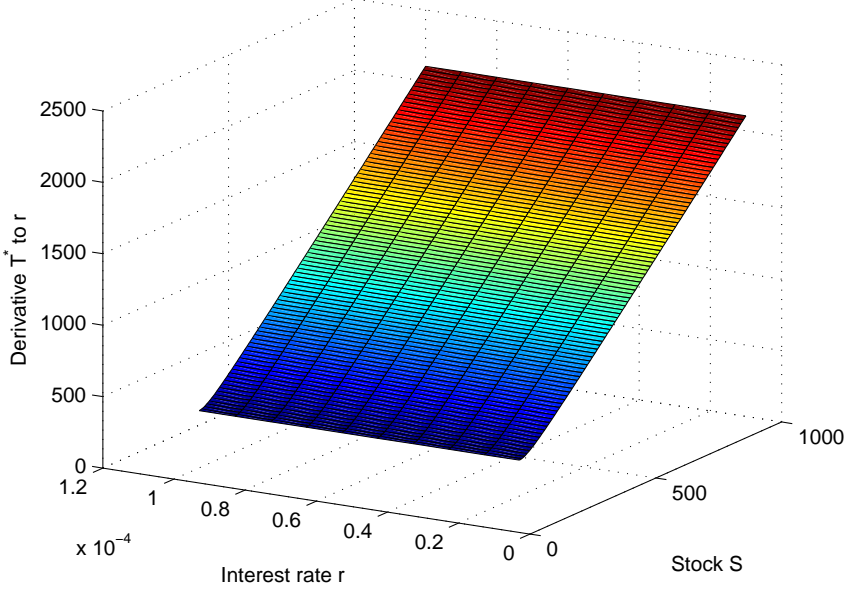

Figure 3.3: The derivative of $T^{*}$ to $r$, when $K=50$. 
situations (and maybe even all), these two factors way heavier on the total discounted sum of profits than the increase in instantaneous revenues does.

Proposition 3.4.3. If the dealer plays according to its optimal strategy, an increase in the stock size will change $T^{*}$ as follows:

$$
T_{S}^{* \prime}=\frac{-\left(1-e^{-r T^{*}}\right) \frac{\partial c}{\partial S} q\left(T^{*}\right)-r e^{-r T^{*}} c}{\left(1-e^{-r T^{*}}\right) q\left(T^{*}\right)\left(\frac{\partial c}{\partial T}+r c\right)} .
$$

Proof See appendix.

Just as with the derivative of $T^{*}$ to $r$, we are unable to determine the sign of the derivative of $T^{*}$ to $S$ for all demand functions. Even though the expression in Proposition 3.4.3 might not look that complex, even for a linear demand function determining the sign is an open problem, if $K, r$ and $S$ are not specified. This is shown in the following example.

Example 3.4.4. Equation (3.29) looks for linear demand of $D(q)=1-q$ as follows:

$$
\frac{1}{4}\left(2 S-T^{*}\right)\left(r\left(2 S-T^{*}\right)+2\right)+\frac{r e^{-r T^{*}}\left(2 S-T^{*}\right)^{2}}{2\left(1-e^{-r T^{*}}\right)}+K=0 .
$$

From this expression, although it determines $T^{*}$ uniquely, it is hard to get an explicit expression for $T^{*}$. To see now if $T^{*}$ increases when $S$ increases, we need to check whether the numerator of (3.32) is strictly bigger than zero, that is, $-\left(1-e^{-r T^{*}}\right) \frac{\partial c}{\partial S} q\left(T^{*}\right)-r e^{-r T^{*}} c \geq 0$. Substituting $R(q)$ for $q(1-q)$ and rewriting, this equation is true iff

$$
\left(1+e^{r T^{*}}\right) \frac{r\left(2 S-T^{*}\right)}{1-e^{r T^{*}}} \leq 1 .
$$

Unfortunately, since we cannot substitute $T^{*}$, we need another way to check whether this holds. Even though we do know that $\frac{e^{r T^{*}} r\left(2 S-T^{*}\right)}{1-e^{r T^{*}}} \leq 1$, from (3.22) and $c^{T} \geq 0$, this is not sufficient to prove (3.33) and we ran out of other useful options to substitute.

An increase of $S$ implies that the dealer has to sell more before it is out of stock. An intuitive consequence of that would be that it takes the dealer longer to sell its stock. We are able to determine, for a specific demand function and variables $K, r$ and $S$, the derivative $T_{S}^{* \prime}$. The values for $T_{S}^{* \prime}$ are shown in Figure 3.4, for the linear demand function of $D(p)=1-p$. As can be seen in the picture in this specific setting, as suspected, $T^{*}$ increases when $S$ increases. 


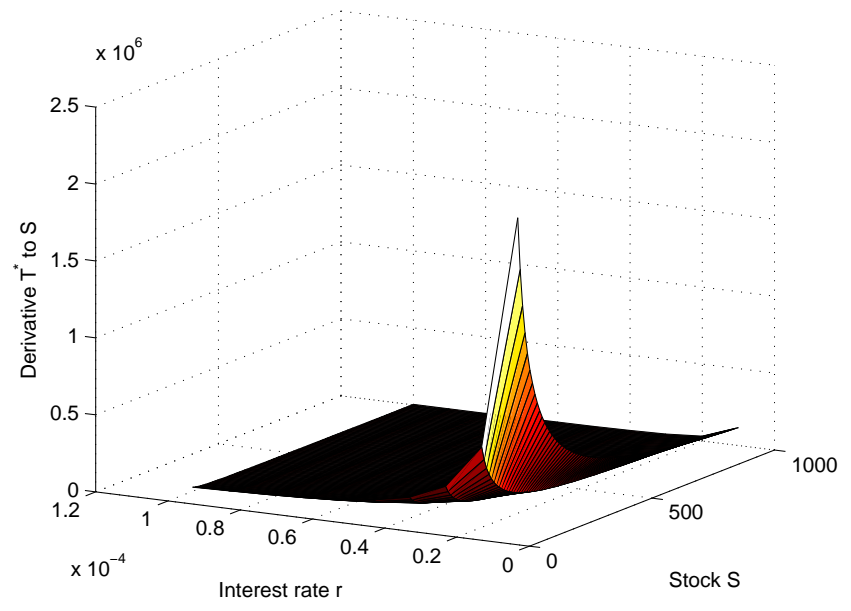

Figure 3.4: The derivative of $T^{*}$ to $S$, when $K=50$.

\subsection{Discussion}

In this chapter, we have shown that there exists a unique strategy that maximizes the total sum of discounted profits. In this strategy, the dealer always reorders after the same amount of time has elapsed. It puts the largest quantity on the market the moment it starts selling its new stock. This quantity continuously decreases until it is at its lowest point right before it runs out of its current stock. This whole cycle repeats itself when it buys new stock again.

A possible way to extend this model would be to add inventory costs to it. Since the size of the batch is fixed, this will not change the amount ordered by the dealer. Making holding inventory costly, will most likely result into the dealer trying to get rid of its stock more quickly. This effect will probably be the strongest right after it has received the new batch, since inventory costs are the highest at that point. Therefore, we conjecture that such an addition will magnify the difference in the quantity setting we have found in this chapter, but will not drastically influence the patterns we have found.

In this chapter it is assumed that the costs of buying new stock are fixed. The production costs of the manufacturer, however, might change over time. For instance, the price of the raw materials might change, or the production 
process itself could become more efficient. If the manufacturer bases its batch price (partly) on these production costs, the dealer needs to take this into account when deciding upon the best moment to reorder. A possible way to extend this model in that direction would be using a trend to predict $K$. 


\section{A Appendix}

\section{Proof of Proposition 3.3.6 b:}

$\frac{\partial q^{T}(t)}{\partial t}:$

To find out how $q^{T}(t)$ changes when $t$ changes, we derive (3.19) to $t$.

$$
\begin{aligned}
\frac{d}{d t} R^{\prime}\left(q^{T}(t)\right) & =R^{\prime \prime}\left(q^{T}(t)\right) \frac{d q^{T}(t)}{d t} \\
& =r c^{T} e^{r t} .
\end{aligned}
$$

Since $R(q)$ is a concave function and $c^{*}$ is positive, this implies that

$$
\frac{d q^{T}(t)}{d t} \leq 0
$$

Note that $\frac{d q^{T}(t)}{d t}=\frac{\partial q^{T}(t)}{\partial t}$.

$$
\frac{\partial q^{T}(t)}{\partial T}:
$$

To see how $q^{T}(t)$ changes when $T$ changes, we derive (3.20) to $T$. Note that $S$ is a constant and therefore

$$
\begin{aligned}
0 & =\frac{d}{d T} \int_{0}^{T} q^{T}(t) d t \\
& =q^{T}(T)+\int_{0}^{T} \frac{d q^{T}(t)}{d T} d t
\end{aligned}
$$

Since $q^{T}(T)>0$, it must hold that $\int_{0}^{T} \frac{d q^{T}(t)}{d T} d t \leq 0$. However, we don't know yet whether this implies that $\frac{d q^{T}(t)}{d T} \leq 0$ for all $t \in[0, T]$. Note that $\frac{\partial q^{T}(t)}{\partial T}=$ $\frac{\partial q^{T}(t)}{\partial T}$. Further information about how $q$ changes with $T$ we get from analyzing how $c^{T}$ changes with $T$.

$\frac{\partial c^{*}}{\partial T}:$

To see how $c^{*}$ changes with $T$, we derive (3.19) to $T$.

$$
\begin{aligned}
\frac{d}{d T} R^{\prime}\left(q^{T}(t)\right) & =\frac{d q^{T}(t)}{d T} R^{\prime \prime}\left(q^{T}(t)\right) \\
& =e^{r t} \frac{d c^{T}}{d T} .
\end{aligned}
$$


Note that, since $\frac{d c^{T}}{d T}$ is independent of $t$ and $e^{r t}>0$ for all $t \in[0, T], e^{r t} \frac{d c^{T}}{d T}$ must have the same sign for all $t \in[0, T]$. This implies that $\frac{d q^{T}}{d T} R^{\prime \prime}\left(q^{T}(t)\right)$ must have the same sign for all $t \in[0, T]$ as well. $R^{\prime \prime}\left(q^{T}(t)\right)<0$ and $\frac{d q^{T}(t)}{d T} \leq 0$ for some $t$ by (3.35). Since $\frac{d q^{T}}{d T} R^{\prime \prime}\left(q^{T}(t)\right)$ has the same sign for all $t \in[0, T]$, it must hold that $\frac{d q^{T}(t)}{d T} \leq 0$ for all $t \in[0, T]$. This automatically implies that $\frac{d c^{T}}{d T} \geq 0$. Note that $\frac{d c^{T}}{d T}=\frac{\partial c^{T}}{\partial T}$.

\section{Proof of Theorem 3.3.11:}

This theorem is proven with the help of the following two lemmas.

Lemma 3.A.1. There exists at most one $T^{*}$ that maximizes total discounted profit.

Proof Define

$$
V(T)=\int_{0}^{T} e^{-r t} R\left(q^{T}(t)\right) d t \text { for } T \in\left[T^{m}, \hat{T}\right]
$$

The best ordering moment can be found by maximizing the following function:

$$
\begin{aligned}
\Pi\left(T^{*}\right) & =\sum_{i=0}^{\infty} e^{-r T^{*} i}\left(V\left(T^{*}\right)-K\right) \\
& =\frac{V\left(T^{*}\right)-K}{\left(1-e^{-r T^{*}}\right)}
\end{aligned}
$$

We get

$$
\begin{aligned}
\frac{d \Pi\left(T^{*}\right)}{d T} & =\frac{V^{\prime}\left(T^{*}\right)\left(1-e^{-r T^{*}}\right)-r e^{-r T^{*}}\left(V\left(T^{*}\right)-K\right)}{\left(1-e^{-r T^{*}}\right)^{2}} \\
& =\frac{\left(1-e^{-r T^{*}}\right)\left(e^{-r T^{*}} R\left(q^{T^{*}}\left(T^{*}\right)\right)-c^{T^{*}} q^{T^{*}}\left(T^{*}\right)\right)+r e^{-r T^{*}}\left(K-\int_{0}^{T^{*}} e^{-r t} R\left(q^{T^{*}}(t)\right) d t\right)}{\left(1-e^{-r T^{*}}\right)^{2}} \\
& =0 .
\end{aligned}
$$

The second equality follows from the first by (3.26). The second derivative of 
$\Pi\left(T^{*}\right)$ is:

$$
\begin{aligned}
\frac{d^{2} \Pi\left(T^{*}\right)}{d T^{2}}= & \left(\frac{r e^{-r T^{*}}\left(e^{-r T^{*}} R\left(q^{T^{*}}\left(T^{*}\right)\right)-c^{T^{*}} q^{T^{*}}\left(T^{*}\right)\right)}{\left(1-e^{-r T^{*}}\right)^{4}}\right. \\
& +\frac{\left(1-e^{-r T^{*}}\right)\left(-r e^{-r T^{*}} R\left(q^{T^{*}}\left(T^{*}\right)\right)+e^{-r T^{*}} \frac{d R}{d q} \frac{d q^{T^{*}}\left(T^{*}\right)}{d T}\right)}{\left(1-e^{-r T^{*}}\right)^{4}} \\
& \frac{\left(1-e^{-r T^{*}}\right)\left(-\frac{d c^{T^{*}}}{d T} q^{T^{*}}\left(T^{*}\right)-c^{T^{*}} \frac{d q^{T^{*}}\left(T^{*}\right)}{d T}\right)}{\left(1-e^{-r T^{*}}\right)^{4}} \\
& +\frac{-r^{2} e^{-r T^{*}}\left(K-\int_{0}^{T^{*}} e^{-r t} R\left(q^{T^{*}}(t)\right) d t\right)}{\left(1-e^{-r T^{*}}\right)^{4}} \\
& \left.\frac{-r e^{-r T^{*}} \int_{0}^{T^{*}} e^{-r t} \frac{d R}{d q} \frac{d q^{T^{*}}(t)}{d T} d t-r e^{-2 r T^{*}} R\left(q^{T^{*}}\left(T^{*}\right)\right)}{\left(1-e^{-r T^{*}}\right)^{4}}\right)\left(1-e^{-r T^{*}}\right)^{2} \\
& -2\left(1-e^{-r T^{*}}\right) r e^{-r T^{*}}\left(\frac{\left(1-e^{-r T^{*}}\right)\left(e^{-r T^{*}} R\left(q^{T^{*}}\left(T^{*}\right)\right)-c^{T^{*}} q^{T^{*}}\left(T^{*}\right)\right)}{\left(1-e^{\left.-r T^{*}\right)^{4}}\right.}\right. \\
& \left.+\frac{r e^{-r T^{*}}\left(K-\int_{0}^{T^{*}} e^{-r t} R\left(q^{T^{*}}(t)\right) d t\right)}{\left(1-e^{-r T^{*}}\right)^{4}}\right) .
\end{aligned}
$$

To see whether we have a local maximum or minimum at $\frac{d \Pi\left(T^{*}\right)}{d T}=0$, we substitute the expression for $\frac{d \Pi\left(T^{*}\right)}{d T}$ in the expression for $\frac{d^{2} \Pi\left(T^{*}\right)}{d T^{2}}$. Since we only want to know the sign of this expression, and the denominator is always positive, we can leave the dominator out. So, the sign of $\frac{d^{2} \Pi\left(T^{*}\right)}{d T^{2}}$ at $\frac{d \Pi\left(T^{*}\right)}{d T}=0$ is

$$
\begin{aligned}
& r e^{-r T^{*}}\left(e^{-r T^{*}} R\left(q^{T^{*}}\left(T^{*}\right)\right)-c^{T^{*}} q^{T^{*}}\left(T^{*}\right)\right)+\left(1-e^{-r T^{*}}\right)\left(-r e^{-r T^{*}} R\left(q^{T^{*}}\left(T^{*}\right)\right)\right. \\
& \left.+e^{-r T^{*}} \frac{d R}{d q} \frac{d q^{T^{*}}\left(T^{*}\right)}{d T}-\frac{d c^{T^{*}}}{d T} q^{T^{*}}\left(T^{*}\right)-c^{T^{*}} \frac{d q^{T^{*}}\left(T^{*}\right)}{d T}\right) \\
& -r^{2} e^{-r T^{*}}\left(K-\int_{0}^{T^{*}} e^{-r t} R\left(q^{T^{*}}(t)\right) d t\right)-r e^{-2 r T^{*}} R\left(q^{T^{*}}\left(T^{*}\right)\right) \\
& -r e^{-r T^{*}} \int_{0}^{T^{*}} e^{-r t} \frac{d R}{d q} \frac{d q^{T^{*}}(t)}{d T} d t
\end{aligned}
$$




$$
\begin{aligned}
& =r e^{-r T^{*}}\left(e^{-r T^{*}} R\left(q^{T^{*}}\left(T^{*}\right)\right)-c^{T^{*}} q^{T^{*}}\left(T^{*}\right)\right)+\left(1-e^{-r T^{*}}\right)\left(-r e^{-r T^{*}} R\left(q^{T^{*}}\left(T^{*}\right)\right)\right. \\
& \left.+e^{-r T^{*}} \frac{d R}{d q} \frac{d q^{T^{*}}\left(T^{*}\right)}{d T}-\frac{d c^{T^{*}}}{d T} q^{T^{*}}\left(T^{*}\right)-c^{T^{*}} \frac{d q^{T^{*}}\left(T^{*}\right)}{d T}\right) \\
& +r\left(1-e^{-r T^{*}}\right)\left(e^{-r T^{*}} R\left(q^{T^{*}}\left(T^{*}\right)\right)-c^{T^{*}} q^{T^{*}}\left(T^{*}\right)\right) \\
& -r e^{-2 r T^{*}} R\left(q^{T^{*}}\left(T^{*}\right)\right)-r e^{-r T^{*}} \int_{0}^{T^{*}} e^{-r t} \frac{d R}{d q} \frac{d q^{T^{*}}(t)}{d T} d t \\
& =r e^{-r T^{*}}\left(e^{-r T^{*}} R\left(q^{T^{*}}\left(T^{*}\right)\right)-c^{T^{*}} q^{T^{*}}\left(T^{*}\right)\right) \\
& +\left(1-e^{-r T^{*}}\right)\left(-r e^{-r T^{*}} R\left(q^{T^{*}}\left(T^{*}\right)\right)-\frac{d c^{T^{*}}}{d T} q^{T^{*}}\left(T^{*}\right)\right) \\
& +r\left(1-e^{-r T^{*}}\right)\left(e^{-r T^{*}} R\left(q^{T^{*}}\left(T^{*}\right)\right)-c^{T^{*}} q^{T^{*}}\left(T^{*}\right)\right) \\
& -r e^{-2 r T^{*}} R\left(q^{T^{*}}\left(T^{*}\right)\right)-r e^{-r T^{*}} \int_{0}^{T^{*}} e^{-r t} \frac{d R}{d q} \frac{\partial q}{\partial T} d t \\
& =-\left(1-e^{-r T^{*}}\right)\left(r e^{-r T^{*}} R\left(q^{T^{*}}\left(T^{*}\right)\right)+\frac{d c^{T^{*}}}{d T} q^{T^{*}}\left(T^{*}\right)\right) \\
& +r\left(e^{-r T^{*}} R\left(q^{T^{*}}\left(T^{*}\right)\right)-c^{T^{*}} q^{T^{*}}\left(T^{*}\right)\right) \\
& -r e^{-2 r T^{*}} R\left(q^{T^{*}}\left(T^{*}\right)\right)-r e^{-r T^{*}} \int_{0}^{T^{*}} e^{-r t} \frac{d R}{d q} \frac{d q^{T^{*}}(t)}{d T} d t \\
& =-\left(1-e^{-r T^{*}}\right)\left(r e^{-r T^{*}} R\left(q^{T^{*}}\left(T^{*}\right)\right)+\frac{d c^{T^{*}}}{d T} q^{T^{*}}\left(T^{*}\right)\right) \\
& +r\left(e^{-r T^{*}} R\left(q^{T^{*}}\left(T^{*}\right)\right)-c^{T^{*}} q^{T^{*}}\left(T^{*}\right)\right) \\
& -r e^{-2 r T^{*}} R\left(q^{T^{*}}\left(T^{*}\right)\right)-r e^{-r T^{*}} \int_{0}^{T^{*}} c^{T^{*}} \frac{d q^{T^{*}}(t)}{d T} d t \\
& =-\left(1-e^{-r T^{*}}\right)\left(r e^{-r T^{*}} R\left(q^{T^{*}}\left(T^{*}\right)\right)+\frac{d c^{T^{*}}}{d T} q^{T^{*}}\left(T^{*}\right)\right) \\
& +r\left(e^{-r T^{*}} R\left(q^{T^{*}}\left(T^{*}\right)\right)-c^{T^{*}} q^{T^{*}}\left(T^{*}\right)\right) \\
& -r e^{-2 r T^{*}} R\left(q^{T^{*}}\left(T^{*}\right)\right)+r e^{-r T^{*}} c^{T^{*}} q^{T^{*}}\left(T^{*}\right) \\
& =-\left(1-e^{-r T^{*}}\right) q^{T^{*}}\left(T^{*}\right) \frac{d c^{T^{*}}}{d T}-r q^{T^{*}}\left(T^{*}\right) c^{T^{*}}+r e^{-r T^{*}} c^{T^{*}} q^{T^{*}}\left(T^{*}\right) \\
& =-\left(1-e^{-r T^{*}}\right) q^{T^{*}}\left(T^{*}\right)\left(\frac{d c^{T^{*}}}{d T}+r c^{T^{*}}\right) \\
& \leq 0 \text {. }
\end{aligned}
$$

Here, the first equality follows from (3.38), the second from (3.19), the third from rewriting, the fourth from (3.19) again, the fifth from (3.36), and the re- 
maining follow from rewriting again. Since $\frac{d c^{T^{*}}}{d T^{*}}$ and $r c^{T^{*}}$ are both positive, the inequality follows.

Given that the second derivative is always negative for a stationary point, we know that there can only be on such a point.

Lemma 3.A.2. There exists at least one $T^{*}$ that maximizes total discounted profit.

Proof If we can show that the first derivative is positive for small $T^{*}$ and negative for large $T^{*}$, a local maximum exists and is also the global maximum.

The minimum number of time it will take for a dealer to sell its batch $S$ is $T^{m}=S / q^{m}$, and in this situation, $c^{T^{*}}=0$ and $q^{T^{*}}(t)=q^{m}$ for every $t \in \mathbb{R}^{+}$. At this point,

$$
\begin{aligned}
\frac{d \Pi\left(T^{m}\right)}{d T} & =\frac{\left(1-e^{-r T^{m}}\right) R\left(q^{m}\right) e^{-r T^{m}}+r e^{-r T^{m}}\left(K-\int_{0}^{T^{m}} e^{-r t} R\left(q^{m}\right) d t\right)}{\left(1-e^{-r T^{m}}\right)^{2}} \\
& =\frac{\left(1-e^{-r T^{m}}\right) R\left(q^{m}\right) e^{-r T^{m}}+r e^{-r T^{m}} K-r e^{-r T^{m}}\left[-\frac{1}{r} e^{-r t} R\left(q^{m}\right)\right]_{0}^{T^{m}}}{\left(1-e^{-r T^{m}}\right)^{2}} \\
& =\frac{r e^{-r T^{m}} K}{\left(1-e^{-r T^{m}}\right)^{2}} \\
& \geq 0 .
\end{aligned}
$$

So, for small $T^{*}$, profit is increasing with $T^{*}$. We know that the largest $T^{*}$ can be is where $q^{T^{*}}\left(T^{*}\right)=0$, this gives:

$$
\begin{aligned}
\frac{d \Pi\left(T^{*}\right)}{d T} & =\frac{\left(1-e^{-r T^{*}}\right) e^{-r T^{*}} R(0)+r e^{-r T^{*}}\left(K-\int_{0}^{T^{*}} e^{-r t} R\left(q^{T^{*}}(t)\right) d t\right)}{\left(1-e^{-r T^{*}}\right)^{2}} \\
& =\frac{r e^{-r T^{*}}\left(K-\int_{0}^{T^{*}} e^{-r t} R\left(q^{T^{*}}(t)\right) d t\right)}{\left(1-e^{-r T^{*}}\right)^{2}} \\
& \leq 0 .
\end{aligned}
$$

From our assumption that $K<\max \int_{0}^{T^{*}} e^{-r t} R\left(q^{T^{*}}(t)\right) d t$ it follows that this derivative is negative.

In Lemma 3.A. 1 and 3.A.2, it is shown that there is at most one $T^{*}$ and at least one $T^{*}$ that maximizes total discounted profit. Ergo, there is a unique $T^{*}$ that maximizes total discounted profit. This $T^{*}$ is found by solving the first derivative of $\Pi(T)$ (equation (3.38)) to zero. That is, $T^{*}$ solves 
$\frac{\left(1-e^{-r T^{*}}\right)\left(e^{-r T^{*}} R\left(q^{T^{*}}\left(T^{*}\right)\right)-c^{T^{*}} q^{T^{*}}\left(T^{*}\right)\right)+r e^{-r T^{*}}\left(K-\int_{0}^{T^{*}} e^{-r t} R\left(q^{T^{*}}(t)\right) d t\right)}{\left(1-e^{-r T^{*}}\right)^{2}}=0$.

\section{Proof of Proposition 3.4.1:}

Proof To see how $T^{*}$ changes when $K$ changes, we first need to make $T^{*}$ a function of $K$ and derive to $K$. We now that

$$
\begin{aligned}
\frac{d \Pi\left(T^{*}\right)}{d T} & =\frac{\left(1-e^{-r T^{*}}\right)\left(e^{-r T^{*}} R\left(q\left(T^{*}\right)\right)-c q\left(T^{*}\right)\right)+r e^{-r T^{*}}\left(K-\int_{0}^{T^{*}} e^{-r t} R(q(t)) d t\right)}{\left(1-e^{-r T^{*}}\right)^{2}} \\
& =0 .
\end{aligned}
$$

Note that $q$ is influenced by a change in $K$, directly via $T$ and even more if also $t=T$. Therefore, the optimum $T^{*}$ always has to solve

$$
\begin{aligned}
M(K)= & \left(1-e^{-r T^{*}}\right)\left(e^{-r T^{*}} R\left(q\left(T^{*}\right)\right)-c q\left(T^{*}\right)\right) \\
& +r e^{-r T^{*}}\left(K-\int_{0}^{T^{*}} e^{-r t} R(q(t)) d t\right) \\
= & 0 .
\end{aligned}
$$

The derivative to $K$ of $M(K)$ should still be equal to zero and is the following.

$$
\begin{aligned}
\frac{d M(K)}{d K} & =-T_{K}^{* \prime}\left(1-e^{-r T^{*}}\right) q\left(T^{*}\right)\left(r c+\frac{\partial c}{\partial T}\right)+r e^{-r T^{*}} \\
& =0
\end{aligned}
$$

Here, the equality follows directly from (3.46) and deriving $M(K)$ to the separate variable $K$. Therefore,

$$
\begin{aligned}
T_{K}^{* \prime} & =\frac{r e^{-r T^{*}}}{\left(1-e^{-r T^{*}}\right) q\left(T^{*}\right)\left(r c+\frac{\partial c}{\partial T}\right)} \\
& \geq 0 .
\end{aligned}
$$




\section{Proof of Proposition 3.4.2:}

Proof To see how $T^{*}$ changes when $r$ changes, we first have to note that $T^{*}$ is not only directly influenced by $r$, but also via $c$ and $q$, as can be seen in Proposition 3.3.4. So, if $r$ is no longer a constant, but a variable, then $c, q$ and $T^{*}$ all become functions of $r$. Now, (3.29) becomes

$$
\begin{aligned}
M(r)= & \left(1-e^{-r T^{*}}\right)\left(e^{-r T^{*}} R\left(q\left(T^{*}\right)\right)-c q\left(T^{*}\right)\right) \\
& +r e^{-r T^{*}}\left(K-\int_{0}^{T^{*}} e^{-r t} R(q(t)) d t\right) \\
= & 0 .
\end{aligned}
$$

When deriving this function to $r$, we get the following.

$$
\begin{aligned}
\frac{d M(r)}{d r}= & -T_{r}^{* \prime}\left(1-e^{-r T^{*}}\right) q\left(T^{*}\right)\left(r c+\frac{\partial c}{\partial T}\right)+T^{*} R\left(q\left(T^{*}\right)\right) e^{-2 r T^{*}}-T^{*} c q\left(T^{*}\right) \\
& -\frac{1}{r}\left(1-e^{-r T^{*}}\right)\left(e^{-r T^{*}} R\left(q\left(T^{*}\right)\right)-c q\left(T^{*}\right)\right)-\left(1-e^{-r T^{*}}\right) \frac{\partial c}{\partial r} q\left(T^{*}\right) \\
& +r e^{-r T^{*}} \int_{0}^{T^{*}} t e^{-r t} R(q(t)) d t-r e^{-r T} \int_{0}^{T} e^{-r t} \frac{d R}{d q} \frac{\partial q(t)}{\partial r} d t \\
= & 0 .
\end{aligned}
$$

Therefore,

$$
\begin{aligned}
T_{r}^{* \prime}= & \frac{T^{*} R\left(q\left(T^{*}\right)\right) e^{-2 r T^{*}}-T^{*} c q\left(T^{*}\right)-\frac{1}{r}\left(1-e^{-r T^{*}}\right)\left(e^{-r T^{*}} R\left(q\left(T^{*}\right)\right)-c q\left(T^{*}\right)\right)}{\left(1-e^{-r T^{*}}\right)\left(r c+\frac{\partial c}{\partial T}\right) q\left(T^{*}\right)} \\
& +\frac{-\left(1-e^{-r T^{*}}\right) \frac{\partial c}{\partial r} q\left(T^{*}\right)+r e^{-r T^{*}} \int_{0}^{T^{*}} t e^{-r t} R(q(t)) d t-r e^{-r T} \int_{0}^{T} e^{-r t} \frac{d R}{d q} \frac{\partial q(t)}{\partial r} d t}{\left(1-e^{-r T^{*}}\right)\left(r c+\frac{\partial c}{\partial T}\right) q\left(T^{*}\right)} .
\end{aligned}
$$

\section{Proof of Proposition 3.4.3:}

Proof To see how $T^{*}$ changes when $S$ changes, observe that $S$ is not directly part of equation (3.29). However, a change in $S$ will change (3.20) and therefore (3.19). Since $T$ is constant here, the influence of a change in $S$ will be captured by a change of $q(t)$ in (3.20). Because $q(t)$ changes with $S$, it 
can be seen in (3.19) that therefore also $c$ changes with $S$. These equations change as follows when $S$ changes:

$$
\frac{d}{d S} \int_{0}^{T} q(t) d t=\int_{0}^{T} \frac{\partial q(t)}{\partial S} d t
$$

and

$$
\begin{aligned}
\frac{d}{d S} R^{\prime}(q(t)) & =R^{\prime \prime}(q(t)) \frac{\partial q(t)}{\partial S} \\
& =\frac{\partial c}{\partial S} e^{r t}
\end{aligned}
$$

Since $c$ doesn't depend on $t$, the sign of (3.49) must be the same for all $t \in$ $[0, T]$. Therefore, the sign of (3.48) must be the same for all $t \in[0, T]$ as well. Equation (3.47) tells us that there must be some $t \in[0, T]$ for which it holds that $\frac{\partial q(t)}{\partial S}>0$. This implies that $\frac{\partial q(t)}{\partial S} \geq 0$ for all $t \in[0, T]$ and since $R^{\prime \prime}(q(t))<0, \frac{\partial c}{\partial S} \leq 0$.

To see now how $T^{*}$ actually changes with $S$, we need the expression for the optimal $T^{*}$ again. Let

$$
\begin{aligned}
M(S)= & \left(1-e^{-r T^{*}}\right)\left(e^{-r T^{*}} R\left(q\left(T^{*}\right)\right)-c q\left(T^{*}\right)\right) \\
& +r e^{-r T^{*}}\left(K-\int_{0}^{T^{*}} e^{-r t} R(q(t)) d t\right) \\
= & 0 .
\end{aligned}
$$


Then

$$
\begin{aligned}
\frac{\partial M}{\partial S}= & -T_{S}^{* \prime}\left[\left(1-e^{-r T^{*}}\right)\left(r c+\frac{\partial c}{\partial T}\right) q\left(T^{*}\right)\right]+ \\
& \left(1-e^{-r T^{*}}\right)\left(e^{-r T^{*}} R^{\prime}\left(q\left(T^{*}\right)\right) \frac{\partial q\left(T^{*}\right)}{\partial S}-\frac{\partial c}{\partial S} q\left(T^{*}\right)-c \frac{\partial q\left(T^{*}\right)}{\partial S}\right) \\
& -r e^{-r T^{*}} \int_{0}^{T^{*}} e^{-r t} \frac{\partial q(t)}{\partial S} R^{\prime}(q(t)) d t \\
= & -T_{S}^{* \prime}\left(1-e^{-r T^{*}}\right) q\left(T^{*}\right)\left(\frac{\partial c}{\partial T}+r c\right)+\left(1-e^{-r T^{*}}\right)\left(c \frac{\partial q\left(T^{*}\right)}{\partial S}\right. \\
& \left.-\frac{\partial c}{\partial S} q\left(T^{*}\right)-c \frac{\partial q\left(T^{*}\right)}{\partial S}\right)-r e^{-r T^{*}} \int_{0}^{T^{*}} \frac{\partial q(t)}{\partial S} c d t \\
= & -T_{S}^{* \prime}\left(1-e^{-r T^{*}}\right) q\left(T^{*}\right)\left(\frac{\partial c}{\partial T}+r c\right)-\left(1-e^{-r T^{*}}\right) \frac{\partial c}{\partial S} q\left(T^{*}\right) \\
& -r e^{-r T^{*}} c \int_{0}^{T^{*}} \frac{\partial q(t)}{\partial S} d t \\
= & -T_{S}^{* \prime}\left(1-e^{-r T^{*}}\right) q\left(T^{*}\right)\left(\frac{\partial c}{\partial T}+r c\right)-\left(1-e^{-r T^{*}}\right) \frac{\partial c}{\partial S} q\left(T^{*}\right)-r e^{-r T^{*}} c \\
= & 0 .
\end{aligned}
$$

Here, the second equality sign follows from (3.19), the third from moving the constant $c$ out of the integral and the fourth from (3.47). Therefore,

$$
T_{S}^{* \prime}=\frac{-\left(1-e^{-r T^{*}}\right) \frac{\partial c}{\partial S} q\left(T^{*}\right)-r e^{-r T^{*}} c}{\left(1-e^{-r T^{*}}\right) q\left(T^{*}\right)\left(\frac{\partial c}{\partial T}+r c\right)} .
$$





\section{Chapter 4}

\section{Collusion in a Price-Quantity Oligopoly}

\subsection{Introduction}

"The Cournot story concerns producers who simultaneously and independently make production quantity decisions, and who then bring what they have produced to the market, with the market price being the price that equates the total supply with demand. The Bertrand story, on the other hand, concerns producers who simultaneously and independently name prices. Demand is allocated to the low-price producer(s), who then produce (up to) the demand they encounter ... There are two differences in these stories: how price is determined (by an auctioneer in Cournot and by price "competition" in Bertrand), and when production is supposed to take place." (Kreps and Scheinkman (1983), p. 326)

Both the Cournot and the Bertrand story have significantly enhanced our understanding of strategic firm behavior in oligopolies. Yet, both have also been subject to severe criticism. For instance, Cournot's model requires an auctioneer to determine the market price, whereas in practice prices are typically set by suppliers themselves. In this respect, Bertrand's story is more satisfactory. However, this model assumes that production follows the realization of demand, whereas in a great many industries production takes place in advance of sale. More generally, and independent of the timing of 
production, it seems more reasonable to assume that firms choose both their prices and production levels. This is true when producers compete, but no different when they collude.

The objective of this chapter is to study (optimal) collusion among firms that have both price and quantity as a strategic choice variable. ${ }^{1}$ Towards that end, we analyze an infinitely repeated oligopoly game in which firms simultaneously make both price and production decisions in each period. This mode of production we will call production in advance. With production in advance, production costs are incurred before a firm learns how much it will actually sell. At the end of this chapter, we also shortly analyze what happens when firms produce to order. When production is to order, producers choose a price and produce after their private demand is known. Thus, the key difference between both settings lies in whether or not firms commit to a particular production level before the realization of demand. We therefore incorporate the Cournot and Bertrand story regarding the timing of production, but price decisions are made by the producers themselves (i.e., there is no auctioneer).

In attempting to combine the more plausible elements of both stories, existing literature has primarily focused on settings in which firms first choose production capacities and then engage in price competition. The reason for analyzing price competition given capacities is that prices can presumably be adjusted more quickly than quantity-related variables. For example, Kreps and Scheinkman (1983) find that Bertrand competition may yield Cournot outcomes when suppliers first choose a scale of operation. As to collusion, Fershtman and Muller (1986) explore the impact of long-run competition in capital investments on collusion on prices and market shares and show, among other things, that competition in capacities has no destabilizing effect on collusion. Benoit and Krishna (1987) show that when firms are allowed to collude on both price and capacity then all collusive equilibria have firms holding excess capacity. Davidson and Deneckere (1990) establish that more collusion requires higher levels of excess capacity. Holding excess capacity is required to enforce a collusive scheme as it allows to punish a deviator by increasing production. In the current chapter, we assume that firms adopt grim-trigger strategies to sustain collusion and that there is sufficient production capacity to implement this punishment strategy. Consequently, our focus is on the actual quantity decisions and not on the maximum amount of products a firm can offer each period. Our model

${ }^{1}$ This chapter is based on the paper van den Berg and Bos (2011). 
of production in advance falls in the class of games described by Maskin (1986). He claims that an equilibrium exists in the static game, but refrains from verifying the conditions as given by Dasgupta and Maskin (1986) for the oligopolistic setting. Next to this, collusion is not a part of the paper.

To our knowledge, we are the first to study collusion in a price-quantity oligopoly while taking account of different timing of production. ${ }^{2}$ As suppliers have control over both price and quantity, one question of interest is what choice variables they will optimally collude on. Will they choose to collude on either price or quantity (semicollusion) or both (full collusion)? ${ }^{3}$ In this chapter, we restrict attention to price collusion and price-quantity collusion. Put differently, firms are given the possibility to either form a price-fixing cartel or a price-quota cartel. ${ }^{4}$ Obviously, since firms can imitate semicollusion by colluding in full, full collusion always gives the suppliers at least as much profit as colluding on only one variable will. However, there are at least three reasons why firms may prefer to collude on one variable only after all. First, reaching consensus on coordination of an additional variable complicates the bargaining process and there is plenty of evidence from practice that bargaining issues should not be take too lightly. For example, Levenstein and Suslow (2004) state that "Bargaining problems were much more likely to undermine collusion than was secret cheating. About one quarter of the cartel episodes ended because of bargaining problems. Bargaining issues affected virtually every industry studied." Second, coordinating on more variables may complicate enforcement as more monitoring is required to ensure compliance. Third, more complete cartel contracts leave additional traces of evidence and therefore, ceteris paribus, increase the probability of a conviction. In short, whenever a price cartel is more or less equally successful as a price-quota cartel, firms will arguably prefer not to install an additional quota agreement. For both modes of production, we first analyze optimal price collusion and then evaluate the potential additional value of a quota agreement.

The analysis in this study thus sheds some light on one particular type of cartel heterogeneity: why do some cartels entail an agreement on both prices and quantities, whereas others include only an arrangement on prices? For

\footnotetext{
${ }^{2}$ Contributions that consider competition in price-quantity oligopolies include, for example, Kreps and Scheinkman (1983), Friedman (1988), Dixon (1992) and Tasnádi (2006).

${ }^{3}$ For a recent survey of literature on semicollusion, see Steen and Sørgard (2009).

${ }^{4}$ In this study, whenever we use the term (sales) quota(s), we mean an allocation of quantities or market shares.
} 
example, in Carbonless Paper members agreed on the timing and magnitude of price increases for each EEA country. ${ }^{5}$ However, these price-fixing agreements were only sometimes accompanied by quota agreements (e.g., in Spain and France). In Elevators and Escalators members coordinated prices and allocated projects on the basis of a pre-arranged market share scheme. ${ }^{6}$ In particular, a compensation mechanism was adopted to ensure alignment of overall project value with the allocated shares. Yet, in the Netherlands the cartel operated on a project by project basis seemingly without a clear compensation scheme. In Methionine parties fixed minimum and target prices and agreed on concerted price increases. ${ }^{7}$ One of the members proposed a volume control scheme including a compensation mechanism, but sales quotas were never implemented. There are many more real-world examples of price and price-quota cartels. ${ }^{8}$

We find that firms almost always prefer to install a price-quota cartel when production is in advance of sales. However, the reasons for adopting a market sharing scheme in addition to a price-fixing agreement differ. When market demand is relatively elastic, firms will optimally set the monopoly price. In this case, an arrangement on outputs allows firms to deal with both coordination and incentive problems. ${ }^{9}$ The coordination problem concerns the fact that in equilibrium every member should have a sufficiently high level of sales, while ensuring that the market clears. The incentive problem prescribes the cartel to allocate a sufficiently large part of total sales to the smallest members as they appear to have the strongest incentive to defect from the agreement. Therefore, given that the size distribution of members is sufficiently heterogeneous, a price-quota cartel is ceteris paribus more likely than a price-fixing cartel. If market demand is relatively inelastic, then the coordination problem is absent. The reason being that in this case all members optimally produce identical amounts. Yet, sustainability of collusion requires the cartel to set a price below the monopoly price in order to mitigate incentives to defect. In this case, a price-quota cartel is found to be always more profitable than a price-fixing cartel. Specifically, installing

\footnotetext{
${ }^{5}$ Case COMP/E-1/36.212 - Carbonless paper.

${ }^{6}$ Case COMP/E-1/38.823 - PO/Elevators and Escalators.

${ }^{7}$ Case C.37.519 - Methionine.

${ }^{8}$ See, for instance, Russo et al. (2010). This book provides a complete overview and description of all European cartel cases between 1962 and 2009.

${ }^{9}$ Osborne (1976) identifies four internal problems a cartel may be confronted with. The cartel has to locate the contract surface and choose a point on that surface (the coordination problem). Additionally, it has to detect and deter cheating (the incentive problem).
} 
an optimal market sharing scheme allows firms to avoid overproduction and to sustain the monopoly price.

By contrast, overproduction does not occur in equilibrium when production follows the realization of demand. Moreover, for both price collusion and price-quantity collusion the profit-maximizing cartel price is the monopoly price. In this case, an additional quota agreement is made solely when a price cartel alone is not sustainable. As with production in advance, the smallest members have the strongest incentive to deviate from the cartel agreement. Hence, sustainability of collusion may require a redistribution of sales from the larger to the smaller cartel participants. Overall, our study suggests that a price-quota cartel is likely to be the rule rather than the exception. An additional quota agreement may not have added value when firms produce to order and are more or less of equal size.

We believe one should be reluctant to bring up evidence from antitrust practice to support general theoretical predictions of cartel behavior like ours. One reason for this is that known cartels differed in many respects. Moreover, they have been operating in a wide variety of industries and had to deal with specific problems. As a result, there are potentially many explanations for observed collusive conduct. Apart from this, relevant information that is required to confidently match a general theoretical framework with a specific cartel case is often lacking. However, in this respect the lysine cartel forms a notable exception. This cartel is one of the most well-documented cases in antitrust history and of particular interest to our study as it had two "lives" ${ }^{10}$ During its first life, members fixed prices but no market sharing scheme was adopted. During its second life, members also agreed on a market share allocation. The market for lysine is characterized by homogeneous products, approximately constant unit production costs and relatively inelastic market demand. In this case, our model would predict firms to prefer a price-quota cartel as it allows them to sustain higher prices. This is indeed what happened. That is, the cartel set prices at a significantly higher level during its second life, which was generally more successful. Therefore, there exists some support for our theoretical findings in antitrust practice.

This chapter proceeds as follows. The next section introduces the model. Section 4.3 describes the stationary equilibria in markets with production in advance. In the next section, Section 4.4, the conditions are analyzed under which a firm prefers collusion over competition. Section 4.5 provides an analysis of price- and price-quantity collusion. Section 4.6 describes the

${ }^{10}$ See, for instance, Connor (2001) and De Roos (2006). 
stationary equilibria and an analysis of price- and price-quantity collusion when firms produce to order. Section 4.7 relates our main findings to existing literature that considers private information and demand uncertainty. Section 4.8 concludes. All proofs are relegated to the Appendix.

\subsection{Model}

We consider a homogeneous good industry in which a fixed and finite set of firms, denoted by $N=\{1, \ldots, n\}$, interact. In this section, we will describe our assumptions for the static setting. In Section 4.4, to analyze the possibilities to collude, we extend this model to be repeated an infinite amount of times.

Firms simultaneously make price and production decisions so as to maximize their expected profit. We define $A \equiv[0, a] \times[0, b]$ as the common action set and price and quantity choices are respectively denoted by the vectors $\boldsymbol{p} \equiv\left(p_{1}, \ldots, p_{n}\right)$ and $\boldsymbol{q} \equiv\left(q_{1}, \ldots, q_{n}\right)$, where $p_{i} \in[0, a]$ and $q_{i} \in[0, b]$ for all $i \in$ $N$. Define $\boldsymbol{p}_{-\boldsymbol{i}} \equiv\left(p_{1}, \ldots, p_{i-1}, p_{i+1}, \ldots, p_{n}\right)$ and $\boldsymbol{q}_{-\boldsymbol{i}} \equiv\left(q_{1}, \ldots, q_{i-1}, q_{i+1}, \ldots, q_{n}\right)$ as the vector of respectively prices and quantities of all firms other than $i$. Firms have identical unit production costs $c \in(0, a)$ and sufficient production capacity available (i.e., there are no capacity constraints, $b$ is high enough not to influence the quantity-decision of the firms).

Market demand is given by $D(p)$, which is a continuous, strictly decreasing and concave function of price in the range $[0, a]$. Additionally, we assume that this function is identically zero on $[a, \infty)$, twice continuously differentiable on $(0, a)$ and $D(0)<b .{ }^{11}$ The monopoly price and output are respectively indicated by $p^{m}$ and $q^{m}: D\left(p^{m}\right)+\left(p^{m}-c\right) D^{\prime}\left(p^{m}\right)=0$ and $q^{m}=D\left(p^{m}\right)$. Firm $i$ 's individual demand depends on $\boldsymbol{p}$ and $\boldsymbol{q}$. Consumers buy first from a supplier charging the lowest price. In case of a tie, demand is shared according to ratio. In specifying firm $i$ 's demand, define $\Delta\left(p_{i}\right) \equiv\left\{j \in N: p_{j}<p_{i}\right\}$ and $\Omega\left(p_{i}\right) \equiv\left\{j \in N: p_{j}=p_{i}\right\}$ as the set of firms that respectively price below and at $p_{i} \cdot{ }^{12}$

With production in advance, production takes place before the realiza-

\footnotetext{
${ }^{11}$ We let $b>D(0)$, since - as is described below - demand is shared proportionally when at least one other firm sets the same price. This could give a firm the incentive to produce more than $D(0)$. It can easily be verified that there always exists an upperbound on how much a firm is willing to produce.

${ }^{12}$ Officially, it is $\Delta\left(p_{i}, \mathbf{p}_{-\mathbf{i}}\right)$ and $\Omega\left(p_{i}, \mathbf{p}_{-\mathbf{i}}\right)$. From the context, it will be clear what $\mathbf{p}_{-\mathbf{i}}$ is. Therefore, we use shorthand notation here to stress which value of $p_{i}$ these sets are based on.
} 
tion of demand. ${ }^{13}$ We suppose that in this case demand is established according to a proportional demand allocation rule. Thus, if two or more firms charge the same price and total supply exceeds total demand at that price, then sales are assumed proportional to individual levels of production. The proportion that firm $i$ receives of the (residual) demand at that price, is denoted by $\lambda_{i} \cdot{ }^{14}$

Assumption 4.2.1. Assume production in advance. Then $\lambda_{i}=\frac{q_{i}}{\sum_{j \in \Omega\left(p_{i}\right) q_{j}}}$, for all $i \in N$.

Consequently, suppliers with more products available receive a larger share of market demand, all else unchanged. ${ }^{15}$

Demand for the products of firm $i$ is denoted $D_{i}\left(p_{i}, q_{i}, \mathbf{p}_{-\mathbf{i}}, \mathbf{q}_{-\mathbf{i}}\right)$. For any price-quantity configuration, its profit is thus given by

$$
\pi_{i}\left(p_{i}, q_{i}, \mathbf{p}_{-\mathbf{i}}, \mathbf{q}_{-\mathbf{i}}\right)=p_{i} D_{i}\left(p_{i}, q_{i}, \mathbf{p}_{-\mathbf{i}}, \mathbf{q}_{-\mathbf{i}}\right)-c q_{i} .
$$

Firm $i$ 's demand is then of the following general structure:

$$
D_{i}\left(p_{i}, q_{i}, \mathbf{p}_{-\mathbf{i}}, \mathbf{q}_{-\mathbf{i}}\right)=\min \left\{q_{i}, \lambda_{i}\left(D\left(p_{i}\right)-\sum_{k \in \Delta\left(p_{i}\right)} q_{k}\right)^{+}\right\} .
$$

\subsection{Static Nash Equilibrium}

In this section, we characterize the single-shot Nash equilibrium of the model described above. When production is in advance of sales, there is no purestrategy Nash equilibrium. However, there does exist a symmetric mixedstrategy Nash equilibrium for which it can be shown that expected profits amount to zero.

Suppose that production is in advance of sales. The next result establishes that in this case there exists no pure-strategy Nash equilibrium. Yet, we can apply Corollary 5.3 of Reny (1999) to establish the existence of a symmetric mixed-strategy Nash equilibrium.

\footnotetext{
${ }^{13}$ That is, a firm only learns about the production levels and prices of its competitors áfter it has produced and fixed the price for its products.

${ }^{14}$ We use $\lambda_{i}$ instead of $\lambda_{i}(\mathbf{p}, \mathbf{q})$, since $\mathbf{p}$ and $\mathbf{q}$ follow directly from the context.

${ }^{15}$ Assumption 4.2 .1 is primarily made for ease of analysis. The findings in this chapter are robust against alternative allocation rules as long as the game is symmetric and a firm's share of demand depends positively on its own level of production.
} 
Theorem 4.3.1. Assume production in advance.

(i) There exists no pure-strategy Nash equilibrium.

(ii) There exists a symmetric mixed-strategy Nash equilibrium.

Unfortunately, in the current setting it is difficult if not impossible to determine this mixed-strategy equilibrium explicitly. However, we are able to prove that (expected) equilibrium profits are zero. Below, we provide a generalized version of the analysis presented in Tasnádi (2004), who obtains a similar result for a duopoly.

For all the analyses in this section it can be easily verified that firms will never find it optimal to price below $c$ or above $p^{m}$ and supply strictly positive quantity. We therefore focus on the case where suppliers choose their actions simultaneously from $S=\left[c, p^{m}\right] \times[0, b]$. In the following, let the space of all (Borel) probability measures on $S$ be given by $P(S)$ and let $\mu_{i} \in P(S)$ denote a mixed strategy of firm $i \in N$. With $\boldsymbol{\mu}_{-i}$ we denote the mixed strategies of all firms other then firm $i$, i.e., $\boldsymbol{\mu}_{-\boldsymbol{i}}=\left(\mu_{1}, \ldots, \mu_{i-1}, \mu_{i+1}, \ldots, \mu_{n}\right)$ and $\boldsymbol{\mu}=\left(\mu_{i}, \boldsymbol{\mu}_{-\boldsymbol{i}}\right)$. The profit of firm $i$, when $\boldsymbol{\mu}$ is the joint strategy, is represented by $\pi_{i}(\boldsymbol{\mu})$. A mixed-strategy equilibrium $\boldsymbol{\mu}^{*}$ is then defined by the following condition:

$$
\pi_{i}\left(\mu_{i}, \boldsymbol{\mu}_{-i}^{*}\right) \leq \pi_{i}\left(\mu_{i}^{*}, \boldsymbol{\mu}_{-i}^{*}\right) \text { for all } i \in N \text { and } \mu_{i} \in P(S) .
$$

As we consider a symmetric situation and search for a symmetric equilibrium, we indicate a mixed-strategy Nash equilibrium and corresponding equilibrium profits of a single firm respectively by $\mu$ and $\pi^{*}$ for ease of notation. The support of $\mu$ is denoted by $\operatorname{supp}(\mu)$ and, for any price $p \in\left[c, p^{m}\right]$, $s(p) \subseteq[0, b]$ is the set of quantities $q \in[0, b]$ for which $(p, q) \in \operatorname{supp}(\mu)$. Moreover, let $\mu_{p}$ be the projection of probability measure $\mu$ to the set of prices, i.e., $\mu_{p}(B)=\mu(B \times[0, b])$ for any Borel set $B \subseteq\left[c, p^{m}\right]$. Finally, let $\sup \left\{p \in\left[c, p^{m}\right] \mid\right.$ $\left.\mu_{p}\left(\left[p, p^{m}\right]\right)=1\right\}$ and $\inf \left\{p \in\left[c, p^{m}\right] \mid \mu_{p}([c, p])=1\right\}$ be respectively denoted by $\check{p}$ and $\hat{p}$.

Clearly, if $\check{p}=c$, then $\pi^{*}=0$. In the next two lemmas, we consider the case where $\check{p}>c$.

Lemma 4.3.2. If $\check{p}>c$, then $s(\check{p})=\{D(\check{p})\}$ and $\mu_{p}(\{\check{p}\})=0$.

That is, a firm that sets $\check{p}>c$ optimally produces to meet demand at this price. The next lemma shows that producing to serve the entire market may also be optimal for prices that are above $\check{p}$.

Lemma 4.3.3. If $\check{p}>c$, then there exists a price $p^{\prime} \in\left(\check{p}, p^{m}\right]$ such that, for all $p \in\left[\check{p}, p^{\prime}\right], s(p)=\{D(p)\}$ and $\mu_{p}(\{p\})=0$. 
Finally, we use this result to establish that the infimum of all prices in the support cannot be strictly above $c$. As a result, all firms make zero expected profits in equilibrium.

Theorem 4.3.4. Assume production in advance. Then, $\check{p}=c$ and $\pi^{*}=0$.

\subsection{Cartel Problem}

In this section, we analyze the incentives of firms to establish a particular cartel contract. To do so, the game described in Section 2 is repeated an infinite number of times. In every period $t \in \mathbb{N}$, firms simultaneously make price and production decisions so as to maximize the expected discounted sum of their profit stream, where $\delta \in(0,1)$ is the common discount factor. In any period $t$, the price and quantity choices of all firms up to $t-1$ are common knowledge.

We assume that holding inventories is sufficiently costly so that firms do not find it in their interest to store unsold products.

Assumption 4.4.1. Firms do not build inventories.

Supply may exceed demand in equilibrium when production is in advance of sale. Therefore, this assumption clearly comes with a price in terms of generality. However, it is required to keep the analysis tractable. Consequently, our findings related to collusion with production in advance primarily apply to industries selling perishable or fashionable goods.

In the previous section, we found an equilibrium in the stationary setting. If we talk about competition in the infinitely repeated stage game, we refer to a situation in which all firms use strategies in which they do not use past-play or timing of current play to decide upon their current actions. Therefore, the only subgame perfect equilibrium in competition is the equilibrium in which the (symmetric) stationary outcome prevails every period. To prevent that from happening, firms can form a collusive agreement. In forming a collusive agreement, firms can choose between two types of cartels: a price cartel and a price-quota cartel. In a price cartel, the strategies of all firms take into account past choices of price only, i.e. previous quantity choices of competitors are ignored. Therefore, deviations in the past in price can be punished, but quantity can be freely chosen every moment in time without further consequences. If the firms form a price-quota cartel, in the strategies of all firms, both history of price and quantity choices matters, and any deviations of the collusive agreement will be responded to accordingly. 
For both cases, we consider an all-inclusive cartel in which all suppliers agree on a common cartel price $p^{c} \in\left(c, p^{m}\right]$. The key difference between the two types of contracts is that with a price cartel firms are free to choose their level of production, whereas a price-quota cartel additionally specifies the level and allocation of outputs. The agreed upon quota in a price-quota cartel for firm $i$ is expressed by $q_{i}^{c}$. We require collusive arrangements to be a subgame perfect equilibrium outcome of the game and it is assumed that firms adopt grim-trigger strategies to sustain collusion. ${ }^{16}$

This results in the following formal definitions of a price cartel strategy and a price-quota cartel strategy. Define $q_{i}(t)$ as the quantity that firm $i \in N$ chooses in period $t$. Let

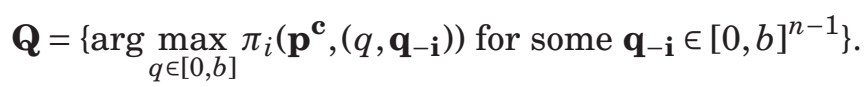

A strategy profile is a price cartel if there is a price $p^{c} \in[0, a]$ and for each firm $i$ a sequence $q_{i}(1), q_{i}(2), \ldots$ with $q_{i}(t) \in \mathbf{Q}$ for all $t$, such that each firm $i$ plays as follows:

- At period 1: set price $p^{c}$ and quantity $q_{i}(1)$;

- At each period $T>1$ : set price $p^{c}$ and quantity $q_{i}(T)$ if all firms have set prices equal to $p^{c}$ at all $t<T$, and play according to the static equilibrium strategy $\mu_{i}^{*}$ otherwise.

A strategy profile is a price-quota cartel if there is a price $p^{c} \in[0, a]$ and for each firm $i$ an individual quantity $q_{i}^{c} \in[0, b]$, such that each firm $i$ plays as follows:

- At period 1: set price $p^{c}$ and quantity $q_{i}^{c}$;

- At each period $T>1$ : set price $p^{c}$ and quantity $q_{i}^{c}$ if all firms $j \in N$ have set prices equal to $p^{c}$ and quantities equal to their individual quantity $q_{j}^{c}$ at all $t<T$, and play according to the static equilibrium strategy $\mu_{i}^{*}$ otherwise.

To begin, let us focus on a price cartel. If, given a certain collusive price, the firms always choose the same quantity level, we call this a price-cartel

\footnotetext{
${ }^{16}$ Notice that this is the most severe punishment strategy in our setting, i.e. profits in competition are zero. Therefore, if collusion is not sustainable by adopting this strategy, then collusion will not occur in any subgame perfect equilibrium.
} 
with a constant output configuration. If this is the case, the collusive value for firm $i$ is recursively defined by

$$
V_{i}\left(p^{c}, \mathbf{q}\right)=p^{c} D_{i}\left(p^{c}, \mathbf{q}\right)-c q_{i}+\delta V_{i}\left(p^{c}, \mathbf{q}\right) .
$$

Rearranging gives,

$$
V_{i}\left(p^{c}, \mathbf{q}\right)=\frac{p^{c} D_{i}\left(p^{c}, \mathbf{q}\right)-c q_{i}}{1-\delta} .
$$

As customers buy first from the cheapest supplier, maximum profit that can be earned by defecting from the cartel agreement is obtained by undercutting the cartel price slightly and producing to meet market demand at that price. Notice that this holds for all members of a price- or a price-quota cartel. Consequently, given a particular cartel price $p^{c}$, the optimal defection profit always amounts to $\left(p^{c}-c\right) D\left(p^{c}\right)$. Whether all price-cartel really have constant output configurations will be analyzed in the next sections.

As shown in the previous section, competitive behavior yields zero (expected) profits. The incentive compatibility constraint of firm $i$ is therefore given by

$$
\frac{p^{c} D_{i}\left(p^{c}, \mathbf{q}\right)-c q_{i}}{1-\delta} \geq\left(p^{c}-c\right) D\left(p^{c}\right)
$$

or

$$
\delta \geq \delta_{i}^{*}=1-\frac{p^{c} D_{i}\left(p^{c}, \mathbf{q}\right)-c q_{i}}{\left(p^{c}-c\right) D\left(p^{c}\right)}, \text { for all } i \in N .
$$

As is well-known, there may exist a plethora of sustainable cartel contracts. Yet, as we require collusion to be subgame perfect and $p^{c}>c$, all these collusive arrangements have in common that total cartel supply will not fall short of market demand. If it did, then there would be at least one firm that would benefit from increasing its production. As a result, firm demand is given by $D_{i}\left(p^{c}, \mathbf{q}\right)=\frac{q_{i}}{\sum_{j \in \Omega\left(p^{c}\right) q_{j}}} D\left(p^{c}\right)$. In turn, this implies that all firms agree to set a cartel price that maximizes total cartel value.

A price cartel thus faces the following constraint optimization problem:

$$
\max _{p} V(p, \mathbf{q})=\max _{p} \frac{p D(p)-c \sum_{j \in N} q_{j}}{1-\delta},
$$

subject to

$$
\begin{aligned}
p D_{i}(p, \mathbf{q})-c q_{i}-(1-\delta)(p-c) D(p) & \geq 0, \text { for all } i \in N, \\
\sum_{j \in N} q_{j} & \geq D(p) .
\end{aligned}
$$


The key question is then if and how firms can benefit from an additional quota agreement. In the following, we analyze this issue in more detail for markets that are characterized by production in advance.

\subsection{Collusion with Production in Advance}

\subsubsection{Price Cartel}

Suppose that the cartel prices at $p^{c} \in\left(c, p^{m}\right]$ and that production is in advance of sale. In the following, we start by considering a price cartel and then analyze if and under which conditions firms would prefer to establish a price-quota cartel instead. As a price cartel does not include an agreement on sales, all members are unrestricted in their choice of output. That is to say, every firm can individually decide on its production level without having to fear retaliation from its rivals. Consequently, a cartel member produces to maximize current profit given the output choices of its fellow members. The production level that maximizes firm i's current profit, given the output choices of its fellow members, we will call the best reply production choice $q_{i}^{*}\left(p^{c}, \mathbf{q}-\mathbf{i}\right)$.

The next result specifies the optimal production decision at a given cartel price.

Lemma 4.5.1. Assume production in advance and a price cartel with common cartel price $p^{c} \in\left(c, p^{m}\right]$. For all $i \in N$, the best reply production choice $q_{i}^{*}\left(p^{c}, \mathbf{q}_{-\mathbf{i}}\right)$ is given by:

$q_{i}^{*}\left(p^{c}, \mathbf{q}_{-\mathbf{i}}\right)= \begin{cases}D\left(p^{c}\right)-\sum_{j \in N \backslash\{i\}} q_{j} & \text { if } \sum_{j \in N \backslash\{i\}} q_{j} \leq D\left(p^{c}\right) c / p^{c} \\ \sqrt{\frac{p^{c} D\left(p^{c}\right)\left(\sum_{j \in N \backslash i\}} q_{j}\right)}{c}}-\sum_{j \in N \backslash\{i\}} q_{j} & \text { if } D\left(p^{c}\right) c / p^{c}<\sum_{j \in N \backslash\{i\}} q_{j} \leq D\left(p^{c}\right) p^{c} / c \\ 0 & \text { if } \sum_{j \in N \backslash\{i\}} q_{j}>D\left(p^{c}\right) p^{c} / c .\end{cases}$

Observe that the first in combination with the second or the third optimal response cannot occur in equilibrium. Likewise, we can exclude the possibility that in equilibrium some firms adopt the middle best response and some firms produce nothing. To see this, notice that in this case it must hold that $\sum_{i \in N} q_{i}=\sqrt{\frac{p^{c} D\left(p^{c}\right)\left(\sum_{j \in N \backslash i\}} q_{j}\right)}{c}}$ and $\sum_{j \in N \backslash\{i\}} q_{j} \leq D\left(p^{c}\right) p^{c} / c$. This implies $\sum_{i \in N} q_{i} \leq \sqrt{\frac{p^{c} D\left(p^{c}\right)\left(D\left(p^{c}\right) p^{c} / c\right)}{c}}=D\left(p^{c}\right) p^{c} / c$. If a firm would produce nothing, then $\sum_{j \in N \backslash\{i\}} q_{j} \leq D\left(p^{c}\right) p^{c} / c$. Yet, zero production is only a best response when $\sum_{j \in N \backslash\{i\}} q_{j}>D\left(p^{c}\right) p^{c} / c$; a contradiction. This leaves two possibilities that can occur in equilibrium. Either, all firms produce according to the first 
best response (i.e., $\sum_{i \in N} q_{i}=D\left(p^{c}\right)$ ), or all firms set their outputs such that $\sum_{i \in N} q_{i}=\sqrt{\frac{p^{c} D\left(p^{c}\right)\left(\sum_{j \in N \backslash i i} q_{j}\right)}{c}}$. In the latter case, total supply exceeds market demand.

The next result shows that, in the event of overproduction, all firms produce the same quantity.

Lemma 4.5.2. If $q_{v}^{*}\left(p^{c}, \mathbf{q}_{-\mathbf{v}}\right)=\sqrt{\frac{p^{c} D\left(p^{c}\right)\left(\sum_{j \in N \backslash v\}} q_{j}\right)}{c}}-\sum_{j \in N \backslash\{v\}} q_{j}$ and $q_{w}^{*}\left(p^{c}, \mathbf{q}_{-\mathbf{w}}\right)=$ $\sqrt{\frac{p^{c} D\left(p^{c}\right)\left(\sum_{j \in N \backslash\{w\}} q_{j}\right)}{c}}-\sum_{j \in N \backslash\{w\}} q_{j}$, then $q_{v}^{*}\left(p^{c}, \mathbf{q}_{-\mathbf{v}}\right)=q_{w}^{*}\left(p^{c}, \mathbf{q}_{-\mathbf{w}}\right)$ for all $v, w \in$ $N$.

The previous two findings are useful in characterizing the set of subgame perfect equilibria. It appears that equilibrium production decisions in part depend on the level of the cartel price.

Theorem 4.5.3. Assume production in advance. If a price cartel $\left(p^{c}, \mathbf{q}^{*}\right)$ is a subgame perfect Nash equilibrium, then for all $i \in N$,

(i) $p^{c} \leq \frac{c n}{n-1}, q_{i}^{*}\left(p^{c}, \mathbf{q}_{-\mathbf{i}}\right)=D\left(p^{c}\right)-\sum_{j \in N \backslash\{i\}} q_{j}^{*}\left(p^{c}, \mathbf{q}_{-\mathbf{j}}\right)$ and $q_{i}^{*}\left(p^{c}, \mathbf{q}_{-\mathbf{i}}\right) \geq\left(\frac{p^{c}-c}{p^{c}}\right) D\left(p^{c}\right)$,

(ii) $p^{c}>\frac{c n}{n-1}$ and $q_{i}^{*}\left(p^{c}, \mathbf{q}_{-\mathbf{i}}\right)=\frac{(n-1) p^{c} D\left(p^{c}\right)}{c n^{2}}$.

Thus, in equilibrium, the collusive value for firm $i$ is given by

$$
V_{i}\left(p^{c}, \mathbf{q}^{*}\right)= \begin{cases}\frac{\left(p^{c}-c\right)\left(D\left(p^{c}\right)-\sum_{j \in N \backslash i\}} q_{j}^{*}\left(p^{c}, \mathbf{q}_{-\mathbf{j}}\right)\right)}{1-\delta} & \text { for } p^{c} \leq \frac{c n}{n-1} \text { and } q_{i}^{*}\left(p^{c}, \mathbf{q}_{-\mathbf{i}}\right) \geq \\ & \left(\frac{p^{c}-c}{p^{c}}\right) D\left(p^{c}\right) \text { for all } i \in N, \text { or } \\ \frac{p^{c} D\left(p^{c}\right)}{(1-\delta) n^{2}} & \text { for } p^{c}>\frac{c n}{n-1} \text { and } q_{i}^{*}\left(p^{c}, \mathbf{q}_{-\mathbf{i}}\right)= \\ & \frac{(n-1) p^{c} D\left(p^{c}\right)}{c n^{2}} \text { for all } i \in N .\end{cases}
$$

The next issue is to find the price that maximizes the total cartel value. Following Theorem 4.5.3, we can distinguish two cases. First, if the cartel sets a price $p \leq \frac{c n}{n-1}$, then $q_{i}^{*}\left(p, \mathbf{q}_{-\mathbf{i}}\right)=D(p)-\sum_{j \in N \backslash\{i\}} q_{j}^{*}\left(p, \mathbf{q}_{-\mathbf{j}}\right)$ for all $i \in N$. Therefore, the incentive compatibility constraint as given by (4.5) reduces to

$$
\delta \geq \hat{\delta}_{i}^{*}=1-\frac{q_{i}^{*}\left(p, \mathbf{q}_{-\mathbf{i}}\right)}{\sum_{j \in N} q_{j}^{*}\left(p, \mathbf{q}_{-\mathbf{j}}\right)} \text {, for all } i \in N
$$

which does not directly depend on the cartel price. As a result, if $p^{m} \leq$ $\frac{c n}{n-1}$, then the cartel optimally sets the monopoly price. Note that, since $\max _{i \in N} \hat{\delta}_{i}^{*} \geq 1-\frac{1}{n}$, this can only occur if $\delta \geq 1-\frac{1}{n}$.

Second, all cartel participants optimally produce $q^{*}(p)=\frac{(n-1) p D(p)}{c n^{2}}$ at 
any price $p>\frac{c n}{n-1}$. In this case, the incentive compatibility constraint as given by (4.5) is therefore given by

$$
\delta \geq \tilde{\delta^{*}}(p)=1-\frac{p}{n^{2}(p-c)},
$$

which is the same for all firms and directly depends on price. In fact, $\tilde{\delta}^{*}(p)$ is an increasing and concave function of $p$ and, since $\tilde{\delta}^{*}\left(\frac{c n}{n-1}\right)=1-\frac{1}{n}$, also this incentive compatibility constraint can only hold as long as $\delta \geq 1-\frac{1}{n}$.

Given that the cartel price exceeds $\frac{c n}{n-1}$, define $p^{*}$ as the unconstrained solution of the cartel problem:

$$
\frac{d V\left(p, \mathbf{q}^{*}(p)\right)}{d p}=\frac{p D^{\prime}(p)+D(p)}{(1-\delta) n^{2}}=0 .
$$

Thus, $p^{*}$ solves $p^{*} D^{\prime}\left(p^{*}\right)+D\left(p^{*}\right)=0$ and therefore $p^{m}>p^{*}$. Additionally, define $\tilde{p}$ as the constrained solution, i.e., $\delta=1-\frac{\tilde{p}}{n^{2}(\tilde{p}-c)}$.

Using the foregoing analysis, the next Proposition summarizes the optimal pricing decision of a price-fixing cartel.

Proposition 4.5.4. Assume production in advance and $\delta \geq 1-\frac{1}{n}$. The optimal cartel price $p^{c}$ is specified as follows:

1. Suppose $p^{m} \leq \frac{c n}{n-1}$.

(a) If $\delta \geq \hat{\delta_{i}^{*}}$ for all $i \in N$, then $p^{c}=p^{m}$.

2. Suppose $p^{m}>\frac{c n}{n-1}$.

(a) If $p^{*}<\frac{c n}{n-1}$, then $p^{c}=\frac{c n}{n-1}$.

(b) If $\delta<\tilde{\delta}^{*}\left(p^{*}\right)$ and $p^{*} \geq \frac{c n}{n-1}$, then $p^{c}=\tilde{p}$.

(c) If $\delta \geq \tilde{\delta^{*}}\left(p^{*}\right)$ and $p^{*} \geq \frac{c n}{n-1}$, then $p^{c}=p^{*}$.

\subsubsection{Price-Quota Cartel}

Above, we have characterized the optimal collusive strategy of a price-fixing cartel. The remaining issue is to analyze if and how suppliers could benefit from an additional quota agreement. In the following, we show that there are at least three arguments in favor of a price-quota cartel when production is in advance of sales.

First, if $p^{c} \leq \frac{c n}{n-1}$, then by Theorem 4.5.3 production levels must be such 
that $q_{i}^{*}\left(p^{c}, \mathbf{q}_{-\mathbf{i}}\right)=D\left(p^{c}\right)-\sum_{j \in N \backslash\{i\}} q_{j}^{*}$ and $q_{i}^{*}\left(p^{c}, \mathbf{q}_{-\mathbf{i}}\right) \geq\left(\frac{p^{c}-c}{p^{c}}\right) D\left(p^{c}\right)$. Thus, the market should clear while ensuring that every cartel participant has a sufficiently high level of sales. Combining both these requirements yields the following rather striking result.

Corollary 4.5.5. Assume production in advance and $p^{c} \leq \frac{c n}{n-1}$. The market share of every cartel member must (weakly) exceed the Lerner index for a price cartel to be stable. ${ }^{17}$

A price cartel therefore faces a coordination problem when it prices at $p^{c}<\frac{c n}{n-1} .{ }^{18}$ This is the case when $p^{m}<\frac{c n}{n-1}$. As in this case there seems to be no natural division of outputs, an explicit arrangement on market shares may be required to solve this coordination problem.

In addition, a quota agreement may also be needed to solve the incentive problem when $p^{m} \leq \frac{c n}{n-1}$. Observe that the minimum critical discount factor is always weakly larger than $1-\frac{1}{n}$, which is obtained with an equal division of sales. We therefore conclude that there exists no viable cartel when $\delta<$ $1-\frac{1}{n}$. Yet, given that $\delta \geq 1-\frac{1}{n}$, viability of a cartel may still require a market sharing scheme to prevent the smallest member(s) from leaving the ring.

Theorem 4.5.6. Assume production in advance, $\delta \geq 1-\frac{1}{n}$ and $p^{m} \leq \frac{c n}{n-1}$.

(i) If $1-\frac{1}{n} \leq \delta<1-\frac{q_{i}^{*}\left(p^{c}, \mathbf{q}_{-\mathbf{i}}\right)}{\sum_{j \in N} q_{j}^{*}\left(p^{c}, \mathbf{q}_{-\mathbf{j}}\right)}$ for some firm $i \in N$, then there exists only a viable price-quota cartel, not a viable price cartel, and

(ii) If $1-\frac{q_{i}^{*}\left(p^{c}, \mathbf{q}_{-\mathbf{i}}\right)}{\sum_{j \in N} q_{j}^{*}\left(p^{c}, \mathbf{q}_{-\mathbf{j}}\right)} \leq \delta$ for all $i \in N$, then there exists both a viable price cartel and a price-quota cartel.

Finally, if $p^{m}>\frac{c n}{n-1}$, then Proposition 4.5.4 reveals that a price-fixing cartel is feasible. Moreover, in this case firms have no coordination problem as they optimally produce the same amount of products at any cartel price $p^{c} \geq \frac{c n}{n-1}$. Here, the only reason for suppliers to establish a price-quota cartel is that it generates more profits than a price cartel. In particular, we know by Proposition 4.5.4 that the profit-maximizing price of a price cartel is below the monopoly price. Implementing an optimal quota scheme allows firms to avoid excessive production and to sustain the monopoly price, thereby increasing the collusive value.

\footnotetext{
${ }^{17}$ The Lerner index describes the relative profit margin, i.e. $\frac{p-c}{p}$, of a firm.

${ }^{18}$ This coordination problem is absent for $p^{c}=\frac{c n}{n-1}$ as then all members find it optimal to produce the same amount of products.
} 
Theorem 4.5.7. Assume production in advance, $\delta \geq 1-\frac{1}{n}$ and $p^{m}>\frac{c n}{n-1}$. There exists a price-quota cartel for which $V_{i}\left(p^{c}, \mathbf{q}^{\mathbf{c}}\right)>V_{i}\left(p^{c}, \mathbf{q}^{*}\left(p^{c}\right)\right)$ for all $i \in N$. An optimal price-quota cartel contract has all firms pricing at $p^{m}$ and a total production of $D\left(p^{m}\right)$.

In conclusion, if market demand is sufficiently elastic $\left(p^{m} \leq \frac{c n}{n-1}\right)$, then a quota agreement may be required to solve a coordination and an incentive problem. If a price-quota cartel is necessary to overcome incentive problems, all else unchanged, the cartel leads to a convergence of market shares. By contrast, when market demand is sufficiently inelastic $\left(p^{m}>\frac{c n}{n-1}\right)$, the sole reason for establishing an additional quota agreement is that it allows the cartel to sustain a higher price while reducing costs due to overproduction. As $\frac{c n}{n-1}$ is decreasing in $n$, the latter situation is more likely the larger the number of firms in the industry. Contrary to conventional wisdom, this implies that the coordination problem may be more pronounced the fewer the number of cartel participants, all else equal.

\subsection{Production to Order}

In this chapter, next to production in advance, we consider another mode of production: production to order. Many of the assumptions made for production in advance are the same as those for production to order. Therefore, in this section, we will only explicitly define the assumptions that differ from those in the previous sections.

The fundamental difference between both settings lies in the way in which a firms' demand is determined. When firms produce to order, each firm only sets its price, not its production level. The consumers first buy from a supplier charging the lowest price. In case the cheapest supplier(s) choose(s) not to produce up to demand, consumers go to have their residual demand fulfilled at the second cheapest supplier(s), etcetera. We will assume that, as long as there are no agreements made between firms about allocation of demand, each firm always supplies the demand it faces.

Since realization of demand is known before production takes place, the allocation of customers when two or more firms charge the same price does not depend on firms' actual production levels. In this case, we use $\alpha$ to describe a firm's share of market demand. Particularly, if all suppliers charge the same price and total supply does not fall short of market demand at that price, then $\alpha_{i}$ is the (exogenously given) market share of firm $i$. 
Firm $i$ 's demand, when there are no agreements made between firms about allocation of demand, is therefore of the following structure:

$$
D_{i}\left(p_{i}, \mathbf{p}_{-\mathbf{i}}\right)= \begin{cases}\frac{\alpha_{i}}{\sum_{j \in \Omega\left(p_{i}\right)} \alpha_{j}} & \text { if } p_{i} \leq p_{j} \text { for all } j \in N \backslash\{i\} \\ 0 & \text { otherwise }\end{cases}
$$

Consequently, the profit of firm $i$ is given by

$$
\pi_{i}\left(p_{i}, \mathbf{p}_{-\mathbf{i}}\right)=\left(p_{i}-c\right) D_{i} .
$$

\subsubsection{Static Nash Equilibrium}

Firms in this setting basically compete in price. Therefore, the static Nash Equilibrium here is the same as in the Bertrand Paradox. That is, there exists a pure-strategy Nash equilibrium with all firms (or at least two) pricing at $c$ and producing to meet demand. There is no other Nash equilibrium in which one or more suppliers make a positive profit. Consequently, it can be concluded that stationary profits are zero. This provides a strong incentive for firms to engage in a cartel. But what (type of) cartel contract is most likely to be formed? It is this issue that we address in the next section.

\subsubsection{Collusion}

If the firms form a price cartel, then firm $i$ faces a demand of $\alpha_{i} D\left(p^{c}\right)$. The collusive value of this price cartel for firm $i$ is recursively defined by

$$
V_{i}\left(p^{c}\right)=\left(p^{c}-c\right) \alpha_{i} D\left(p^{c}\right)+\delta V_{i}\left(p^{c}\right),
$$

which can be rewritten as

$$
V_{i}\left(p^{c}\right)=\frac{\left(p^{c}-c\right) \alpha_{i} D\left(p^{c}\right)}{1-\delta} .
$$

The incentive compatibility constraint of firm $i$ is therefore given by

$$
\delta \geq \delta_{i}^{*}=1-\alpha_{i}, \text { for all } i \in N .
$$

As before, sustainability of collusion requires $\delta \geq 1-\frac{1}{n}$, which is obtained with an equal division of sales. Yet, unlike with production in advance, a price cartel will not face a coordination problem. Also, the incentive constraint is independent of the elasticity of market demand. In fact, given 
that $\delta \geq 1-\frac{1}{n}$, whether or not price collusion is viable solely depends on the size distribution of cartel participants. Specifically, feasibility of collusion requires the market share of the smallest cartel member(s) to be sufficiently large. As the critical discount factor is independent of the cartel price, the cartel will optimally set the monopoly price, i.e., $p^{c}=p^{m}$. The next result therefore closely resembles Theorem 4.5.6 above.

Theorem 4.6.1. Assume production to order and $\delta \geq 1-\frac{1}{n}$.

(i) If $1-\frac{1}{n} \leq \delta<1-\frac{\alpha_{i}}{\sum_{j \in N} \alpha_{j}}$ for some firm $i \in N$, then there exists only a viable price-quota cartel, not a viable price cartel, and

(ii) If $\delta \geq 1-\frac{\alpha_{i}}{\sum_{j \in N} \alpha_{j}}$ for all $i \in N$, then there exists both a viable price cartel and a price-quota cartel.

When firms produce to order, a price cartel and a price-quota cartel are equally profitable as in both cases the cartel mimics a multi-plant monopolist. Consequently, the only reason for firms to adopt a market sharing scheme is that without such an arrangement collusion may not be sustainable. In particular, the cartel may have to agree on a redistribution of sales from the larger to the smaller members. ${ }^{19}$ There are several ways in which firms can arrange a more equal division of sales. For example, larger firms may simply refuse to serve part of their demand so as to increase the residual demand for the products of smaller members. Alternatively, the cartel may adopt a more sophisticated system of end-of-the-year buy backs to ensure that every member meets its pre-arranged output level. It is noteworthy that such agreements have been observed several times in antitrust practice. ${ }^{20}$

\subsection{Discussion}

The above analysis has been conducted under several assumptions, some of which we believe warrant some discussion. In the following, we relate our work to literature that considers settings with cost heterogeneity, demand uncertainty and private information.

By studying collusion in a price-quantity oligopoly, our analysis sheds some

\footnotetext{
${ }^{19}$ In this chapter, we derive results for a given cartel size. Alternatively, when cartel formation is assumed endogenous, it may be more optimal for the larger firms to form a less than all-inclusive cartel leaving out the smaller firms. This possibility is analyzed in Bos and Harrington (2010).

${ }^{20}$ See, for example, Harrington (2006).
} 
light on what type of cartel we may expect in particular type of industries. Our findings suggest that we often may expect firms to agree on both prices and quantities, albeit for various reasons. We have derived results in a setting where both prices and quantities are public information and firms have accurate knowledge about cost and demand functions. Clearly, relaxing one or more of these assumptions may provide alternative explanations for when to expect firms to establish a price-quota cartel. Existing literature has offered various rationales for full collusion in the presence of cost and demand shocks and in case strategic choice variables are private information. Here, we briefly discuss this related literature.

Let us first discuss the possibility of cost heterogeneity. In our setting, differences in firm size are unrelated to differences in unit costs. Consequently, a market-sharing scheme when implemented is also not driven by efficiency considerations. This is unlikely to hold in general as differences in unit costs may give rise to asymmetric incentive schemes. For instance, one may conjecture a cartel to allocate a relatively large market share to more efficient members as these have more to gain from defection and less to fear from retaliation. ${ }^{21}$ In this respect, Harrington (1991) shows that whether or not an optimal market sharing rule is sensitive to cost differences in part depends on the level of the discount factor. Specifically, the market sharing rule is independent of firms' unit costs when the discount factor is relatively low, but not when firms are sufficiently patient.

The potential impact of cost heterogeneity on collusion has also been analyzed in a setting of fluctuating demand conditions. Choi et al. (1985), for example, consider a static framework in which firms negotiate price and market shares to establish an efficient cartel agreement. Assuming cost heterogeneity and demand uncertainty, the prediction is that the cartel arrangement includes averaging of unit production costs. Moreover, this study predicts a convergence of market shares when market demand declines. The reason being that the small firms (i.e., high cost firms) must earn sufficient profits for the cartel to remain effective. Alternatively, firms may engage in involuntary periodic price wars to sustain collusion. Indeed, as Green and Porter (1984) predicts, a temporary breakdown in prices may be required to maintain a collusive scheme when demand conditions are fluctuating and firms' quantities are private information.

Market share schemes potentially also play an important role when pro-

\footnotetext{
${ }^{21}$ Note that sustainability of collusion with differences in unit costs may also require a more equal division of sales, because efficient firms have more to gain from a cartel.
} 
duction costs are not publicly known. As Athey and Bagwell (2001) points out, market share agreements may allow producers to collude perfectly when they experience privately observed cost shocks in each period. By exchanging future market share favors efficiently, first-best profits can be attained when firms are sufficiently patient. Such a rather sophisticated price-quota cartel is preferred as it induces a high cost firm to reveal its identity, thereby allowing the cartel to produce efficiently. In a comparable setting, Athey et al. (2004) shows that optimal collusion may induce firms to fix both prices and market shares. In particular, this implies that the cartel forgoes productive efficiency as low cost firms produce relatively too little and high costs firms produce relatively too much. Yet, it is also found that when firms are sufficiently impatient, effectiveness of collusion may require low cost firms to set a lower price in order to gain more market share.

A couple of recent studies has considered collusion in a setting where prices or quantities are private information. Harrington and Skrzypacz (2007) shows that when prices are not publicly observable (but firms' quantities are), then the mere threat of (symmetric) price wars may be insufficient to sustain collusion. Yet, collusion may be sustainable through an asymmetric punishment scheme that prescribes firms that sold in excess of their quota to compensate those members that sold under quota. In a similar fashion, Harrington and Skrzypacz (2010) shows that firms with high sales may have an incentive to compensate members with low sales to sustain collusion in an environment where both prices and quantities are private information. That firms do not need much information to collude effectively has been recently confirmed by Hörner and Jamison (2007). This study finds that firms require hardly any information to collude almost perfectly. However, to obtain this result, firms need to agree on both prices and market shares.

The studies mentioned above reveal that there are distinct possibilities for price-fixing firms to benefit from a market sharing scheme. In this respect, our findings are complementary and suggest that price-quota cartels are likely to be the rule. Put differently, even in a world where cost and demand are known and stable and actions are publicly observable, firms often benefit from a market sharing agreement. 
4.8. Concluding Remarks

\subsection{Concluding Remarks}

Why do we observe various types of cartel contracts in antitrust practice? In this study, we have sought to shed some light on one specific sort of cartel heterogeneity: price versus price-quota cartels. Existing literature has shown that an additional agreement on production levels may be needed to overcome incentive problems that arise due to imperfect information and demand uncertainty. This chapter provides an alternative explanation. In the context of an infinitely repeated game with complete information, sustainability of collusion may still require an agreement on sales levels. Specifically, the market share of the smallest cartel member must be sufficiently large, which may induce firms to arrange a more equal division of outputs. This holds when firms produce to order and when production is in advance of sale and market demand is sufficiently elastic. As to the latter, an arrangement on outputs may additionally help firms to solve a coordination problem. Moreover, with production in advance, establishing an output ceiling is always profitable when market demand is sufficiently inelastic. Consequently, there are several rationales for the existence of both price and price-quota cartels, even in a world of certainty and perfect information.

These results have potentially important implications in light of antitrust enforcement. For instance, industries with price increases in conjunction with a decline in sales volume and converging market shares should, ceteris paribus, be considered suspect of anti-competitive practices. Also, refusals to deal at relatively high prices in combination with relatively low capacity utilization may indicate the presence of a cartel. However, such observations should always be judged while taking account of the idiosyncrasies of a particular industry. Indeed, our analysis suggests that market characteristics may play a vital role in the design of a cartel contract. In particular, knowing when and where to expect a price-quota cartel is likely to increase the effectiveness of antitrust enforcement as the chance of discovering physical evidence is higher, all else equal. The reason being that, unlike with price collusion, it is difficult to see how firms can coordinate their sales levels without communicating explicitly. Yet, to what extent our theoretical findings are helpful in detecting cartels is ultimately an empirical question. We leave this issue for future research. 


\section{A Appendix}

\section{Proof of Theorem 4.3.1.}

(i) If all firms price above $c$, then there is at least one firm that finds it optimal to undercut the lowest price slightly and serve market demand at that price. Therefore, let $M=\left\{i \in N \mid p_{i}=c\right\}$ the set of firms that price at $c$, where $|M| \geq 1$. In this case, for all $i \in M, q_{i}>D_{i}\left(c, q_{i}, \mathbf{p}_{-\mathbf{i}}, \mathbf{q}_{-\mathbf{i}}\right)$ gives $\pi_{i}<0$, whereas $q_{i}=D_{i}\left(c, q_{i}, \mathbf{p}_{-\mathbf{i}}, \mathbf{q}_{-\mathbf{i}}\right)$ gives $\pi_{i}=0$. Thus, a firm pricing at $c$ will not produce in excess of its demand. This implies that there exists a firm $j \in M$ for which it holds that $D(c)-\sum_{i \in M \backslash\{j\}} q_{i}>0$. As a result, this firm $j$ can do better by charging a higher price and produce to meet its residual demand at that price. Hence, there exists no pure-strategy Nash equilibrium.

(ii) In our setting, the strategy space $A$ is compact and the game is symmetric. Therefore we can apply Corollary 5.3 of Reny (1999). According to this corollary, there exists a symmetric mixed strategy equilibrium in our game if its mixed extension is diagonally payoff secure and each $\pi_{i}(\mu, \ldots, \mu)$ is upper semicontinuous as a function of $\mu$ on $P(A)$. Here, $P(A)$ is the space of all (Borel) probability measures on $A$ endowed with the weak topology.

- Upper semicontinuity: To proof that each $\pi_{i}(\mu, \ldots, \mu)$ is upper semicontinuous as a function of $\mu$ on $P(A)$, we have to show that $\lim \sup \pi_{i}\left(\mu^{t}, \ldots, \mu^{t}\right) \leq$ $\pi_{i}(\mu, \ldots, \mu)$ whenever $\lim _{t \rightarrow \infty} \mu^{t}=\mu$. This is equivalent to showing that every converging subsequence has a limit that is smaller or equal to $\pi_{i}(\mu, \ldots, \mu)$. Therefore, assume w.l.o.g. that $\lim _{t \rightarrow \infty} \pi_{i}\left(\mu^{t}, \ldots, \mu^{t}\right)=y$ and let $x=\pi_{i}(\mu, \ldots, \mu)$. Since $\sum_{i \in N} \pi_{i}$ is continuous and all firms play the same strategy in a symmetric game, it holds that $n y=\lim _{t \rightarrow \infty} \sum_{i \in N} \pi_{i}\left(\mu^{t}, \ldots, \mu^{t}\right)=$ $\sum_{i \in N} \pi_{i}(\mu, \ldots, \mu)=n x$. Therefore, $x=y$ and $\lim _{t \rightarrow \infty} \pi_{i}\left(\mu^{t}, \ldots, \mu^{t}\right)=\pi_{i}(\mu, \ldots, \mu)$. This implies that each $\pi_{i}(\mu, \ldots, \mu)$ is not only upper semicontinuous as a function of $\mu$ on $P(A)$, but continuous as well.

- Diagonally payoff secure: To proof that our setting is diagonally payoff secure, we have to show that for every $\mu \in P(A)$ and every $\varepsilon>0$, each firm $i$ can secure a payoff $\pi_{i}(\mu, \ldots, \mu)-\varepsilon$ along the diagonal at $(\mu, \ldots, \mu)$. Firm $i$ can secure a payoff of $\pi_{i}(\mu, \ldots, \mu)-\varepsilon$ along the diagonal at $(\mu, \ldots, \mu)$ if there exists a $\bar{\mu} \in P(A)$ such that $\pi_{i}\left(\mu^{\prime}, \ldots, \bar{\mu}, \ldots, \mu^{\prime}\right) \geq \pi_{i}(\mu, \ldots, \mu)-\varepsilon$ for all $\mu^{\prime}$ in some open neighborhood of $\mu \in P(A)$.

Now, for every $\delta>0$ and every Borel subset $B$ of $A$ define

$$
B^{\delta}:=\left\{\left(p^{\prime}, q\right) \in A \mid \text { there is }(p, q) \in B \text { such that } p^{\prime}=p+\delta\right\} .
$$


For a mixed strategy $\mu$ on $A$ define the mixed strategy $\mu^{\delta}$ by

$$
\mu^{\delta}(B):=\mu\left(B^{\delta}\right)+\mu(\{(p, q) \in B \mid p<\delta\})
$$

for every Borel set $B$. Finally, let $\varepsilon>0$ and let $\mu$ be a mixed strategy on $A$. Then there is a $\delta>0$ such that $u_{i}\left(\mu^{\prime}, \ldots, \mu^{\prime}, \mu^{\delta}, \mu^{\prime}, \ldots, \mu^{\prime}\right) \geq$ $u_{i}(\mu, \ldots, \mu)-\varepsilon$ for all $\mu^{\prime}$ close enough to $\mu$. This last line follows because, given that the other firms do not change their mixed strategies too much, if firm $i$ slightly lowers its price, this at most slightly reduces its profit.

Proof of Lemma 4.3.2. We prove that $s(\check{p})=\{D(\check{p})\}$ by deriving a contradiction. In our game, the support is compact and therefore $s(\check{p}) \neq \varnothing$.

First, we will show that for all $p \in\left[\check{p}, p^{m}\right]$, for all $q \in s(p)$ it holds that $q \leq$ $D(p)$. Define $B_{\varepsilon}(p, q)$ as the open $\varepsilon$-ball centered at $(p, q)$. Assume $q>D(p)$ and let $\varepsilon>0$ be small enough, such that for all $\left(p_{i}, q_{i}\right) \in B_{\varepsilon}(p, q)$ it holds that $q_{i}>D\left(p_{i}\right)$. Let $(\tilde{p}, \tilde{q})$ be an arbitrary element in $B_{\varepsilon}(p, q)$. Then, given any $\left(\boldsymbol{p}_{-\boldsymbol{i}}, \boldsymbol{q}_{-\boldsymbol{i}}\right)$, the actions chosen by the other $n-1$ firms, firm $i$ 's profit is

$$
\pi_{i}\left(\tilde{p}, \tilde{q}, \boldsymbol{p}_{-i}, \boldsymbol{q}_{-\boldsymbol{i}}\right)=\tilde{p} \frac{\tilde{q}}{\sum_{j \in \Omega(\tilde{p})} q_{j}}\left(D(\tilde{p})-\sum_{k \in \Delta(\tilde{p})} q_{k}\right)^{+}-c \tilde{q} .
$$

Instead, if firm $i$ would choose the action $(\tilde{p}-\delta, D(\tilde{p}-\delta))$, where $\delta>0$ is chosen such that $D(\tilde{p}-\delta)<\tilde{q}$ and $\delta D(\tilde{p})<c(\tilde{q}-D(\tilde{p}-\delta))$, its profit is

$$
\pi_{i}\left(\tilde{p}-\delta, D(\tilde{p}-\delta), \boldsymbol{p}_{-\boldsymbol{i}}, \boldsymbol{q}_{-\boldsymbol{i}}\right)=(\tilde{p}-\delta) \frac{\tilde{q}}{\sum_{j \in \Omega(\tilde{p}-\delta)} q_{j}}\left(D(\tilde{p}-\delta)-\sum_{k \in \Delta(\tilde{p}-\delta)} q_{k}\right)^{+}-c D(\tilde{p}-\delta) .
$$

Note that since $D(\tilde{p}-\delta)<\tilde{q}$, it holds that if $\left(D(\tilde{p})-\sum_{k \in \Delta(\tilde{p})} q_{k}\right)^{+}=0$, then $(\tilde{p}-\delta, D(\tilde{p}-\delta))$ is a strict improvement over $(\tilde{p}, \tilde{q})$. Now, if $(D(\tilde{p})-$ $\left.\sum_{k \in \Delta(\tilde{p})} q_{k}\right)^{+}>0$, then

$$
\begin{aligned}
\pi_{i}\left(\tilde{p}-\delta, D(\tilde{p}-\delta), \boldsymbol{p}_{-\boldsymbol{i}}, \boldsymbol{q}_{-\boldsymbol{i}}\right) & >(\tilde{p}-\delta)\left(D(\tilde{p})-\sum_{k \in \Delta(\tilde{p})} q_{k}\right)-c D(\tilde{p}-\delta) \\
& >(\tilde{p}-\delta)\left(D(\tilde{p})-\sum_{k \in \Delta(\tilde{p})} q_{k}\right)+\delta D(\tilde{p})-c \tilde{q} \\
& =\tilde{p}\left(D(\tilde{p})-\sum_{k \in \Delta(\tilde{p})} q_{k}\right)-c \tilde{q}+\delta \sum_{k \in \Delta(\tilde{p})} q_{k} \\
& \geq \tilde{p}\left(D(\tilde{p})-\sum_{k \in \Delta(\tilde{p})} q_{k}\right)-c \tilde{q} \\
& \geq \tilde{p} \frac{\tilde{q}}{\sum_{j \in \Omega(\tilde{p})} q_{j}}\left(D(\tilde{p})-\sum_{k \in \Delta(\tilde{p})} q_{k}\right)-c \tilde{q} \\
& =\pi_{i}\left(\tilde{p}, \tilde{q}, \boldsymbol{p}_{-\boldsymbol{i}}, \boldsymbol{q}_{-\boldsymbol{i}}\right) .
\end{aligned}
$$


Here, the first inequality holds because of the following. First, note that $\Omega(\tilde{p}-\delta) \backslash\{i\} \cup \Delta(\tilde{p}-\delta) \subseteq \Delta(\tilde{p}), \Omega(\tilde{p}-\delta) \backslash\{i\} \cap \Delta(\tilde{p}-\delta)=\varnothing$ and $D(\tilde{p}-\delta)>D(\tilde{p})$. This gives

$$
\begin{aligned}
D(\tilde{p})-\sum_{k \in \Delta(\tilde{p})} q_{k} & <D(\tilde{p}-\delta)-\sum_{k \in \Delta(\tilde{p}-\delta)} q_{k}-\sum_{j \in \Omega(\tilde{p}-\delta) \backslash\{i\}} q_{j} \\
& \leq \frac{\tilde{q}}{\sum_{j \in \Omega(\tilde{p}-\delta)} q_{j}}\left(D(\tilde{p}-\delta)-\sum_{k \in \Delta(\tilde{p}-\delta)} q_{k}\right) .
\end{aligned}
$$

The second inequality follows from $\delta D(\tilde{p})<c(\tilde{q}-D(\tilde{p}-\delta))$. So, also when the residual demand is strictly positive, the action $(\tilde{p}-\delta, D(\tilde{p}-\delta))$ gives a strictly higher profit than $(\tilde{p}, \tilde{q})$. This implies that, for any $(\tilde{p}, \tilde{q}) \in B_{\varepsilon}(p, q)$, there exists a $\delta$ such that it holds that $(\tilde{p}-\delta, D(\tilde{p}-\delta))$ gives a strictly higher payoff and therefore $q \notin s(p)$.

Next, assume that $q \in s(\check{p})$ and $q<D(\check{p})$. The maximum profit that a firm can make in any point in an open $\varepsilon$-ball centered at $(\check{p}, q)$ is strictly smaller than $(\check{p}+\varepsilon-c)(q+\varepsilon)$. Since $(\check{p}-c) D(\check{p})>(\check{p}-c) q$, as $\varepsilon \rightarrow 0$, there always exists a $\eta>0$ such that

$$
(\check{p}-\eta-c) D(\check{p}-\eta)>(\check{p}+\varepsilon-c)(q+\varepsilon) .
$$

That is, there always exists a price-quantity combination $(\check{p}-\eta, D(\check{p}-\eta))$ that gives a strictly higher profit than any point in $B_{\varepsilon}(\check{p}, q)$. This contradicts $q \in s(\check{p})$. Thus, we conclude $s(\check{p})=\{D(\check{p})\}$.

Suppose there is an atom at price $\check{p}$. In that case, $\pi_{i}\left((\check{p}, D(\check{p})), \boldsymbol{\mu}_{-\boldsymbol{i}}\right)<(\check{p}-$ $c) D(\check{p})$. This implies that there exists a small enough $\delta>0$ such that

$$
\begin{aligned}
\pi_{i}\left((\check{p}-\delta, D(\check{p}-\delta)), \boldsymbol{\mu}_{-i}\right) & =(\check{p}-\delta-c) D(\check{p}-\delta) \\
& >\pi_{i}\left((\check{p}, D(\check{p})), \boldsymbol{\mu}_{-i}\right) .
\end{aligned}
$$

This implies that $\mu_{p}$ does not have an atom at $\check{p}$.

Proof of Lemma 4.3.3. First observe that Lemma 4.3.2 implies $\check{p}<p^{m}$, because otherwise $\check{p}=p^{m}=\hat{p}$ and $\mu(\{\check{p}\})>0$. Next, we will show that there exists a price $p^{\prime} \in\left(\check{p}, p^{m}\right]$ for which $s\left(p^{\prime}\right)=\left\{D\left(p^{\prime}\right)\right\}$. In the proof of Lemma 4.3.2, it is shown that for any $p \in\left[\check{p}, p^{m}\right]$, it holds for all $q>D(p)$ that $q \notin$ $s(p)$. Therefore, given that all $n-1$ rivals stick to their equilibrium strategy, firm $i$ 's profit of choosing $(p, q) \in \operatorname{supp}(\mu)$, is as follows. The expected residual demand of firm $i$, when $m \geq 1$ firms price below, $l$ firms price at the same 
price and $n-m-l-1$ price above the price of firm $i$, is given by

$$
\begin{aligned}
A\left((p, q), \boldsymbol{\mu}_{-\boldsymbol{i}}\right)= & \left(\sum_{m=1}^{n-1} \sum_{l=0}^{n-1-m} \int_{([\check{p}, p) \times[0, D(c)])^{m} \times(\{p\} \times[0, D(c)])^{l}}\right. \\
& \min \left\{q, \frac{q}{\sum_{j \in \Omega(p)} q_{j}}\left(D(p)-\sum_{k \in \Delta(p)} q_{k}\right)^{+}\right\} d\left(\prod_{j=1}^{m+l} \mu\left(p_{j}, q_{j}\right)\right) \\
& \left.\times \frac{(n-1) !}{(n-1-m) ! m !} \times \frac{(n-1-m) !}{(n-1-m-l) ! l !}\right) \times\left(\mu_{p}((p, \hat{p}])\right)^{n-m-l-1} .
\end{aligned}
$$

The expected residual demand of firm $i$, when $l \geq 1$ firms price at the same price and $n-l-1$ firms price above the price of firm $i$, is given by

$$
\begin{aligned}
B\left((p, q), \boldsymbol{\mu}_{-i}\right)= & \left(\sum_{l=1}^{n-1} \int_{\left(\{p\} \times[0, D(c)]^{l}\right.} \min \left\{q, \frac{q}{\sum_{j \in \Omega(p)} q_{j}} D(p)\right\} d\left(\prod_{j=1}^{l} \mu\left(p_{j}, q_{j}\right)\right)\right. \\
& \left.\times \frac{(n-1) !}{(n-1-l) ! l !}\right) \times\left(\mu_{p}((p, \hat{p}])\right)^{n-l-1} .
\end{aligned}
$$

Therefore, firm $i$ 's expected profit of choosing $(p, q) \in \operatorname{supp}(\mu)$ is

$$
\begin{aligned}
\pi_{i}\left((p, q), \boldsymbol{\mu}_{-i}\right)= & p \times A\left((p, q), \boldsymbol{\mu}_{-\boldsymbol{i}}\right)+p \times B\left((p, q), \boldsymbol{\mu}_{-\boldsymbol{i}}\right) \\
& +p q\left(\mu_{p}((p, \hat{p}])\right)^{n-1}-c q .
\end{aligned}
$$

Both $A\left((p, q), \boldsymbol{\mu}_{-\boldsymbol{i}}\right)$ and $B\left((p, q), \boldsymbol{\mu}_{-\boldsymbol{i}}\right)$ are increasing in $q$ for $q \leq D(p)$. Hence, $\pi_{i}\left((p, q), \boldsymbol{\mu}_{-\boldsymbol{i}}\right)$ is strictly increasing in $q$ when $p\left(\mu_{p}((p, \hat{p}])\right)^{n-1}-c>0$.

Since there are at most countably many atoms in $\mu_{p}, \check{p}>c$ and $\mu_{p}(\{\check{p}\})=0$ (Lemma 4.3.2), there exists a $p^{\prime} \in\left(\check{p}, p^{m}\right]$ such that $p\left(\mu_{p}((p, \hat{p}])\right)^{n-1}-c>0$ for all $p \in\left(\check{p}, p^{\prime}\right]$ and $s\left(p^{\prime}\right) \neq \varnothing$. We now show that $s(p)=\{D(p)\}$ or $s(p)=\varnothing$ for all $p \in\left(\check{p}, p^{\prime}\right]$. Assume $p \in\left(\check{p}, p^{\prime}\right], q \in s(p)$ and $q<D(p)$. Define $B_{\varepsilon}(p, q)$ as the open $\varepsilon$-ball centered at $(p, q)$ and let $\varepsilon>0$ be small enough, such that for all $\left(p_{i}, q_{i}\right) \in B_{\varepsilon}(p, q)$ it holds that $q_{i}<D\left(p_{i}\right), p_{i}\left(\mu_{p}\left(\left(p_{i}, \hat{p}\right]\right)\right)^{n-1}-c>0$ and $p_{i}>\check{p}$. Let $(\tilde{p}, \tilde{q})$ be an arbitrary element in $B_{\varepsilon}(p, q)$. Then, since the profit of firm $i$ is increasing in $q$ for $q \leq D(p)$, firm $i$ strictly improves its profits by choosing $(\tilde{p}, D(\tilde{p}))$. Therefore, for any $(\tilde{p}, \tilde{q}) \in B_{\varepsilon}(p, q)$ there is another combination of price and quantity that will give a strictly higher payoff and therefore $q \notin s(p)$. In the proof of Lemma 4.3.2, it is shown that for all $q \in s(p)$ it holds that $q \leq D(p)$. This gives $s(p)=\{D(p)\}$ or $s(p)=\varnothing$ for all $p \in\left(\check{p}, p^{\prime}\right]$. Since $s\left(p^{\prime}\right) \neq \varnothing$, it holds that $s\left(p^{\prime}\right)=\left\{D\left(p^{\prime}\right)\right\}$.

Now suppose that $\mu_{p}$ has an atom at some price $p \in\left(\check{p}, p^{\prime}\right]$. The measure $\mu_{p}$ has at most countably many atoms, which implies that there exists a 
small enough $\delta>0$ such that $p-\delta>\check{p}, \mu_{p}(\{p-\delta\})=0$ and

$$
\begin{aligned}
\pi_{i}\left((p-\delta, D(p-\delta)), \boldsymbol{\mu}_{-i}\right) & \left.=(p-\delta) D(p-\delta) \mu_{p}((p-\delta, \hat{p}])\right)^{n-1}-c D(p-\delta) \\
& \left.\geq(p-\delta) D(p-\delta) \mu_{p}([p, \hat{p}])\right)^{n-1}-c D(p-\delta) \\
& >p D(p)\left(\sum_{l=0}^{n-1} \frac{1}{n-l} \mu_{p}(\{p\})^{n-1-l} \mu((p, \hat{p}])^{l} \times \frac{(n-1) !}{(n-1-l) ! l !}\right)-c D(p) \\
& =\pi_{i}\left((p, D(p)), \boldsymbol{\mu}_{-i}\right) .
\end{aligned}
$$

In the third line, $l$ represents the number of firms that price at the same level as firm $i$. Since there is an atom at $p$, the probability of $l$ being strictly positive is strictly bigger than 0 , hence the inequality before the third line. Consequently, $\mu_{p}$ cannot have an atom at any price $p \in\left(\check{p}, p^{\prime}\right]$.

Finally, we show that $s(p)=\{D(p)\}$ for all $p \in\left(\check{p}, p^{\prime}\right]$. Assume that $s(p)=$ $\varnothing$ for all $p$ in some interval $(\alpha, \beta) \subseteq\left(\check{p}, p^{\prime}\right)$. This implies that, for $p \in(\alpha, \beta)$,

$$
\pi_{i}\left((p, D(p)), \boldsymbol{\mu}_{-i}\right)=p D(p)\left(\mu_{p}([\beta, \hat{p}])\right)^{n-1}-c D(p) .
$$

It follows from $\beta \leq p^{m}$ and $D^{\prime}(p)(p-c)+D(p)>0$ for all $p \in(\alpha, \beta)$, that $\pi_{i}\left((p, D(p)), \boldsymbol{\mu}_{-i}\right)$ is strictly increasing for all $p \in(\alpha, \beta)$.

Now, the maximum profit that a firm can make in any point in an open $\varepsilon$ ball centered at $(\alpha, D(\alpha))$ is strictly smaller than $(\alpha+\varepsilon) D(\alpha+\varepsilon) \mu_{p}((\alpha-\varepsilon, \check{p}])-$ $c D(\alpha+\varepsilon)$. Since $\pi_{i}\left((p, D(p)), \boldsymbol{\mu}_{-\boldsymbol{i}}\right)$ is strictly increasing for all $p \in(\alpha, \beta)$ and $\mu_{p}$ does not have an atom at any price $p \in\left(\check{p}, p^{\prime}\right]$, as $\varepsilon \rightarrow 0$, there always exists $\gamma \in(\alpha, \beta)$ such that

$$
\pi_{i}\left((\gamma, D(\gamma)), \boldsymbol{\mu}_{-i}\right)>(\alpha+\varepsilon) D(\alpha+\varepsilon) \mu_{p}((\alpha-\varepsilon, \check{p}])-c D(\alpha+\varepsilon) .
$$

That is, there always exists a price-quantity combination $(\gamma, D(\gamma))$ that gives a strictly higher profit than any point in $B_{\varepsilon}(\alpha, D(\alpha))$. This contradicts $(\alpha, D(\alpha)) \in$ $\operatorname{supp}(\mu)$. Thus, we conclude that $s(p)=\{D(p)\}$ for all $p \in\left(\check{p}, p^{\prime}\right]$.

Proof of Theorem 4.3.4. Suppose $\check{p}>c$ and let $p^{u}=\sup \left\{p^{\prime} \in\left[\check{p}, p^{m}\right] \mid\right.$ $\forall p \in\left[\check{p}, p^{\prime}\right): s(p)=\{D(p)\}$ and $\left.\mu_{p}(\{p\})=0\right\}$. From Lemma 4.3.3 it follows that $p^{u}>\check{p}$. Moreover, since the support of a measure is closed, $D\left(p^{u}\right) \in s\left(p^{u}\right)$.

Since $\mu_{p}(\{p\})=0$ for all $p \in\left[\check{p}, p^{u}\right)$, it follows directly from the proof of Lemma 4.3.3 that $\pi^{*}=D(p)\left[p\left(\mu_{p}((p, \hat{p}])\right)^{n-1}-c\right]$ for all $p \in\left[\check{p}, p^{u}\right)$. As $\pi^{*} \geq 0$, it follows that

$$
\begin{aligned}
p^{u}\left(\mu_{p}\left(\left[p^{u}, \hat{p}\right]\right)\right)^{n-1}-c & =\lim _{p \uparrow p^{u}} p\left(\mu_{p}((p, \hat{p}])\right)^{n-1}-c \\
& \geq 0 .
\end{aligned}
$$


Hence, there are two possibilities. Either $p^{u}\left(\mu_{p}\left(\left[p^{u}, \hat{p}\right]\right)\right)^{n-1}-c=0$ or $p^{u}\left(\mu_{p}\left(\left[p^{u}, \hat{p}\right]\right)\right)^{n-1}-$ $c>0$.

Suppose that $p^{u}\left(\mu_{p}\left(\left[p^{u}, \hat{p}\right]\right)\right)^{n-1}-c=0$. Then it follows from $\mu(\{p\})=0$ for all $p \in\left[\check{p}, p^{u}\right)$ that

$$
\begin{aligned}
\pi^{*} & =\lim _{p \uparrow p^{u}} D(p)\left[p\left(\mu_{p}((p, \hat{p}])\right)^{n-1}-c\right] \\
& =D\left(p^{u}\right)\left[p^{u}\left(\mu_{p}\left(\left[p^{u}, \hat{p}\right]\right)\right)^{n-1}-c\right] \\
& =0 .
\end{aligned}
$$

This implies that $p\left(\mu_{p}((p, \hat{p}])\right)^{n-1}-c=0$ for all $p \in\left[\check{p}, p^{u}\right)$. Since $\mu(\{\check{p}\})=0$, it follows that $\left(\mu_{p}((\check{p}, \hat{p}])\right)^{n-1}=1$ and therefore $\check{p}=c$.

Now suppose that $p^{u}\left(\mu_{p}\left(\left[p^{u}, \hat{p}\right]\right)\right)^{n-1}-c>0$. If this is the case, it must hold that $\mu_{p}\left(\left\{p^{u}\right\}\right)>0$. Suppose the contrary, i.e. $\mu_{p}\left(\left\{p^{u}\right\}\right)=0$. Then there exists a $\tilde{p}>p^{u}$ for which it holds that $\tilde{p}\left(\mu_{p}((\tilde{p}, \hat{p}])\right)^{n-1}-c>0$. From the proof of Lemma 4.3.3 it follows that $s(p)=\{D(p)\}$ and $\mu_{p}(\{p\})=0$ for all $p \in[\check{p}, \tilde{p})$. This contradicts the definition of $p^{u}$. Hence, $\mu_{p}\left(\left\{p^{u}\right\}\right)>0$. Given that $\mu_{p}\left(\left\{p^{u}\right\}\right)>0$, for every $q<D\left(p^{u}\right)$, there exists a $\delta>0$ such that

$$
\begin{aligned}
\pi_{i}\left(\left(p^{u}, q\right), \boldsymbol{\mu}_{-\boldsymbol{i}}\right) & \leq q\left[p^{u}\left(\mu_{p}\left(\left[p^{u}, \hat{p}\right]\right)\right)^{n-1}-c\right] \\
& <D\left(p^{u}-\delta\right)\left[\left(p^{u}-\delta\right)\left(\mu_{p}\left(\left(p^{u}-\delta, \hat{p}\right]\right)\right)^{n-1}-c\right] \\
& =\pi_{i}\left(\left(p^{u}-\delta, D\left(p^{u}-\delta\right)\right), \boldsymbol{\mu}_{-\boldsymbol{i}}\right) \\
& =\pi^{*} .
\end{aligned}
$$

The first inequality follows since firm $i$ sells at most $q$. Since $q<D\left(p^{u}\right)$ and $\lim _{p \uparrow p^{u}} D(p)\left[p\left(\mu_{p}((p, \hat{p}])\right)^{n-1}-c\right]=D\left(p^{u}\right)\left[p^{u}\left(\mu_{p}\left(\left[p^{u}, \hat{p}\right]\right)\right)^{n-1}-c\right]$, the second inequality is strict for a small enough $\delta$.

Now, for $q=D\left(p^{u}\right)$ it holds for all $0<\delta \leq p^{u}-\check{p}$ that

$$
\begin{aligned}
\pi_{i}\left(\left(p^{u}, D\left(p^{u}\right)\right), \boldsymbol{\mu}_{-\boldsymbol{i}}\right) & <D\left(p^{u}\right)\left[p^{u}\left(\mu_{p}\left(\left[p^{u}, \hat{p}\right]\right)\right)^{n-1}-c\right] \\
& =\lim _{p \uparrow p^{u}} D(p)\left[p\left(\mu_{p}((p, \hat{p}])\right)^{n-1}-c\right] \\
& =D\left(p^{u}-\delta\right)\left[\left(p^{u}-\delta\right)\left(\mu_{p}\left(\left(p^{u}-\delta, \hat{p}\right]\right)\right)^{n-1}-c\right] \\
& =\pi^{*} .
\end{aligned}
$$

Since there is an atom at $p^{u}$, firm $i$ expects to sell strictly less than $D\left(p^{u}\right)$ when it sets a price of $p^{u}$. Hence, the first inequality is strict and the equalities follow immediately.

So, given that $\mu_{p}\left(\left\{p^{u}\right\}\right)>0$, for any combination $\left(p^{u}, q\right)$ there exists a $\delta>0$ 
such that it holds that $\left(p^{u}-\delta, D\left(p^{u}-\delta\right)\right.$ ) gives a strictly higher payoff. Therefore, this second situation cannot occur. Thus, we conclude that $\check{p}=c$ and $\pi^{*}=0$.

Proof of Lemma 4.5.1. As we consider a price cartel, firms are free in choosing their production levels. Therefore, it is sufficient to analyze the impact of output decisions on per-period profits. Given a fixed cartel price $p^{c} \in\left(c, p^{m}\right]$, per-period profit for every firm $i \in N$ is

$$
\pi_{i}^{c}\left(p^{c}, \mathbf{q}\right)=p^{c} D_{i}\left(p^{c}, \mathbf{q}\right)-c q_{i},
$$

where firm demand is given by

$$
D_{i}\left(p^{c}, \mathbf{q}\right)= \begin{cases}q_{i} & \text { if } \sum_{j \in N} q_{j} \leq D\left(p^{c}\right) \\ \frac{q_{i}}{\sum_{j \in N} q_{j}} D\left(p^{c}\right) & \text { if } \sum_{j \in N} q_{j}>D\left(p^{c}\right) .\end{cases}
$$

The following first-order condition specifies the impact of a change in the level of individual production on per-period profit.

$$
\frac{d \pi_{i}^{c}\left(p^{c}, \mathbf{q}\right)}{d q_{i}}= \begin{cases}p^{c}-c & \text { if } q_{i} \leq D\left(p^{c}\right)-\sum_{j \in N \backslash\{i\}} q_{j} \\ p^{c} \frac{\sum_{j \in N \backslash i j\}} q_{j}}{\left(\sum_{j \in N} q_{j}\right)^{2}} D\left(p^{c}\right)-c & \text { if } q_{i}>D\left(p^{c}\right)-\sum_{j \in N \backslash\{i\}} q_{j}\end{cases}
$$

Hence, as $p^{c}>c$, the profit of firm $i$ is increasing in its production level as long as $q_{i} \leq D\left(p^{c}\right)-\sum_{j \in N \backslash\{i\}} q_{j}$. Yet, profits may increase even more for output levels that make aggregate supply exceed market demand. This requires

$$
p^{c} \frac{\sum_{j \in N \backslash\{i\}} q_{j}}{\left(\sum_{j \in N} q_{j}\right)^{2}} D\left(p^{c}\right)-c \geq 0
$$

or

$$
q_{i} \leq \sqrt{\frac{p^{c} D\left(p^{c}\right)\left(\sum_{j \in N \backslash\{i\}} q_{j}\right)}{c}}-\sum_{j \in N \backslash\{i\}} q_{j} .
$$

Therefore, this case applies when there exists a production level $q_{i}$ for which the following condition is satisfied

$$
D\left(p^{c}\right)-\sum_{j \in N \backslash\{i\}} q_{j}<q_{i} \leq \sqrt{\frac{p^{c} D\left(p^{c}\right)\left(\sum_{j \in N \backslash\{i\}} q_{j}\right)}{c}}-\sum_{j \in N \backslash\{i\}} q_{j} .
$$


This is possible if and only if

$$
\sum_{j \in N \backslash\{i\}} q_{j}>\frac{D\left(p^{c}\right) c}{p^{c}} .
$$

Thus, we can conclude that $q_{i}^{*}\left(p^{c}, \mathbf{q}_{-\mathbf{i}}\right)=D\left(p^{c}\right)-\sum_{j \in N \backslash\{i\}} q_{j}$ if $\sum_{j \in N \backslash\{i\}} q_{j} \leq$ $\frac{D\left(p^{c}\right) c}{p^{c}}$. Moreover, if $\sum_{j \in N \backslash\{i\}} q_{j}>\frac{D\left(p^{c}\right) c}{p^{c}}$, then $q_{i}^{*}\left(p^{c}, \mathbf{q}_{-\mathbf{i}}\right)=\sqrt{\frac{p^{c} D\left(p^{c}\right)\left(\sum_{j \in N \backslash \backslash i\}} q_{j}\right)}{c}}-$ $\sum_{j \in N \backslash\{i\}} q_{j}$ provided that this yields more profit than with zero production. Producing nothing is strictly preferred when the following two equations hold simultaneously:

$$
D\left(p^{c}\right)-\sum_{j \in N \backslash\{i\}} q_{j}<0,
$$

and

$$
p^{c} \frac{\sum_{j \in N \backslash\{i\}} q_{j}}{\left(\sum_{j \in N} q_{j}\right)^{2}} D\left(p^{c}\right)-c<0 .
$$

If $q_{i}=0$, then $\sum_{j \in N} q_{j}=\sum_{j \in N \backslash\{i\}} q_{j}$. Substituting in (4.11) and rearranging gives

$$
\sum_{j \in N \backslash\{i\}} q_{j}>\frac{D\left(p^{c}\right) p^{c}}{c} .
$$

Notice that (4.12) implies (4.10). Hence, (4.12) is a sufficient condition under which firm $i$ finds it optimal to produce nothing. We therefore conclude that $q_{i}^{*}\left(p^{c}, \mathbf{q}_{-\mathbf{i}}\right)=\sqrt{\frac{p^{c} D\left(p^{c}\right)\left(\sum_{j \in N \backslash\{i\}} q_{j}\right)}{c}}-\sum_{j \in N \backslash\{i\}} q_{j}$ if $\frac{D\left(p^{c}\right) c}{p^{c}}<\sum_{j \in N \backslash\{i\}} q_{j} \leq \frac{D\left(p^{c}\right) p^{c}}{c}$ and $q_{i}^{*}\left(p^{c}, \mathbf{q}_{-\mathbf{i}}\right)=0$ if $\sum_{j \in N \backslash\{i\}} q_{j}>\frac{D\left(p^{c}\right) p^{c}}{c}$.

Proof of Lemma 4.5.2. Let $m=\sum_{k \in N \backslash\{v, w\}} q_{k}$. Hence,

$$
q_{v}^{*}=\sqrt{\frac{p^{c} D\left(p^{c}\right)\left(m+q_{w}^{*}\right)}{c}}-\left(m+q_{w}^{*}\right),
$$

and

$$
q_{w}^{*}=\sqrt{\frac{p^{c} D\left(p^{c}\right)\left(m+q_{v}^{*}\right)}{c}}-\left(m+q_{v}^{*}\right) .
$$

Substituting $q_{w}^{*}$ in $q_{v}^{*}$ yields

$$
4 q_{v}^{* 2}+\left(4 m-\frac{p^{c} D\left(p^{c}\right)}{c}\right) q_{v}^{*}-m\left(\frac{p^{c} D\left(p^{c}\right)}{c}-m\right)=0 .
$$


Solving for $q_{v}^{*}$ gives

$$
q_{v}^{*}=\frac{-\left(4 m-\frac{p^{c} D\left(p^{c}\right)}{c}\right) \pm \sqrt{\frac{p^{c} D\left(p^{c}\right)}{c}\left(8 m+\frac{p^{c} D\left(p^{c}\right)}{c}\right)}}{8} .
$$

There is a unique solution for which $q_{v}^{*}>0$. As we have a similar expression for $q_{w}^{*}$, we conclude $q_{v}^{*}=q_{w}^{*}$.

Proof of Theorem 4.5.3. For a given cartel price $p^{c}$, a cartel contract $\left(p^{c}, \mathbf{q}\right)$ is a subgame perfect Nash equilibrium when none of the firms has an incentive to change its production level given the output of rivals. There are two possibilities. For all $i \in N$, either (i) $\sum_{j \in N \backslash\{i\}} q_{j} \leq D\left(p^{c}\right) c / p^{c}$, or (ii) $D\left(p^{c}\right) c / p^{c}<\sum_{j \in N \backslash\{i\}} q_{j} \leq D\left(p^{c}\right) p^{c} / c$.

(i) By Lemma 4.5.1, it holds that $q_{i}^{*}\left(p^{c}, \mathbf{q}_{-\mathbf{i}}\right)=D\left(p^{c}\right)-\sum_{j \in N \backslash\{i\}} q_{j}$ for all $i \in N$. As $D\left(p^{c}\right)-\sum_{j \in N \backslash\{i\}} q_{j} \geq D\left(p^{c}\right)-\frac{D\left(p^{c}\right) c}{p^{c}}$, it follows that $q_{i}^{*}\left(p^{c}, \mathbf{q}_{-\mathbf{i}}\right) \geq$ $\frac{p^{c}-c}{p^{c}} D\left(p^{c}\right)$.

In turn, this implies

$$
n \frac{p^{c}-c}{p^{c}} D\left(p^{c}\right) \leq D\left(p^{c}\right) .
$$

Rearranging gives

$$
p^{c} \leq \frac{c n}{n-1} .
$$

(ii) By Lemma 4.5.1, it holds that $q_{i}^{*}\left(p^{c}, \mathbf{q}_{-\mathbf{i}}\right)=\sqrt{\frac{p^{c} D\left(p^{c}\right)\left(\sum_{j \in N \backslash i\}} q_{j}\right)}{c}}-\sum_{j \in N \backslash\{i\}} q_{j}$ for all $i \in N$. In this case, we know by Lemma 4.5.2 that all firms produce the same quantity. Therefore,

$q_{i}^{*}\left(p^{c}, \mathbf{q}_{-\mathbf{i}}\right)=\sqrt{\frac{p^{c} D\left(p^{c}\right)\left(\sum_{j \in N \backslash\{i\}} q_{j}\right)}{c}}-\sum_{j \in N \backslash\{i\}} q_{j}=\sqrt{\frac{p^{c} D\left(p^{c}\right)(n-1) q_{i}^{*}\left(p^{c}, \mathbf{q}_{-\mathbf{i}}\right)}{c}}-(n-1) q_{i}^{*}\left(p^{c}, \mathbf{q}_{-\mathbf{i}}\right)$.

Rearranging gives

$$
q_{i}^{*}\left(p^{c}, \mathbf{q}_{-\mathbf{i}}\right)=\frac{p^{c} D\left(p^{c}\right)(n-1)}{c n^{2}} .
$$

As $D\left(p^{c}\right) c / p^{c}<\sum_{j \in N \backslash\{i\}} q_{j}$, it follows that $(n-1) q_{i}^{*}=\frac{p^{c}(n-1)^{2} D\left(p^{c}\right)}{c n^{2}}>D\left(p^{c}\right) c / p^{c}$.

Rearranging gives

$$
p^{c}>\frac{c n}{n-1} .
$$

Finally, it should also hold that $(n-1) q_{i}^{*} \leq D\left(p^{c}\right) p^{c} / c$, which is true as $\frac{(n-1)^{2}}{n^{2}}<$ 1. 
Proof of Theorem 4.5.6. If $p^{m} \leq \frac{c n}{n-1}$, then $p^{c} \leq \frac{c n}{n-1}$. By Theorem 4.5.3, $q_{i}^{*}=D\left(p^{c}\right)-\sum_{j \in N / i} q_{j}^{*}$. In this case, the incentive compatibility constraint is given by

$$
\delta \geq \delta_{i}^{*}=1-\frac{q_{i}^{*}}{\sum_{j \in N} q_{j}^{*}}, \text { for all } i \in N .
$$

Hence, the critical discount factor is highest for the firm(s) with the lowest level of sales. Clearly, if $\delta \geq \delta_{i}^{*}$ for all $i \in N$, then there exists both a viable price cartel and a price-quota cartel. If $1-\frac{1}{n} \leq \delta<1-\frac{q_{i}^{*}}{\sum_{j \in N} q_{j}^{*}}$ for some firm $i \in N$, then a price cartel is not viable. In this case, the cartel is only viable when a sufficiently large share of market demand is allocated to the smallest cartel member(s). There exists an allocation of sales for which the pricequota cartel is viable, because $\delta \geq 1-\frac{1}{n}$.

Proof of Theorem 4.5.7. If $p^{m}>\frac{c n}{n-1}$, then $p^{c}<p^{m}$ and $q_{i}^{*}=\frac{(n-1) p^{c} D\left(p^{c}\right)}{c n^{2}}$ for all $i \in N$. Moreover, $\sum_{i \in N} q_{i}^{*} \geq D\left(p^{c}\right)$. The collusive value of every member of a price cartel is then given by $V_{i}\left(p^{c}, \mathbf{q}^{*}\right)=\frac{\frac{q_{i}^{*}}{\sum_{i \in N} q_{i}^{*}}\left(p^{c} D\left(p^{c}\right)-c \sum_{i \in N} q_{i}^{*}\right)}{1-\delta}$. Clearly, keeping the cartel price and market shares fixed, eliminating all excess supply increases the collusive value of every member. This would yield a situation in which all firms produce $\frac{D\left(p^{c}\right)}{n}$ and the market clears. In this case, firms optimally set the monopoly price, which is sustainable as $\delta \geq 1-\frac{1}{n}$ by assumption.

Proof of Theorem 4.6.1. See proof of Theorem 4.5.6. 



\section{Bibliography}

B. Allen. "Price and Quantity Competition in Homogeneous Markets". Economic Letters, 38:417-422, 1992.

B. Allen and M. Hellwig. "Bertrand-Edgeworth Duopoly with Proportional Residual Demand”. International Economic Review, 34:39-60, 1993.

Y. Amihud and H. Mendelson. "Price Smoothing and Inventory". Review of Economic Studies, 50:87-98, 1983.

S. Athey and K. Bagwell. "Optimal Collusion with Private Information". RAND Journal of Economics, 32:428-465, 2001.

S. Athey, K. Bagwell, and C. Sanchirico. "Collusion and Price Rigidity". The Review of Economic Studies, 71:317-349, 2004.

J. Benoit and V. Krishna. "Dynamic Duopoly: Prices and Quantities". The Review of Economic Studies, 54:23-35, 1987.

D. Besanko and U. Doraszelski. "Capacity Dynamics and Endogenous Asymmetries in Firm Size”. RAND Journal of Economics, 35:23-49, 2004.

G. Biglaiser and N. Vettas. "Dynamic Price Competition with Capacity Constraints and Strategic Buyers". CEPR Discussion Paper No. 4315, 2004.

S. Bikhchandani and J. Mamer. "A Duopoly Model of Pricing for Inventory Liquidation". European Journal of Operational Research, 69:177-186, 1993. 
I. Bos and J. Harrington. "Endogenous Cartel Formation with Heterogeneous Firms". RAND Journal of Economics, 41:92-117, 2010.

T. Bresnahan and P. Reiss. "Dealer and Manufacturer Margins". RAND Journal of Economics, 16:252-268, 1985.

X. Chen and D. Simchi-Levi. "Coordinating Inventory Control and Pricing Strategies: The Continuous Review Model". Operations Research Letters, 34:323-332, 2006.

E. Choi, C. Menezes, and J. Tressler. "A Theory of Price-Fixing Rings". Quarterly Journal of Economics, 100:465-478, 1985.

J. Connor. "Our Customers are our Enemies: the Lysine Cartel of 19921995”. Review of Industrial Organization, 18:5-21, 2001.

P. Dasgupta and E. Maskin. The Existence of Equilibrium in Discontinuous Economic Games, I: Theory. The Review of Economic Studies, 53(1):1-26, 1986.

C. Davidson and R. Deneckere. "Excess Capacity and Collusion". International Economic Review, 31:521-541, 1990.

H. Dixon. "The Competitive Outcome as the Equilibrium in an Edgeworthian Price-Quantity Model”. Economic Journal, 102:301-309, 1992.

W. Elmaghraby and P. Kescinocak. "Dynamic Pricing in the Presence of Inventory Considerations: Research Overview, Current Practices, and Future Directions”. Management Science, 49:1297-1309, 2003.

C. Fershtman and E. Muller. "Capital Investments and Price Agreements in Semicollusive Markets”. RAND Journal of Economics, 17:214-226, 1986.

J. Friedman. "On the Strategic Importance of Prices versus Quantities". RAND Journal of Economics, 19:607-622, 1988.

J. Gabszewicz and S. Poddar. "Demand Fluctuations and Capacity Utilization under Duopoly”. Economic Theory, 10:131-146, 1997.

G. Gaudet and N. Van Long. "On the Effects of the Distribution of Initial Endowments in a Nonrenewable Resource Duopoly". Journal of Economic Dynamics and Control, 18:1189-1198, 1994. 
Bibliography

E. Green and R. Porter. "Noncooperative Collusion under Imperfect Price Information”. Econometrica, 52:87-100, 1984.

M. Greenhut and H. Ohta. "Vertical Integration of Successive Oligopolists ". The American Economic Review, 69:137-141, 1979.

J. Harrington. "How Do Cartels Operate?". Foundations and Trends in Microeconomics, 2(1):1-105, 2006.

J. Harrington. "The Determination of Price and Output Quotas in a Heterogeneous Cartel”. International Economic Review, 32:767-792, 1991.

J. Harrington and A. Skrzypacz. "Collusion under Monitoring of Sales". RAND Journal of Economics, 38:314-331, 2007.

J. Harrington and A. Skrzypacz. "Private Monitoring and Communication in Cartels". Forthcoming in American Economic Review, 2010.

H. Hotelling. "The Economics of Exhaustible Resources". Journal of Political Economy, 39:1158-1167, 1931.

J. Hörner and J. Jamison. "Collusion with (Almost) No Information". RAND Journal of Economics, 38:804-822, 2007.

D. Kovenock and S. Roy. "Dynamic Capacity Choice in a BertrandEdgeworth Framework". Journal of Mathematical Economics, 29:135$160,1998$.

D.M. Kreps and J.A. Scheinkman. "Quantity Precommitment and Bertrand Competition Yield Cournot Outcomes". The Bell Journal of Economics, 14 (2):326-337, 1983.

J. Laye and M. Laye. "Uniqueness and Characterization of Capacity Constrained Cournot-Nash Equilibrium". Operations Research Letters, 36: 168-172, 2008.

M.C. Levenstein and V.Y. Suslow. Studies of Cartel Stability: A Comparison of Methodological Approaches. In P.Z. Grossman, editor, How Cartels Endure and How they Fail. Cheltenham, U.K.: Edward Elgar Publishing, 2004.

R. Levitan and M. Shubik. "Price Duopoly and Capacity Constraints". International Economic Review, 13:111-121, 1972. 
T. Lewis and R. Schmalensee. "On Oligopolistic Markets for Nonrenewable Natural Resources". The Quarterly Journal of Economics, 95:475-491, 1980 .

G. Loury. "A Theory of 'Oil'igopoly: Cournot Equilibrium in Exhaustible Resource Markets with Fixed Supplies". International Economic Review, 27:285-301, 1986.

E. Maskin. "The Existence of Equilibrium with Price-Setting Firms". American Economic Review, 76(3):382-386, 1986.

D. Osborne. "Cartel Problems". American Economic Review, 66(5):835-844, 1976.

M. Osborne and C. Pitchik. "Price Competition in a Capacity-Constraint Duopoly". Journal of Economic Theory, 38:238-260, 1986.

D. Pal. "Cournot Duopoly with Two Production Periods and Cost Differentials”. Journal of Economic Theory, 55:441-448, 1991.

D. Pal. "Endogenous Stackelberg Equilibria with Identical Firms". Games and Economic Behavior, 12:81-94, 1996.

C.-Z. Qin and C. Stuart. "Bertrand versus Cournot Revisited". Economic Theory, 10:497-507, 1997.

J. Reinganum and N. Stokey. "Oligopoly Extraction of a Common Property Natural Resource: The Importance of the Period of Commitment in Dynamic Games". International Economic Review, 26(1):161-173, 1985.

P. Reny. "On the Existence of Pure and Mixed Strategy Nash Equilibria in Discontinuous Games”. Econometrica, 67:1029-1056, 1999.

N. De Roos. "Examining Models of Collusion: the Market for Lysine". International Journal of Industrial Organization, 24:1083-1107, 2006.

R. Russo, M.P. Schinkel, A. Günster, and M. Carree. "European Commission Decisions on Competition: Economic Perspectives on Landmark Antitrust and Merger Cases”. New York: Cambridge University Press, 2010.

S. Salo and O. Tahvonen. "Oligopoly Equilibria in Nonrenewable Resource Markets". Journal of Economic Dynamics and Control, 25:671-702, 2001. 
Bibliography

G. Saloner. "Cournot Duopoly with Two Production Periods". Journal of Economic Theory, 42:183-187, 1987.

F. Steen and L. Sørgard. "Semicollusion". Foundations and Trends in Microeconomics, 5:153-228, 2009.

A. Tasnádi. "Production in Advance versus Production to Order". Journal of Economic Behavior, 54:191-204, 2004.

A. Tasnádi. "Price vs. Quantity in Oligopoly Games". International Journal of Industrial Organization, 24:541-554, 2006.

S. Transchel and S. Minner. "The Impact of Dynamic Pricing on the Economic Order Decision”. European Journal of Operational Research, 198: 773-789, 2009.

A. van den Berg and I. Bos. "Collusion in a Price-Quantity Oligopoly". $M E$ TEOR Research Memoranda RM/11/039, 2011.

A. van den Berg, I. Bos, P. Herings, and H. Peters. "Dynamic Cournot Duopoly with Intertemporal Capacity Constraints". Forthcoming in International Journal of Industrial Organization, 2011a.

A. van den Berg, P. Herings, and H. Peters. "The Impact of Bulk-Supply on a Dealer's Sales Strategy". Forthcoming, 2011b.

M. Waterson. "Vertical Integration, Variable Proportions and Oligopoly". The Economic Journal, 92:129-144, 1982.

F. Wirl. "Dynamic Demand and Noncompetitive Intertemporal Output Adjustments". International Journal of Industrial Organization, 28(3):220 229,2010 . 



\section{Nederlandse Samenvatting}

Dat de schaarste van een product of dienst van invloed is op de marktprijs van dat specifieke goed, kan als algemeen bekend verondersteld worden. De beschikbaarheid van een goed is van verschillende factoren afhankelijk. Ten eerste zijn de grondstoffen die gebruikt worden om het goed te produceren en de beschikbaarheid van deze grondstoffen van belang. Daarnaast is er de tijd die het kost om van deze grondstoffen het uiteindelijke product te maken. Dit wordt onder andere beïnvloed door de benodigde arbeid, de stappen in het productieproces en de benodigde machines en kapitaal voor bewerking. De laatste factor van invloed is het aantal schakels in het leveringsproces voordat het product binnen handbereik van de consument is.

In dit proefschrift worden de strategische keuzes van de bedrijven in de laatste schakel geanalyseerd, de bedrijven die het product rechtstreeks aan de consument leveren. Deze bedrijven zijn in verschillende typen markten en sectoren actief. De overeenkomst tussen de bedrijven is dat ze allemaal beperkt zijn in de hoeveelheid goederen die ter plekke kunnen worden geleverd in een bepaalde periode. Daarnaast moeten productie/inkoop beslissingen van te voren worden genomen. Alle bedrijven hebben als doel hun winst te maximaliseren. Wanneer bedrijven met elkaar in concurrentie zijn, is de winst van een bedrijf mede afhankelijk van de strategieën van concurrenten. In dit geval tracht elk bedrijf zijn winst te maximimaliseren, gegéven de strategieën van zijn concurrenten. Als tegelijkertijd voor alle bedrijven geldt dat ze een strategie hanteren die - gegeven de strategieën van concurrenten - de winst maximaliseert, spreken we van een evenwicht. De technieken die worden gebruikt om het gedrag van de bedrijven te analyseren, zijn onder andere afkomstig uit de speltheorie en de systeemtheorie. 
In Hoofdstuk 2 analyseren we het strategisch gedrag van twee bedrijven, die hetzelfde (homogene) goed verkopen. De inkoop/productie beslissing is al voor aanvang van het spel genomen en kan dus niet meer worden aangepast. De bedrijven concurreren een beperkt aantal perioden met elkaar via de verkoop van dit product - in onze analyse is dit twee perioden - waarna de vraag naar het product acuut stopt. Elke periode maken de bedrijven de keuze hoeveel van hun voorraad ze gaan verkopen, wetende dat alles wat ze nu verkopen niet meer tot hun beschikking staat in de volgende periode. Een voorbeeld van dit type concurrentie kan worden gevonden bij handelaren in toegangskaarten voor een (uitverkochte) wedstrijd of concert. Nadat de wedstrijd of het concert heeft plaatsgevonden, zijn de toegangskaarten waardeloos. Tot die tijd kan de handelaar elke dag beslissen hoeveel van de toegangskaarten hij verkoopt en tegen welke prijs. Hierbij kan de handelaar zijn concurrentie niet uit het oog verliezen. Het aanbod van de concurrent bepaalt mede de vraag voor zijn toegangskaarten, nu en in de toekomst. Andere voorbeelden zijn de verkoop van zitplaatsen op een vlucht, hotelkamers, limited edition goederen en modieuze goederen.

Zoals reeds genoemd in het voorbeeld, dient het bedrijf bij het nemen van zijn verkoopbeslissing niet alleen rekening te houden met hoe dit zijn toekomstige voorraad en verkoopmogelijkheden beïnvloedt, maar ook met de verkoopbeslissing van zijn concurrent en de invloed van beide beslissingen op toekomstige verkoop. Het bedrijf kan zich committeren aan zijn verkoopbeslissing, dat wil zeggen, het bepaalt voor aanvang van het spel welk deel van de voorraad het in elke specifieke periode gaat verkopen en wijkt daar niet vanaf. Of het legt zich niet vast en laat elke periode de verkoopbeslissing afhangen van zijn resterende voorraad en van wat het inmiddels heeft geleerd over de strategie van de concurrent. Beide situaties analyseren we, de situatie waarin beide bedrijven committeren en de situatie waarin geen van beiden dat doet. Wanneer beide bedrijven zich committeren aan hun verkoopstrategie blijkt dat er altijd een uniek zuiver evenwicht bestaat waarvoor geldt dat de prijs voor het product stijgt in de tijd. Daarnaast blijkt dat, wanneer het verschil in beginvoorraad tussen de twee bedrijven toeneemt, het consumenten surplus en het totale surplus afnemen. In de tweede situatie, wanneer beide bedrijven zich niet committeren aan hun verkoopbeslissing, bestaat er niet altijd een zuiver evenwicht. Als er in deze situatie wel een zuiver evenwicht bestaat, dan hangt het van 
de beginvoorraden van beide bedrijven af of de prijs voor het product stijgt of daalt in de tijd.

In Hoofdstuk 3 analyseren we de situatie van een monopolist die zijn winst, verkregen uit de verkoop van een product, over de tijd wil maximaliseren. De monopolist koopt zijn voorraden (of een belangrijk onderdeel noodzakelijk voor de productie van het goed) in bij een groothandel, die het product of onderdeel alleen in grote partijen verkoopt. De monopolist bepaalt zelf wanneer hij bijbestelt en welke prijs hij vraagt voor het product. De prijs van het goed kan elk moment worden aangepast. Voorbeelden van dit type monopolie zijn te vinden bij bedrijven die specialistische goederen verkopen. Daarbij kan onder andere worden gedacht aan een webwinkel die cd's verkoopt met daarop niet-commerciële muziek.

We nemen aan dat het bedrijf zo vaak kan bij bestellen als het wil en een vaste prijs betaalt voor het bij te bestellen product. De vraag van de consument naar het product blijft constant over de tijd. Onderzocht is wat het beste bestelmoment is en hoe de monopolist zijn winst tussen bestelmomenten maximaliseert. We vinden dat de monopolist altijd na een vaste tijd bij bestelt. De nieuwe voorraad arriveert zodra de oude voorraad op is. Meteen na het arriveren van de nieuwe voorraad is de prijs van het product het laagst, ergo, het bedrijf verkoopt het meest. De prijs van het product neemt continu toe over de tijd. Dit verkooppatroon herhaalt zich telkens tussen twee opeenvolgende bestelmomenten. Wanneer toekomstige winsten minder waardevol worden zal in sommige gevallen, in tegenstelling tot wat men misschien verwacht, de tijd tussen twee opeenvolgende bestelmomenten toenemen.

In het laatste hoofdstuk, Hoofdstuk 4, analyseren we het strategische gedrag van een aantal bedrijven dat concurreert over de verkoop van hetzelfde product. Iedere periode beslist elk bedrijf hoeveel het inkoopt/ produceert van het goed en welke prijs het vraagt voor de verkoop van het betreffende goed. Het goed is beperkt houdbaar, dat wil zeggen, een overgebleven voorraad kan niet mee worden genomen naar de volgende periode. We bekijken twee mogelijke situaties, één waar de bedrijven hun inkoop/productie beslissing nemen vóórdat en één waarin bedrijven pas produceren/inkopen nádat hun indivuele vraag bekend is. Voorbeelden van dit specifieke type concurrentie kunnen worden gevonden in de voedselindustrie, maar zijn ook van toepassing op andere industrieën. 
We vinden dat in concurrentie, ongeacht de productie situatie, alle bedrijven een verwachte winst van nul hebben. Om de winst te verhogen, kunnen de bedrijven besluiten afspraken te maken over prijs en/of productie/verkoop hoeveelheden. Doorgaans zijn deze afspraken echter illegaal. Een prijs-hoeveelheidsafspraak biedt meer mogelijkheden voor collusie dan een prijsafspraak alleen, maar vereist ook meer coördinatie tussen bedrijven en daarbij de kans om betrapt te worden. Daarom zullen bedrijven alleen bereid zijn om naast een afspraak op prijs ook een hoeveelheidsafspraak te maken, als dit een strict hogere verwachte winst oplevert. In het geval dat de bedrijven van te voren hun productie/verkoop beslissing moeten nemen, vinden we de volgende resultaten. Wanneer de vraag relatief elastisch is, is een prijsafspraak alleen niet altijd mogelijk. Om coördinatie en intentie problemen op te lossen, kan een extra afspraak op hoeveelheid noodzakelijk zijn. Wanneer de consumentenvraag relatief inelastisch is, is een hoeveelheidsafspraak niet nodig om coördinatie en intentie problemen te verhelpen. De enige redenen om, naast prijs, een afspraak op hoeveelheid te maken is de mogelijkheid om een hogere prijs te kunnen vragen en tegelijkertijd overproductie te voorkomen. Wanneer bedrijven pas hun inkoop/productie beslissing hoeven te nemen nadat hun individuele vraag bekend is, blijkt dat soms een afspraak op hoeveelheid, naast een prijsafspraak, noodzakelijk is om samenwerking mogelijk te maken. 


\section{Curriculum Vitae}

Anita van den Berg was born in Boxtel on June 29, 1982. She attended Jacob Roelandlyceum in Boxtel, where she obtained her VWO degree in 2000. Consecutively, she started to study Knowledge Engineering at Maastricht University. In 2004, she became a full-time extra curriculum student in Psychology, Maastricht University. She completed her first studies and obtained her Master's degree in Knowledge Engineering in 2005, with a specialization in the track Operations Research. In 2005, she started the master Econometrics and Operations Research at Maastricht University, and obtained her second Master's degree cum laude in 2006. That same academic year, she also worked at the Department of Knowledge Engineering as project leader "Duale Master". At the end of 2006, Anita became a Ph.D. candidate at the Department of Quantitative Economics, Maastricht University. She spend 5 months at the Kellogg School of Management, Northwestern University (Evanston, USA) as a visiting scholar. Currently, she is employed as a consultant financial risk management at Capgemini Consulting. 